\title{
Pentadecaibins I-V: 15-Residue Peptaibols Produced by a Marine-Derived Trichoderma sp. of the Harzianum Clade
}

Anne-Isaline van Bohemen, ${ }^{\dagger}$ Nicolas Ruiz, ${ }^{*}, \dagger$ Aurore Zalouk-Vergnoux ${ }^{\dagger}$ Aurore Michaud, ${ }^{\dagger}$ Thibaut Robiou du Pont,${ }^{\dagger}$ Irina Druzhinina,,${ }^{\ddagger}$, Lea Atanasova, ${ }^{\perp}$ Soizic Prado, $\|$ Bernard Bodo, $\|$ Laurence Meslet-Cladiere ${ }^{\nabla}$, Bastien Cochereau, ${ }^{\nabla, \dagger}$ Franck Bastide, ${ }^{\#}$ Corentin Maslard, ${ }^{\#}$ Muriel

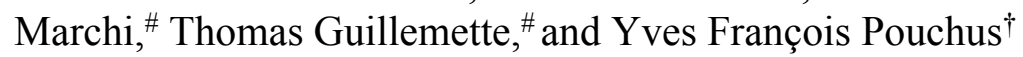

†Université de Nantes, MMS - EA2160, 44000 Nantes, France

$\$$ Institute of Chemical, Environmental and Bioscience Engineering, TU Wien, Vienna, Austria

$\S$ Fungal Genomics Laboratory (FungiG), Nanjing Agricultural University, Nanjing, China

${ }^{\perp}$ Department of Food Science and Technology, University of Natural Resources and Life Sciences - BOKU, Vienna, Austria

\| Muséum National d'Histoire Naturelle, Unité Molécules de Communication et Adaptation des Microorganismes, UMR 7245, CP 54, 57 rue Cuvier, 75005 Paris, France

$\nabla^{\nabla}$ Université de Brest, Laboratoire Universitaire de Biodiversité et Ecologie Microbienne, 29280 Plouzané, France

\# IRHS-UMR1345, Université d'Angers, INRAE, Institut Agro, SFR 4207 QuaSaV, 49071, Beaucouzé, France 


\section{Table of contents}

1/ Morphological analysis of Trichoderma sp. MMS1255

2/ Genomic analyses and phylogeny

3/ ESI-MS profiling of peptaibol-enriched fraction of Trichoderma sp. MMS1255

4/ ESI-MS 2 fragmentation of pentadecaibins I-V (1-5)

5/ Spectral data of pentadecaibins I-V (1-5)

6/ Marfey's analyses of hydrolyzed pentadecaibins I-V (1-5)

7/ Comparison of 15- and 18-residue AA patterns 
1/ Morphological analysis of Trichoderma sp. MMS1255

Fig. S1. Morphological analysis of Trichoderma sp. MMS1255. (a) One week old culture grown on DCA $\left(27^{\circ} \mathrm{C}\right)$. (b) and (c) Conidiophores, phialides and conidia, Congo red staining.
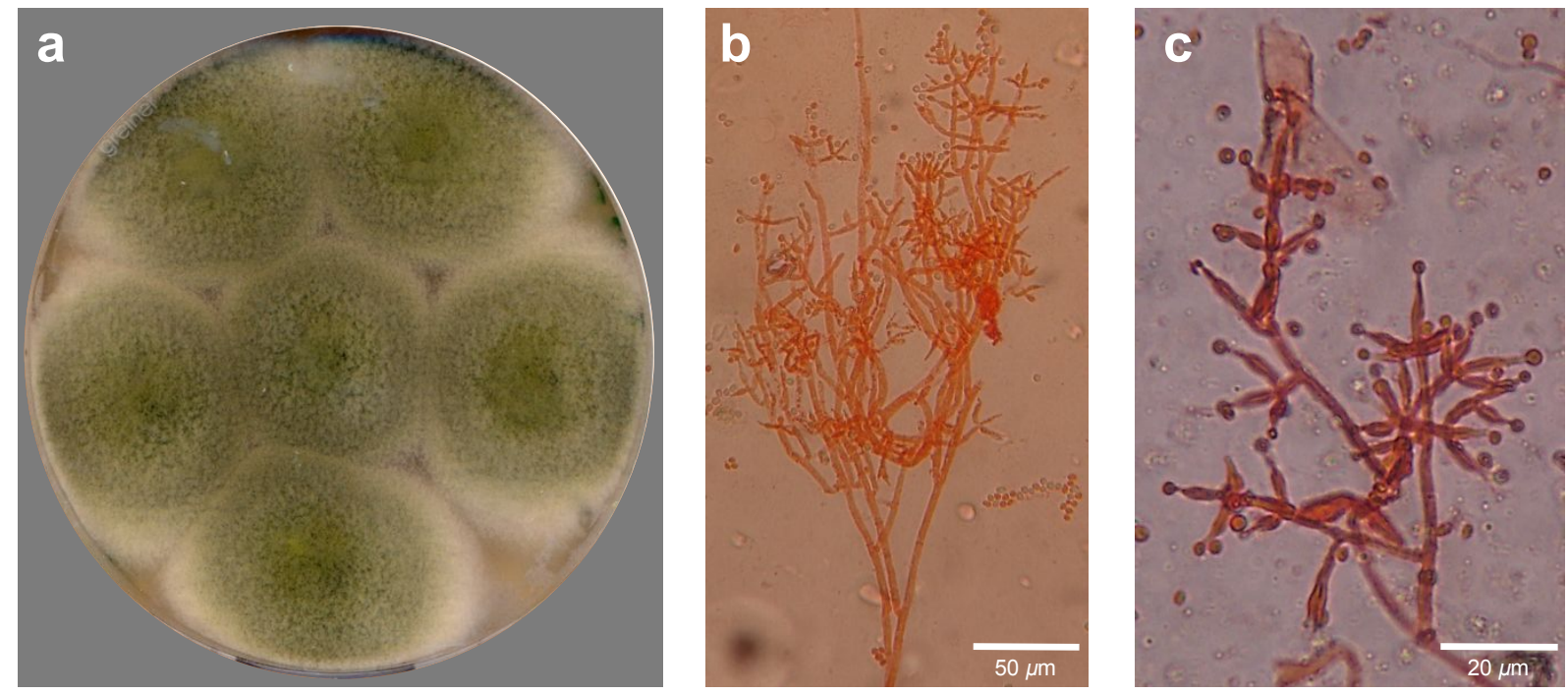
2/ Genomic analyses and phylogeny

Table S1. List of strains and reference sequences used for all phylogenetic analyses 


\begin{tabular}{|c|c|c|c|c|c|c|c|}
\hline \multirow[b]{2}{*}{ Species } & \multirow[b]{2}{*}{ Clade } & \multirow[b]{2}{*}{ Strain no. } & Geographic origin & & GenBank & essior & \\
\hline & & & & tef1 & $r p b 2$ & act & cal \\
\hline T. pyramidale & Green/harzianum & S73 = CBS 135574 & Italy & KJ665699 & KJ665334 & - & - \\
\hline T. guizhouense & Green/harzianum & $\mathrm{S} 628$ & Greece & KJ665511 & KJ665273 & - & - \\
\hline T. simmonsii & Green/harzianum & S7 & Italy & KJ665719 & KJ665337 & - & - \\
\hline T. priscilae & Green/harzianum & S129 & Italy & KJ665689 & KJ665332 & - & - \\
\hline T. priscilae & Green/harzianum & S168 = CBS 131487 & Spain & KJ665691 & KJ665333 & - & - \\
\hline T. christiani & Green/harzianum & S93 & Italy & KJ665442 & KJ665245 & - & - \\
\hline T. christiani & Green/harzianum & CBS $132572=$ S442 & Spain & KJ665439 & KJ665244 & - & - \\
\hline T. sp. S138 & Green/harzianum & $\mathrm{S} 138$ & Italy & KJ665730 & KJ665343 & - & - \\
\hline T. corneum & Green/harzianum & G.J.S. 97-82 & Thailand & KJ665455 & KJ665252 & - & - \\
\hline T. italicum & Green/harzianum & S15 & Italy & KJ665526 & KJ665283 & - & - \\
\hline T. italicum & Green/harzianum & S131 = CBS 132567 & Italy & KJ665525 & KJ665282 & - & - \\
\hline T. hausknechtii & Green/harzianum & Hypo 649 = CBS 133493 & France & KJ665515 & KJ665276 & - & - \\
\hline$T$. sp. S222 & Green/harzianum & $\mathrm{S} 222$ & Spain & KJ665732 & KJ665345 & - & - \\
\hline T. sp. S404 & Green/harzianum & S404 & Spain & KJ665733 & KJ665346 & - & - \\
\hline T. tomentosum & Green/harzianum & S23 & Italy & KJ665759 & KJ665351 & - & - \\
\hline T. helicolixii & Green/harzianum & S640 = CBS 133499 & Greece & KJ665517 & KJ665278 & - & - \\
\hline T. helicolixii & Green/harzianum & S515 = CBS 135583 & Spain & KJ665516 & KJ665277 & - & - \\
\hline T. gliocladium & Green & S81 = CBS 130009 & Italy & KJ665502 & KJ665271 & - & - \\
\hline T. gliocladium & Green & S83 & Italy & KJ665503 & KJ665272 & - & - \\
\hline T. ceramicum & Green & S353 & France & KJ665445 & KJ665248 & - & - \\
\hline T. ceramicum & Green & S366 & France & KJ665446 & KJ665249 & - & - \\
\hline T. longipile & Green & $\mathrm{S} 40$ & Italy & KJ665556 & KJ665292 & - & - \\
\hline T. sp. S169 & Green & S169 & Spain & KJ665731 & KJ665344 & - & - \\
\hline T. spirale & Green & S212 & Spain & KJ665740 & KJ665348 & - & - \\
\hline T. cf. rossicum & Stromaticum & S334 & Spain & KJ665700 & KJ665335 & - & - \\
\hline T. sp. S624 & Green & S624 & Greece & KJ665738 & KJ665347 & - & - \\
\hline T. sinuosum 3 & Green & S270 & Croatia & KJ665723 & KJ665339 & - & - \\
\hline T. sinuosum 4 & Green & S378 & Spain & KJ665729 & KJ665342 & - & - \\
\hline T. sinuosum & Green & S349 & Spain & KJ665728 & KJ665341 & - & - \\
\hline T. sinuosum 2 & Green & S158 & Italy & KJ665722 & KJ665338 & - & - \\
\hline T. sinuosum 2 & Green & S276 & Croatia & KJ665725 & KJ665340 & - & - \\
\hline T. danicum & Green & S553 & Spain & KJ665465 & KJ665255 & - & - \\
\hline T. cremeoides & Green & $\mathrm{S} 112$ & Italy & KJ665456 & KF134790 & - & - \\
\hline T. cremeoides & Green & S192 & Spain & KJ665460 & KJ665254 & - & - \\
\hline T. leguminosarum & aff. Longibrachiatum & S391 & Spain & KJ665548 & KJ665287 & - & - \\
\hline T. leguminosarum & aff. Longibrachiatum & S494 = CBS 130014 & Spain & KJ665551 & KJ665288 & - & - \\
\hline T. leguminosarum & aff. Longibrachiatum & $\mathrm{S} 503$ & Spain & KJ665552 & KJ665289 & - & - \\
\hline T. effusum & Longibrachiatum & C.P.K. 254 = DAOM 230007 & India & KJ665473 & KJ665260 & - & - \\
\hline T. euskadiense & Longibrachiatum & S $377=$ CBS 130013 & Spain & KJ665492 & KJ665269 & - & - \\
\hline T. citrinoviride & Longibrachiatum & $\mathrm{S} 20$ & Italy & KJ665449 & KJ665250 & - & - \\
\hline T. citrinoviride & Longibrachiatum & $\mathrm{S} 27$ & Italy & KJ665450 & KJ665251 & - & - \\
\hline T. hunua & Semiorbis & CBS 238.63 & New Zealand & KJ665519 & KJ665279 & - & - \\
\hline T. albolutescens & Basal & S396 = CBS 131489 & Spain & KJ665354 & KJ665240 & - & - \\
\hline T. ceciliae & Lone lineage & S164 = CBS 130010 & Italy & KJ665444 & KJ665247 & - & - \\
\hline T. rubi & Lone lineage & $S 146=$ CBS 127380 & Italy & KJ665704 & KJ665336 & - & - \\
\hline T. polysporum & Polysporum & S103 & Italy & KJ665673 & KJ665328 & - & - \\
\hline T. polysporum & Polysporum & $S 121=\mathrm{CBS} 136463$ & Italy & KJ665674 & KJ665329 & - & - \\
\hline T. polysporum & Polysporum & S258 & Croatia & KJ665677 & KJ665330 & - & - \\
\hline T. polysporum & Polysporum & S458 & Spain & KJ665682 & KJ665331 & - & - \\
\hline T. polysporum & Polysporum & CBS:112265 = G.J.S. 99-222 & New Zealand & KJ665741 & KJ665349 & - & - \\
\hline T. lacuwombatense & Polysporum & CBS 112266 = G.J.S. 99-198 & New Zealand & KJ665547 & KJ665286 & - & - \\
\hline T. europaeum & Polysporum & Hypo 64 & Czech Republic & KJ665476 & KJ665264 & - & - \\
\hline T. europaeum & Polysporum & Нyро 183 & Germany & KJ665474 & KJ665261 & - & - \\
\hline T. europaeum & Polysporum & Hypo 300 & Austria & KJ665475 & KJ665262 & - & - \\
\hline T. europaeum & Polysporum & S37 & Italy & KJ665484 & KJ665266 & - & - \\
\hline T. europaeum & Polysporum & $\mathrm{S} 134$ & Italy & KJ665481 & KJ665265 & - & - \\
\hline T. europaeum & Polysporum & S611 & Greece & KJ665489 & KJ665268 & - & - \\
\hline T. minutisporum & Polysporum & DAOM 167069 = CBS 341.93 & Canada & KJ665612 & KJ665314 & - & - \\
\hline T. minutisporum & Polysporum & CBS 112255 = G.J.S. 90-82 & USA & KJ665618 & KJ665316 & - & - \\
\hline T. minutisporum & Polysporum & DAOM 216516 = G.J.S. 91-94 & USA & KJ665619 & KJ665317 & - & - \\
\hline T. minutisporum & Polysporum & CBS $112253=$ G.J.S. $90-115$ & USA & KJ665617 & KJ665315 & - & - \\
\hline T. mediterraneum & Polysporum & $\mathrm{S} 12$ & Italy & KJ665562 & KJ665293 & - & - \\
\hline T. mediterraneum & Polysporum & $\mathrm{S} 13$ & Italy & KJ665563 & KJ665294 & - & - \\
\hline T. mediterraneum & Polysporum & $\mathrm{S} 29$ & Italy & KJ665578 & KJ665298 & - & - \\
\hline T. mediterraneum & Polysporum & $\mathrm{S} 30$ & Italy & KJ665580 & KJ665300 & - & - \\
\hline T. mediterraneum & Polysporum & S184 & Spain & KJ665567 & KJ665295 & - & - \\
\hline T. mediterraneum & Polysporum & $\mathrm{S} 190$ & Spain & KJ665568 & KJ665296 & - & - \\
\hline T. mediterraneum & Polysporum & S240 & Spain & KJ665571 & KJ665297 & - & - \\
\hline T. mediterraneum & Polysporum & $\mathrm{S} 292$ & Croatia & KJ665579 & KJ665299 & - & - \\
\hline T. mediterraneum & Polysporum & S410 & Spain & KJ665586 & KJ665302 & - & - \\
\hline T. mediterraneum & Polysporum & S481 & Spain & KJ665599 & KJ665306 & - & - \\
\hline T. mediterraneum & Polysporum & S495 & Spain & KJ665600 & KJ665307 & - & - \\
\hline T. mediterraneum & Polysporum & S522 & Spain & KJ665601 & KJ665308 & - & - \\
\hline T. mediterraneum & Polysporum & S523 & Spain & KJ665602 & KJ665309 & - & - \\
\hline T. mediterraneum & Polysporum & S526 & Spain & KJ665604 & KJ665310 & - & - \\
\hline T. mediterraneum & Polysporum & S594 & Greece & KJ665607 & KJ665311 & - & - \\
\hline T. mediterraneum & Polysporum & $\mathrm{S} 600$ & Greece & KJ665609 & KJ665312 & - & - \\
\hline T. mediterraneum & Polysporum & S621 & Greece & KJ665610 & KJ665313 & - & - \\
\hline T. mediterraneum 1 & Polysporum & S347 & Spain & KJ665582 & KJ665301 & - & - \\
\hline T. mediterraneum 1 & Polysporum & S411 & Spain & KJ665587 & KJ665303 & - & - \\
\hline T. mediterraneum 2 & Polysporum & S451 & Spain & KJ665591 & KJ665304 & - & - \\
\hline T. mediterraneum 2 & Polysporum & S454 & Spain & KJ665592 & KJ665305 & - & - \\
\hline
\end{tabular}


Table S1. Continued

\begin{tabular}{|c|c|c|c|c|c|c|c|}
\hline \multirow[b]{2}{*}{ Species } & \multirow[b]{2}{*}{ Clade } & \multirow[b]{2}{*}{ Strain no. } & \multirow[t]{2}{*}{ Geographic origin } & & GenBank & ccession & \\
\hline & & & & tef1 & $r p b 2$ & act & cal \\
\hline T. hamatum & Viride & Нypo 647 & France & KJ665513 & KJ665274 & - & - \\
\hline T. hamatum & Viride & Нypo 648 & France & KJ665514 & KJ665275 & - & - \\
\hline T. pararogersonii & Viride & S301 = CBS 133496 & Croatia & KJ665625 & KJ665320 & - & - \\
\hline T. neokoningii & Viride & G.J.S. $04-216=$ CBS 120070 & Peru & KJ665620 & KJ665318 & - & - \\
\hline T. stilbohypoxyli & Viride & $\mathrm{S} 24$ & Italy & KJ665742 & KJ665350 & - & - \\
\hline T. paratroviride & Viride & S385 = CBS 136489 & Spain & KJ665627 & KJ665321 & - & - \\
\hline T. paratroviride & Viride & S489 & Spain & KJ665628 & KJ665322 & - & - \\
\hline T. koningiopsis & Viride & S359 & France & KJ665546 & KJ665285 & - & - \\
\hline T. petersenii & Viride & S109 & Italy & KJ665631 & KJ665325 & - & - \\
\hline T. petersenii & Viride & S167 & Spain & KJ665632 & KJ665326 & - & - \\
\hline T. petersenii & Viride & S200 & Portugal & KJ665636 & KJ665327 & - & - \\
\hline T. dingleyae & Viride & CBS $119056=$ G.J.S. $02-50$ & New Zealand & KJ665467 & KJ665257 & - & - \\
\hline T. istrianum & Viride & S123 & Italy & KJ665521 & KJ665280 & - & - \\
\hline T. istrianum & Viride & S310 = CBS 130539 & Croatia & KJ665523 & KJ665281 & - & - \\
\hline T. caribbaeum & Viride & CBS $119093=$ G.J.S. 97-3 & Guadeloupe & KJ665443 & KJ665246 & - & - \\
\hline T. dorotheae & Viride & S231 & Spain & KJ665468 & KJ665258 & - & - \\
\hline T. dorotheae & Viride & S444 & Spain & KJ665469 & KJ665259 & - & - \\
\hline T. decipiens & Hypocreanum & S372 & France & KJ665466 & KJ665256 & - & - \\
\hline T. balearicum & Psychrophilum & S402 = CBS 133222 & Spain & KJ665434 & KJ665242 & - & - \\
\hline$P$. farinosa & out group & C.P.K. 2453 & Germany & EU703890 & EU703936 & - & - \\
\hline$P$. farinosa & out group & TFC 97-168 & Estonia & EU703896 & EU703941 & - & - \\
\hline P. farinosa & out group & CBS 121551 = C.P.K. 2429 & Slovenia & EU703889 & EU703935 & - & - \\
\hline T. atrobrunneum & Green/harzianum & S3 & Italy & KJ665376 & KJ665241 & - & - \\
\hline T. afroharzianum & Green/harzianum & GJS 04-186 & Peru & FJ463301 & FJ442691 & - & - \\
\hline T. afroharzianum & Green/harzianum & GJS 04-197 & Peru & FJ463302 & FJ442740 & - & - \\
\hline Trichoderma sp. & Green/harzianum & HV-2014j strain S278 & Croatia & KF134799 & KF134791 & - & - \\
\hline T. lentiforme & Green/harzianum & DIS $173 f$ & Brazil & FJ463347 & FJ442787 & FJ442444 & FJ442291 \\
\hline T. Ientiforme & Green/harzianum & DIS $218 \mathrm{e}$ & Ecuador & FJ463310 & FJ442793 & FJ442449 & FJ442296 \\
\hline T. lentiforme & Green/harzianum & DIS 64a & Ecuador & FJ463358 & FJ442792 & FJ442480 & FJ442322 \\
\hline T. lixii & Green/harzianum & $\begin{array}{l}\text { CBS } 110080=\text { ATCC MYA-2478 = G.J.S. } \\
97-96=\text { BPI } 745654\end{array}$ & Thailand & AF443938 & - & FJ442533 & AF442872 \\
\hline T. neotropicale & Green/harzianum & G.J.S. 11-185 = LA11 & Peru & HQ022771 & - & KP115268 & KP115279 \\
\hline T. neotropicale & Green/harzianum & G.J.S. $11-187=$ T51 & Peru & FJ967825 & - & KP115269 & KP115280 \\
\hline T. endophyticum & Green/harzianum & LA10 & Peru & HQ022776 & - & KP115265 & KP115281 \\
\hline T. Ientiforme & Green/harzianum & DIS 167c & Brazil & FJ463309 & FJ442689 & FJ442564 & FJ442365 \\
\hline T. lentiforme & Green/harzianum & DIS 167e & Brazil & FJ463333 & FJ442764 & FJ442563 & FJ442366 \\
\hline T. lentiforme & Green/harzianum & CBS 130727 = DIS 168a & Brazil & FJ463344 & FJ442682 & FJ442441 & FJ442288 \\
\hline T. Ientiforme & Green/harzianum & CBS130728 = DIS 169c & Brazil & FJ851873 & FJ442707 & FJ442442 & FJ442289 \\
\hline T. Ientiforme & Green/harzianum & DIS $173 f$ & Brazil & FJ463347 & FJ442787 & FJ442444 & FJ442291 \\
\hline T. lentiforme & Green/harzianum & CBS 130731 = DIS 218h & Ecuador & FJ463350 & FJ442733 & FJ442451 & FJ442297 \\
\hline T. Ientiforme & Green/harzianum & CBS 130734 = DIS 233g & Ecuador & FJ851874 & FJ442717 & FJ442459 & FJ442304 \\
\hline T. lentiforme & Green/harzianum & CBS $130735=$ DIS $246 e$ & Ecuador & FJ851883 & FJ442760 & FJ442565 & FJ442367 \\
\hline T. lentiforme & Green/harzianum & DIS 246j & Ecuador & FJ463346 & FJ442695 & FJ442460 & FJ442305 \\
\hline T. lentiforme & Green/harzianum & CBS 130736 = DIS 246k & Ecuador & FJ463353 & FJ442758 & FJ442461 & FJ442306 \\
\hline T. lentiforme & Green/harzianum & CBS $130737=$ DIS 253b & Ecuador & FJ851875 & FJ442756 & FJ442462 & FJ442307 \\
\hline T. Ientiforme & Green/harzianum & CBS 130738 = DIS 264u & Ecuador & FJ463334 & FJ442788 & FJ442464 & FJ442309 \\
\hline T. lentiforme & Green/harzianum & CBS 130744 = DIS 325ai & Ecuador & FJ463359 & FJ442791 & FJ442470 & FJ442314 \\
\hline T. Ientiforme & Green/harzianum & DIS 354a & Ecuador & FJ463339 & FJ442734 & FJ442473 & FJ442317 \\
\hline T. lentiforme & Green/harzianum & DIS 64a & Ecuador & FJ463358 & FJ442792 & FJ442480 & FJ442322 \\
\hline T. lentiforme & Green/harzianum & DIS 64b & Ecuador & FJ463388 & FJ442736 & FJ442481 & FJ442323 \\
\hline T. lentiforme & Green/harzianum & CBS $130725=$ DIS 67b & Ecuador & FJ463393 & FJ442692 & FJ442482 & FJ442324 \\
\hline T. Ientiforme & Green/harzianum & DIS 93d & Peru & FJ851880 & FJ442683 & FJ442556 & FJ442326 \\
\hline T. Ientiforme & Green/harzianum & DIS 94d & Peru & FJ463379 & FJ442749 & FJ442483 & FJ442327 \\
\hline T. Ientiforme & Green/harzianum & CBS 130433 = G.J.S. 00-06 & Mexico & AF443932 & - & FJ442484 & AF442863 \\
\hline T. lentiforme & Green/harzianum & CBS 130434 = G.J.S. 00-08 & Mexico & AF443931 & FJ442743 & FJ442485 & AF442862 \\
\hline T. lentiforme & Green/harzianum & G.J.S. 00-22 & Mexico & AF443930 & FJ442687 & FJ442487 & AF442861 \\
\hline T. Ientiforme & Green/harzianum & LA 30 & Peru & HQ022761 & - & KP115264 & KP115278 \\
\hline T. harzianum & Green/harzianum & CBS $130496=$ G.J.S. 05-107 & Italy & FJ463329 & FJ442708 & FJ442569 & FJ442333 \\
\hline T. harzianum & Green/harzianum & CBS130674 = ATCC 20900 = G.J.S. 99-5 & USA & FJ442236 & - & FJ442539 & FJ442356 \\
\hline T. harzianum & Green/harzianum & CBS 1304452 = G.J.S. $04-71$ & Italy & FJ463396 & FJ442779 & FJ442494 & FJ442369 \\
\hline T. simmonsii & Green/harzianum & CBS 123765 = G.J.S. 90-127 & USA & AF443936 & FJ442798 & FJ442524 & AF442870 \\
\hline T. simmonsii & Green/harzianum & CBS $123799=$ IMI $393966=$ G.J.S. 90-22 & USA & AF443933 & AY391925 & FJ442581 & AF442867 \\
\hline T. simmonsii & Green/harzianum & CBS $130431=$ G.J.S. 91-138 & USA & AF443935 & FJ442757 & FJ442526 & AF442869 \\
\hline T. simmonsii & Green/harzianum & CBS 546.92 = ATCC MYA-2453 = G.J.S. & USA & AF443937 & FJ442710 & FJ442527 & AF442871 \\
\hline T. camerunense & Green/harzianum & CBS 137272 = G.J.S. 99-230 & Cameroon & AF348107 & - & FJ442537 & AF442875 \\
\hline T. camerunense & Green/harzianum & G.J.S. 99-231 & Cameroon & AF348108 & - & FJ442538 & AF442874 \\
\hline T. rifaii & Green/harzianum & CBS $130745=$ DIS $337 f$ & Panama & FJ463321 & FJ442720 & FJ442471 & FJ442315 \\
\hline T. afarasin & Green/harzianum & CBS $130742=$ DIS $314 f$ & Cameroon & FJ463400 & FJ442778 & FJ442468 & FJ442312 \\
\hline T. afarasin & Green/harzianum & DIS 377a & Cameroon & FJ463322 & FJ442799 & FJ442562 & KP115277 \\
\hline T. afarasin & Green/harzianum & CBS 130501 = G.J.S. 06-98 & Cameroon & FJ463327 & - & FJ442519 & FJ442353 \\
\hline T. afarasin & Green/harzianum & $\begin{array}{l}\text { CBS } 130755=\text { IMI } 393967 \text { = G.J.S. 99- } \\
227\end{array}$ & Cameroon & AF348093 & - & FJ442536 & FJ442388 \\
\hline T. endophyticum & Green/harzianum & DIS 217o & Ecuador & FJ463323 & - & FJ442447 & FJ442294 \\
\hline T. endophyticum & Green/harzianum & CBS $130729=$ IMI $395208=$ DIS 217a & Ecuador & FJ463319 & - & FJ442445 & FJ442292 \\
\hline T. endophyticum & Green/harzianum & CBS $130730=$ DIS $217 \mathrm{~h}$ & Ecuador & FJ463314 & FJ442721 & FJ442446 & FJ442293 \\
\hline T. endophyticum & Green/harzianum & DIS 221d & Ecuador & FJ463389 & FJ442794 & FJ442455 & FJ442300 \\
\hline T. endophyticum & Green/harzianum & DIS 221e & Ecuador & FJ463316 & FJ442775 & FJ442456 & FJ442301 \\
\hline T. endophyticum & Green/harzianum & DIS 220k & Ecuador & FJ463328 & FJ442765 & FJ442454 & FJ442299 \\
\hline T. guizhouense & Green/harzianum & G.J.S. 07-18 & Ghana & FJ463390 & - & FJ442521 & FJ442355 \\
\hline T. guizhouense & Green/harzianum & NBRC $30608=$ IFO $30608=$ G.J.S. $97-28$ & Japan & AY937440 & - & FJ442532 & FJ442379 \\
\hline T. guizhouense & Green/harzianum & $\begin{array}{l}\text { ATCC } 90179=\text { IMI } 374787=\text { G.J.S. } 85- \\
119\end{array}$ & Indonesia & AF443941 & FJ442718 & FJ442523 & AF442881 \\
\hline T. aggressivum & Green/harzianum & CBS 100525 & UK & AF348095 & AF545541 & FJ442433 & AF442859 \\
\hline T. aggressivum & Green/harzianum & CBS 433.95 & Northern Ireland & AF348097 & FJ442704 & FJ442436 & FJ442279 \\
\hline
\end{tabular}


Table S1. Continued

\begin{tabular}{|c|c|c|c|c|c|c|c|}
\hline \multirow[b]{2}{*}{ Species } & \multirow[b]{2}{*}{ Clade } & \multirow[b]{2}{*}{ Strain no. } & \multirow[t]{2}{*}{ Geographic origin } & \multicolumn{4}{|c|}{ GenBank accession } \\
\hline & & & & tef1 & $r p b 2$ & act & cal \\
\hline T. tawa & Green/harzianum & CBS 114233 = G.J.S. 97-174 & Thailand & FJ463313 & AY391956 & FJ442570 & FJ442406 \\
\hline T. pleuroti & Green/harzianum & CBS 124387 & Korea & HM142382 & HM142372 & JN133599 & JN133539 \\
\hline T. pleuroticola & Green/harzianum & CBS 124383 & Korea & HM142381 & HM142371 & JN133598 & JN133538 \\
\hline T. cinnamomeum & Green/harzianum & G.J.S. 97-237 (culture dead) & USA & AY737732 & AY391920 & FJ442582 & JN133524 \\
\hline T. stramineum & Green/harzianum & CBS 114248 = G.J.S. 02-84 & Sri Lanka & AY737746 & AY391945 & FJ442583 & FJ442386 \\
\hline T. velutinum & Green/harzianum & DAOM 230013 & Nepal & AY937415 & JN133569 & JN133614 & JN133550 \\
\hline T. tomentosum & Green/harzianum & DAOM 178713a & Canada & AY750882 & AF545557 & JN133612 & JN133548 \\
\hline T. catoptron & Green/harzianum & CBS 114232 = G.J.S. 02-76 & Sri Lanka & AY737726 & AY391900 & FJ442584 & FJ442387 \\
\hline T. afroharzianum & Green/harzianum & G.J.S. 00-24 & Mexico & AF443940 & FJ442726 & FJ442488 & AF442880 \\
\hline T. afroharzianum & Green/harzianum & G.J.S. 05-113 & Italy & FJ463378 & - & FJ442571 & FJ442371 \\
\hline T. atrobrunneum & Green/harzianum & G.J.S. 05-100 & Italy & FJ463299 & - & FJ442496 & FJ442364 \\
\hline T. atrobrunneum & Green/harzianum & G.J.S. 05-467 & UK & FJ463303 & - & FJ442501 & FJ442339 \\
\hline T. atrobrunneum & Green/harzianum & CBS 130440 = G.J.S. 04-67 & Italy & FJ463360 & FJ442724 & FJ442492 & FJ442329 \\
\hline T. atrobrunneum & Green/harzianum & CBS $548.92=$ G.J.S. $92-110$ & France & AF443942 & - & FJ442528 & AF442883 \\
\hline T. atrobrunneum & Green/harzianum & CBS $130429=$ G.J.S. 90-254 & Germany & AF443943 & FJ442735 & FJ442525 & AF442886 \\
\hline T. atrobrunneum & Green/harzianum & G.J.S. 05-101 & Italy & FJ463392 & FJ442745 & FJ442497 & FJ442331 \\
\hline T. atrobrunneum & Green/harzianum & G.J.S. 05-469 & UK & FJ463304 & - & FJ442502 & FJ442340 \\
\hline T. atrobrunneum & Green/harzianum & CBS $130495=$ G.J.S. 05-106 & Italy & FJ463297 & - & FJ442580 & FJ442332 \\
\hline T. tomentosum & Green/harzianum & DAOM 178713a & Canada & AY750882 & AF545557 & JN133612 & JN133548 \\
\hline T. inhamatum & Green/harzianum & CBS $273.78=$ IMI $287526=$ G.J.S. $95-39$ & Colombia & AF348099 & FJ442725 & FJ442561 & AF442891 \\
\hline T. inhamatum & Green/harzianum & CBS 273.78 = C.P.K. 202 ex-type & Colombia & AY605853 & - & - & FJ577683 \\
\hline$T . \mathrm{sp}$ & Green/harzianum & MMS1255 = TUCIM 5509 & France & KU758964 & MN450663 & MN450664 & MN428075 \\
\hline T. harzianum & Green/harzianum & J.B. NZ11-1 ; DAOM 233829 & New Zealand & EF191333 & - & - & FJ577764 \\
\hline T. harzianum & Green/harzianum & J.B. NZ2-4 ; DAOM 233823 & New Zealand & EF191331 & - & - & FJ577762 \\
\hline T. pollinicola & Green/harzianum & LC11682 = LF1542 & China & MF939619 & MF93964 & - & MF939586 \\
\hline T. pollinicola & Green/harzianum & LC11686 = LF2050 & China & MF939620 & MF93965 & - & MF939587 \\
\hline T. pollinicola & Green/harzianum & LC11687 =LF2051 & China & MF939621 & MF93966 & - & MF939588 \\
\hline
\end{tabular}

CBS = CBS Fungal Biodiversity Centre culture collection, the Netherlands

DAOM. = Agriculture and Agri-Food Canada National Mycological Culture Collection

IMI. = CABI culture collection, UK

ATCC. = American Type Culture Collection, Manassas, Virginia, USA

BPI = US National Fungus Collection

G.J.S. = G. J. Samuels

Hypo, C.P.K. and $S=$ W. Jaklitsch collection numbers

Dis $=$ H.C. Evans endophyte cultures

LA and $T=P$. Chaverri endophyte cultures

MMS = marine fungal collection, University of Nantes

TUCIM = TU Wien Collection of Industrially Important Microorganisms

LC: working collection of Lei Cai, housed at the Institute of Microbiology, Chinese Academy of Sciences, Beijing, China 
Fig. S2. Phylogram of the best maximum likelihood tree (log likelihood: -27559.25) of tefl and $r p b 2$, which includes species from the different clade described by Jaklitsch and Voglmayr (2015). Values at nodes represent ML bootstrap (MLBP)/MP bootstrap (MPBP). The tree is drawn to scale, with branch lengths measured in the number of substitutions per site. The analysis involved 112 nucleotide sequences. There were a total of 2366 positions in the final dataset. Evolutionary analyses were conducted in MEGA7 (Kumar et al., 2016).

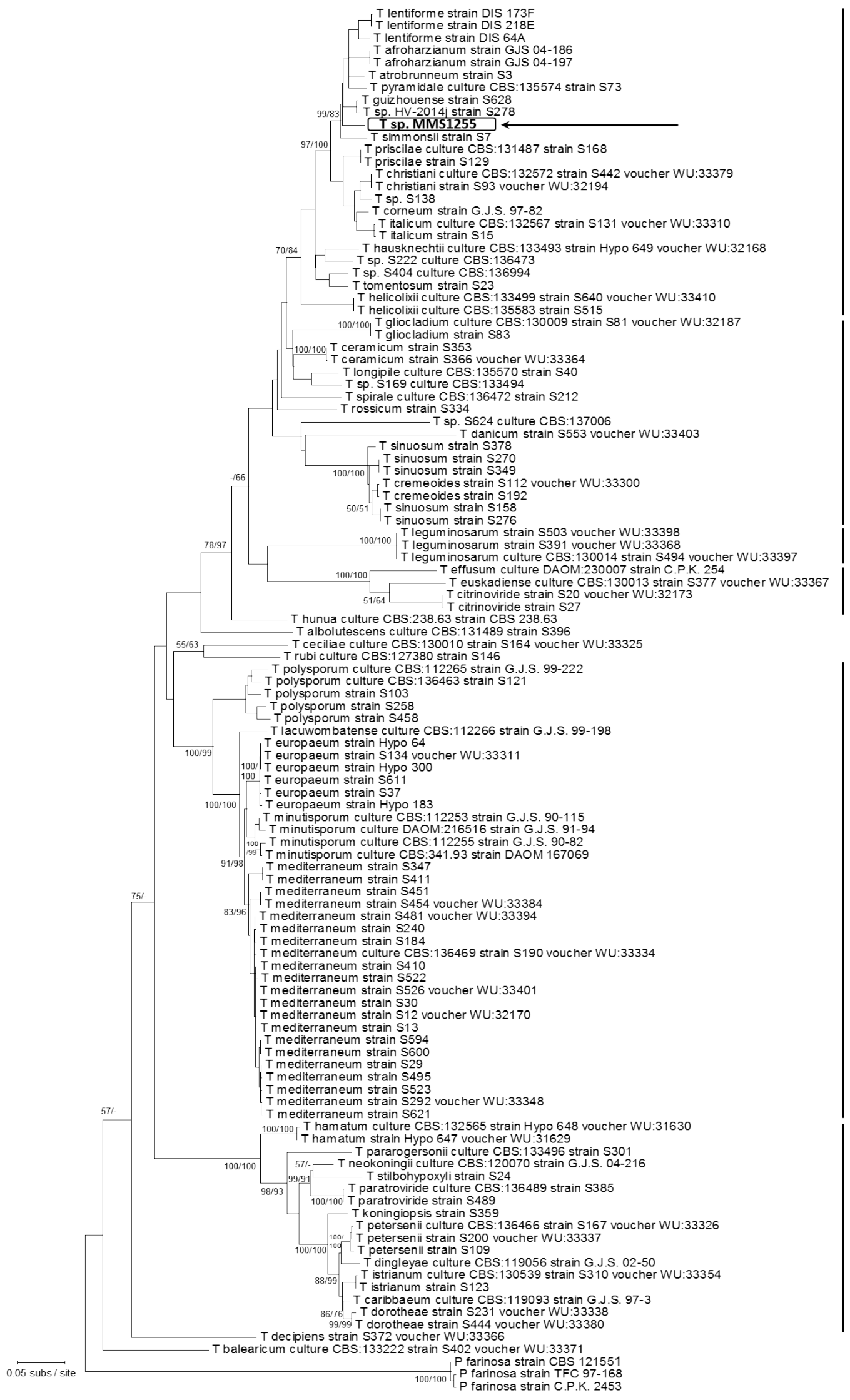

Green/harzianum clade

aff. Longibrachiatum clade

Longibrachiatum clade

Polysporum clade 
3/ ESI-MS profiling of peptaibol-enriched fraction of Trichoderma sp. MMS1255

Fig. S3. Superposition of positive ESI-MS spectra (zoomscan mode) of known 14-res peptaibols (orange) and peptaibol-enriched fraction from Trichderma sp. MMS1255 (black).

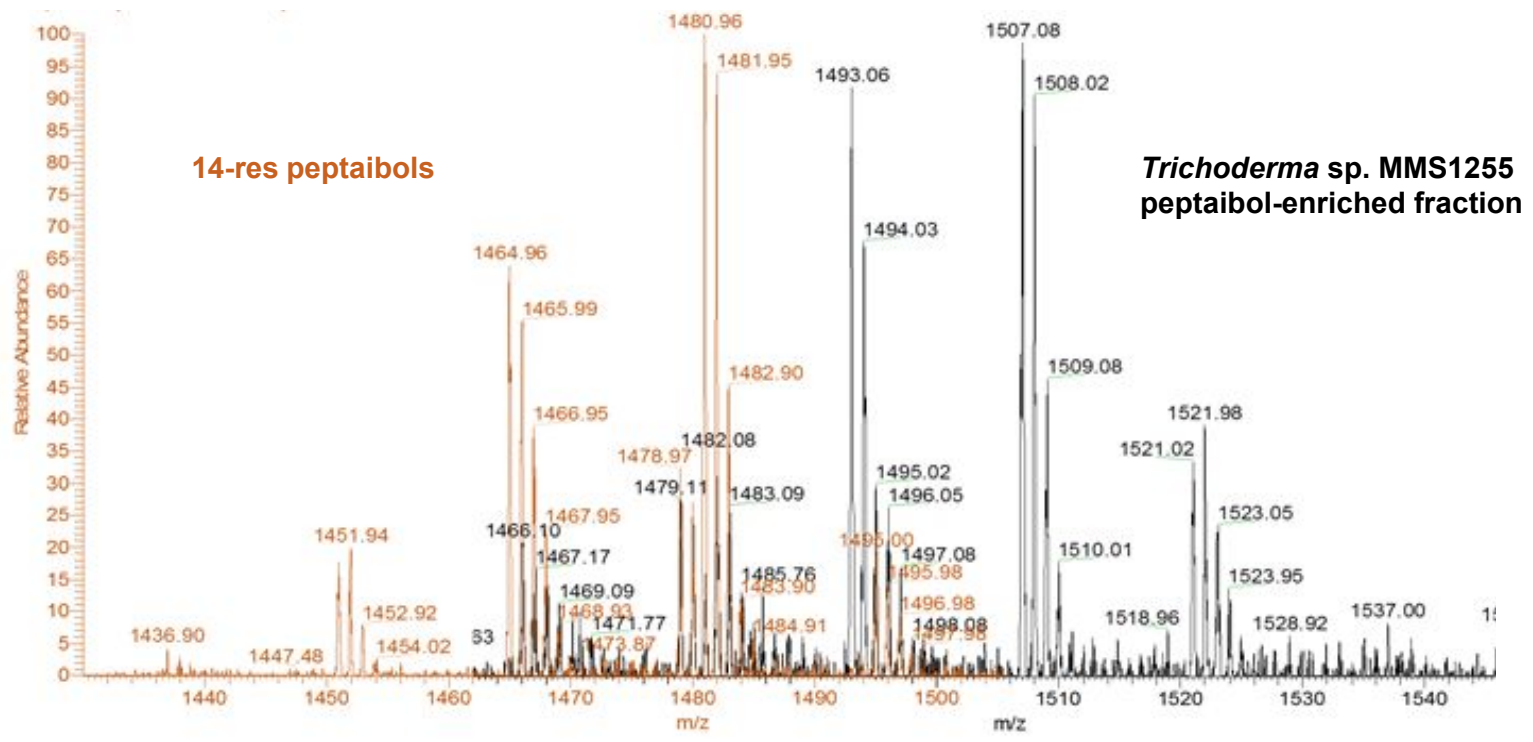


2/ ESI-MS ${ }^{2}$ fragmentation of pentadecaibins I-V (1-5)

Table S2. Diagnostic fragment ions of pentacaibins I-V (1-5) from the strain Trichoderma sp. MMS 1255

\begin{tabular}{|c|c|c|c|c|c|}
\hline Pentadecaibin & -1 & - -II & - III & -IV & $-\mathbf{V}$ \\
\hline HPLC fractions 2-10- & 4 & 5 & 6 & 7 & 8 \\
\hline$t_{\mathrm{R}}[\mathrm{min}]$ & $27.0-30.0$ & $30.0-32.0$ & $32.0-35.5$ & $35.5-39.0$ & $39.0-41.0$ \\
\hline Diagnostic fragment ion & Peak $[\mathrm{m} / \mathrm{z}]$ & & & & \\
\hline$[M+\mathrm{Na}]^{+}$ & 1478.8 & 1492.8 & 1492.8 & 1506.8 & 1506.8 \\
\hline$[\mathrm{M}+2 \mathrm{Na}]^{2+}$ & 750.9 & 757.9 & 757.9 & 764.9 & 764.9 \\
\hline$\left[\mathrm{M}+\mathrm{Na}-\mathrm{H}_{2} \mathrm{O}\right]^{+}$ & 1460.8 & 1474.8 & 1474.8 & 1488.8 & 1488.8 \\
\hline$[M+\mathrm{H}]^{+}$ & 1456.8 & 1470.8 & 1470.8 & 1484.8 & 1484.8 \\
\hline$b_{5}$ & 476.2 & 476.2 & n.d. & 476.2 & n.d. \\
\hline$b_{6}$ & 604.3 & 604.3 & 604.3 & 604.3 & 618.3 \\
\hline$b_{7}$ & 689.3 & 689.3 & 703.3 & 703.3 & 717.3 \\
\hline$b_{8}$ & 788.4 & 802.4 & 802.4 & 816.4 & 816.4 \\
\hline$b_{9}$ & 873.5 & 887.5 & 887.5 & 901.5 & 901.5 \\
\hline$b_{10}$ & 944.5 & 958.5 & 958.5 & 972.5 & 972.5 \\
\hline$b_{11}$ & 1029.5 & 1043.5 & 1043.5 & 1057.5 & 1057.5 \\
\hline$b_{12}$ & 1114.6 & 1128.6 & 1128.6 & 1142.6 & 1142.6 \\
\hline$b_{13}$ & 1199.6 & 1213.6 & 1213.6 & 1227.6 & 1227.6 \\
\hline$b_{14}$ & 1327.7 & 1341.7 & 1341.7 & 1355.7 & 1355.7 \\
\hline$a_{5}$ & 448.2 & 448.2 & 448.2 & 448.2 & 462.2 \\
\hline$a_{6}$ & 576.3 & 576.3 & 576.3 & 576.3 & 590.3 \\
\hline$a_{7}$ & 661.3 & 661.3 & 675.3 & 675.3 & 689.3 \\
\hline$a_{8}$ & 760.4 & 774.4 & 774.4 & 788.4 & 788.4 \\
\hline$a_{9}$ & 845.5 & 859.5 & 859.5 & 873.5 & 873.5 \\
\hline$a_{10}$ & 916.5 & 930.5 & 930.5 & 944.5 & 944.5 \\
\hline$a_{11}$ & 1001.5 & 1015.5 & 1015.5 & 1029.5 & 1029.5 \\
\hline$a_{12}$ & 1086.6 & 1100.6 & 1100.6 & 1114.6 & 1114.6 \\
\hline$a_{13}$ & 1171.6 & 1185.6 & 1185.6 & 1199.6 & 1199.6 \\
\hline$a_{14}$ & 1299.7 & 1313.7 & 1313.7 & 1327.7 & 1327.7 \\
\hline$y_{7 P}$ & 713.4 & 713.4 & 713.4 & 713.4 & 713.4 \\
\hline$y_{8 P}$ & 812.5 & 826.5 & 812.5 & 826.5 & 812.5 \\
\hline$y_{9 P}$ & 897.5 & 911.5 & 911.5 & 925.5 & 911.5 \\
\hline$y_{10 P}$ & 1025.6 & 1039.6 & 1039.6 & 1053.6 & 1039.6 \\
\hline$y_{11 P}$ & 1110.7 & 1124.7 & 1124.7 & 1138.7 & 1138.7 \\
\hline$y_{12 P}$ & 1223.7 & 1237.7 & 1237.7 & 1251.7 & 1251.7 \\
\hline$y_{13 P}$ & 1294.8 & 1308.8 & 1308.8 & 1322.8 & 1322.8 \\
\hline$y_{14 P}$ & 1351.8 & 1365.8 & 1365.8 & 1379.8 & 1379.8 \\
\hline$[M-\mathrm{H}]^{-}$ & 1454.8 & 1468.8 & 1468.8 & 1482.8 & 1482.8 \\
\hline$\left[M-\mathrm{H}^{-} \mathrm{H}_{2} \mathrm{O}\right]^{-}$ & 1436.8 & 1450.8 & 1450.8 & 1464.8 & 1464.8 \\
\hline$[M-\mathrm{H}-\mathrm{Ac}]^{-}$ & 1412.8 & 1426.8 & 1426.8 & 1440.8 & 1440.8 \\
\hline$y_{4 N}$ & 448.3 & 448.3 & 448.3 & 448.3 & 448.3 \\
\hline$y_{5 \mathrm{~N}}$ & 533.3 & 533.3 & 533.3 & 533.3 & 533.3 \\
\hline$y_{6 \mathrm{~N}}$ & 604.4 & 604.4 & 604.4 & 604.4 & 604.4 \\
\hline$y_{7 \mathrm{~N}}$ & 689.4 & 689.4 & 689.4 & 689.4 & 689.4 \\
\hline$y_{8 \mathrm{~N}}$ & 788.5 & 802.5 & 788.5 & 802.5 & 788.5 \\
\hline$y_{9 \mathrm{~N}}$ & 873.5 & 887.5 & 887.5 & 901.5 & 887.5 \\
\hline$y_{10 \mathrm{~N}}$ & 1001.6 & 1015.6 & 1015.6 & 1029.6 & 1015.6 \\
\hline$y_{11 \mathrm{~N}}$ & 1086.7 & 1100.7 & 1100.7 & 1114.7 & 1114.7 \\
\hline$y_{12 \mathrm{~N}}$ & 1199.7 & 1213.7 & 1213.7 & 1227.7 & 1227.7 \\
\hline$y_{13 \mathrm{~N}}$ & 1270.8 & 1284.8 & 1284.8 & 1298.8 & 1298.8 \\
\hline$y_{14 \mathrm{~N}}$ & 1327.8 & 1341.8 & 1341.8 & 1355.8 & 1355.8 \\
\hline
\end{tabular}


Fig. S4. Pentadecaibin II (2) ESI-MS ${ }^{2}$ spectrum at $m / z 1492.8[\mathrm{M}+\mathrm{Na}]^{+}$(positive mode)

(+) $M S^{2}[1492.8]$

$\left[\mathrm{M}+\mathrm{Na}-\mathrm{H}_{2} \mathrm{O}\right]^{+}$

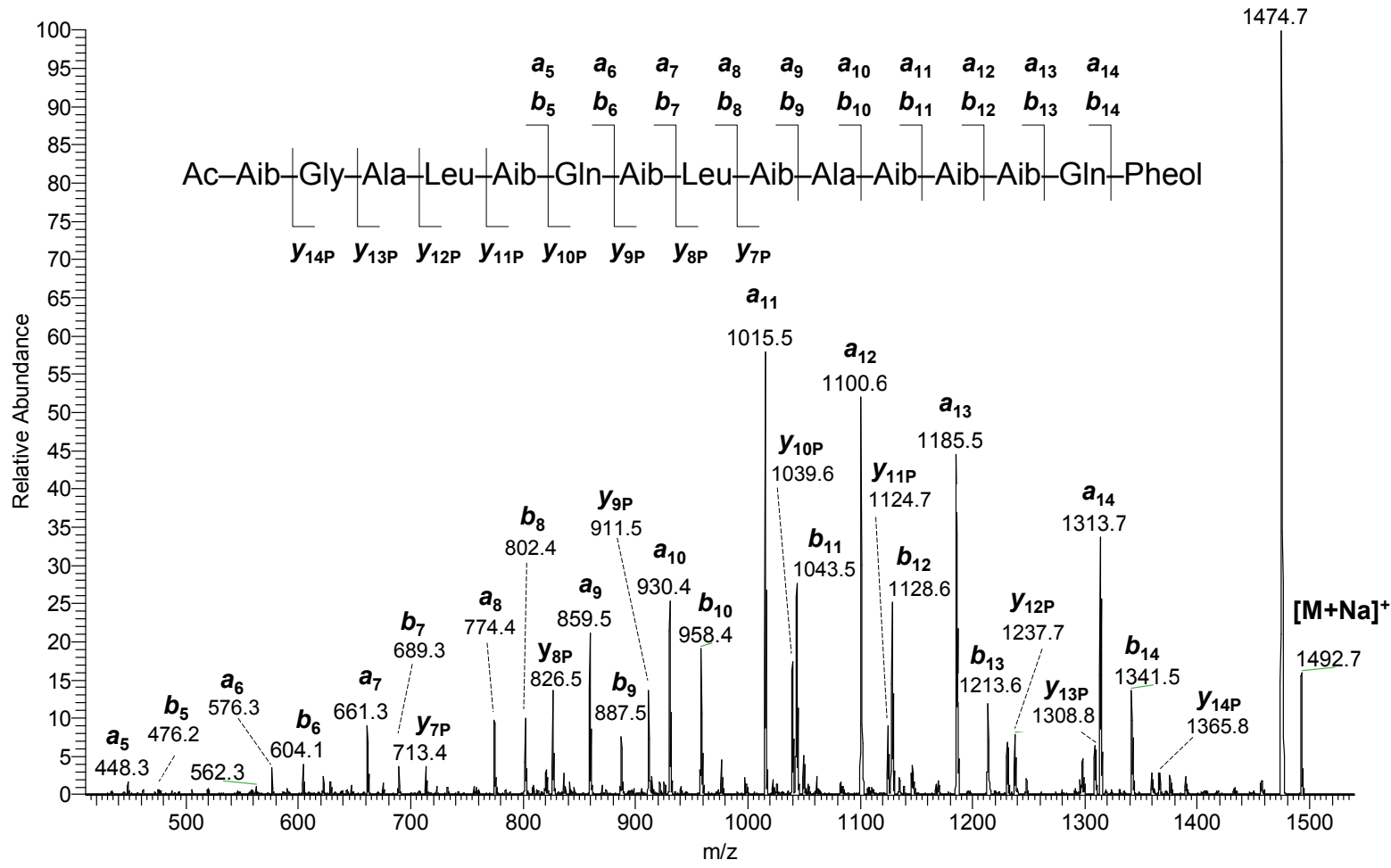

Fig. S5. Pentadecaibin II (2) ESI-MS ${ }^{2}$ spectrum at $m / z 1468.8[\mathrm{M}-\mathrm{H}]^{-}$(negative mode)

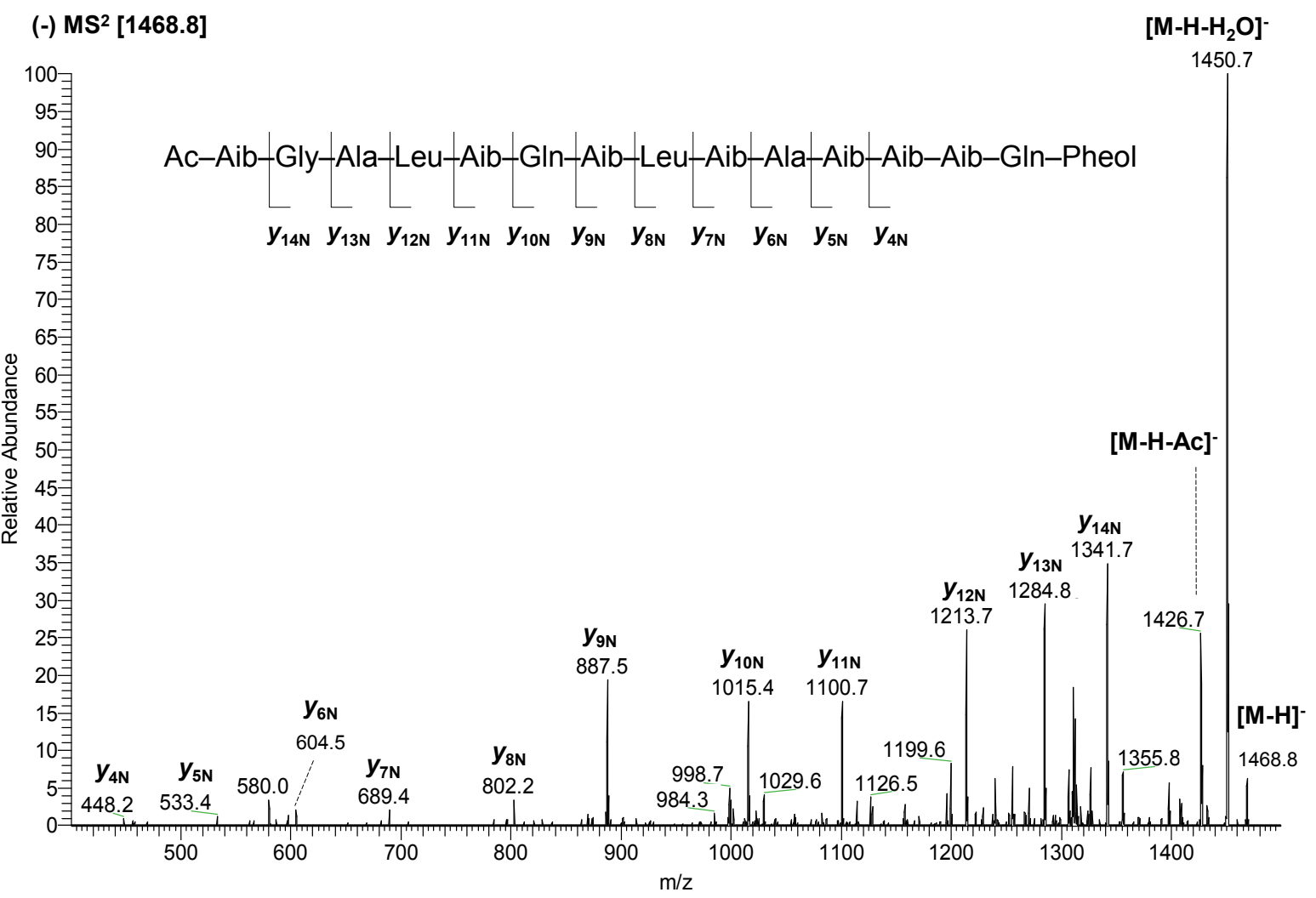


Fig. S6. Pentadecaibin III (3) ESI-MS ${ }^{2}$ spectrum at $\mathrm{m} / z 1492.8[\mathrm{M}+\mathrm{Na}]^{+}$(positive mode)

(+) $\mathrm{MS}^{2}[1492.8]$

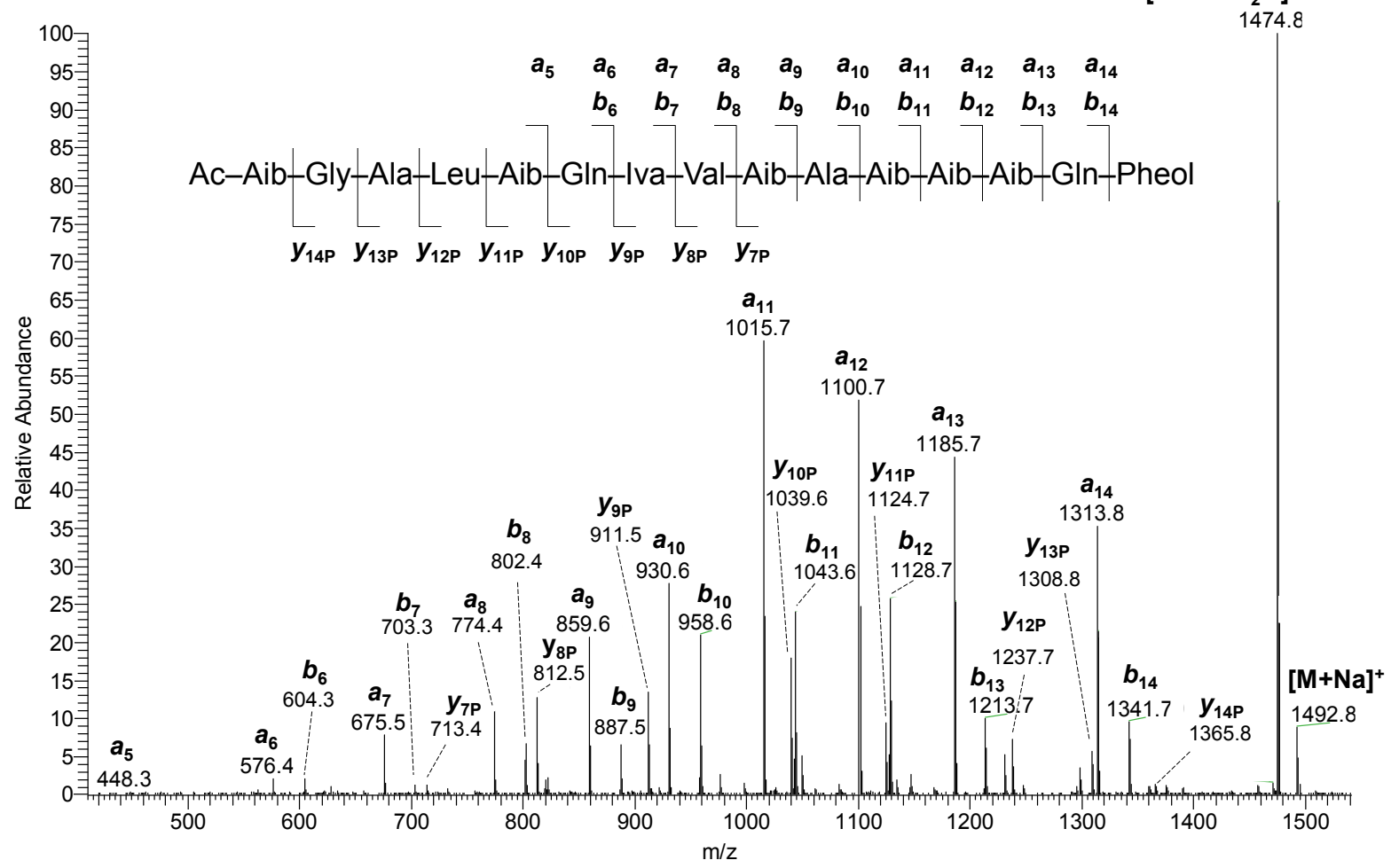

Fig. S7. Pentadecaibin III (3) ESI-MS ${ }^{2}$ spectrum at $m / z$ 1468.8 [M-H]- (negative mode)

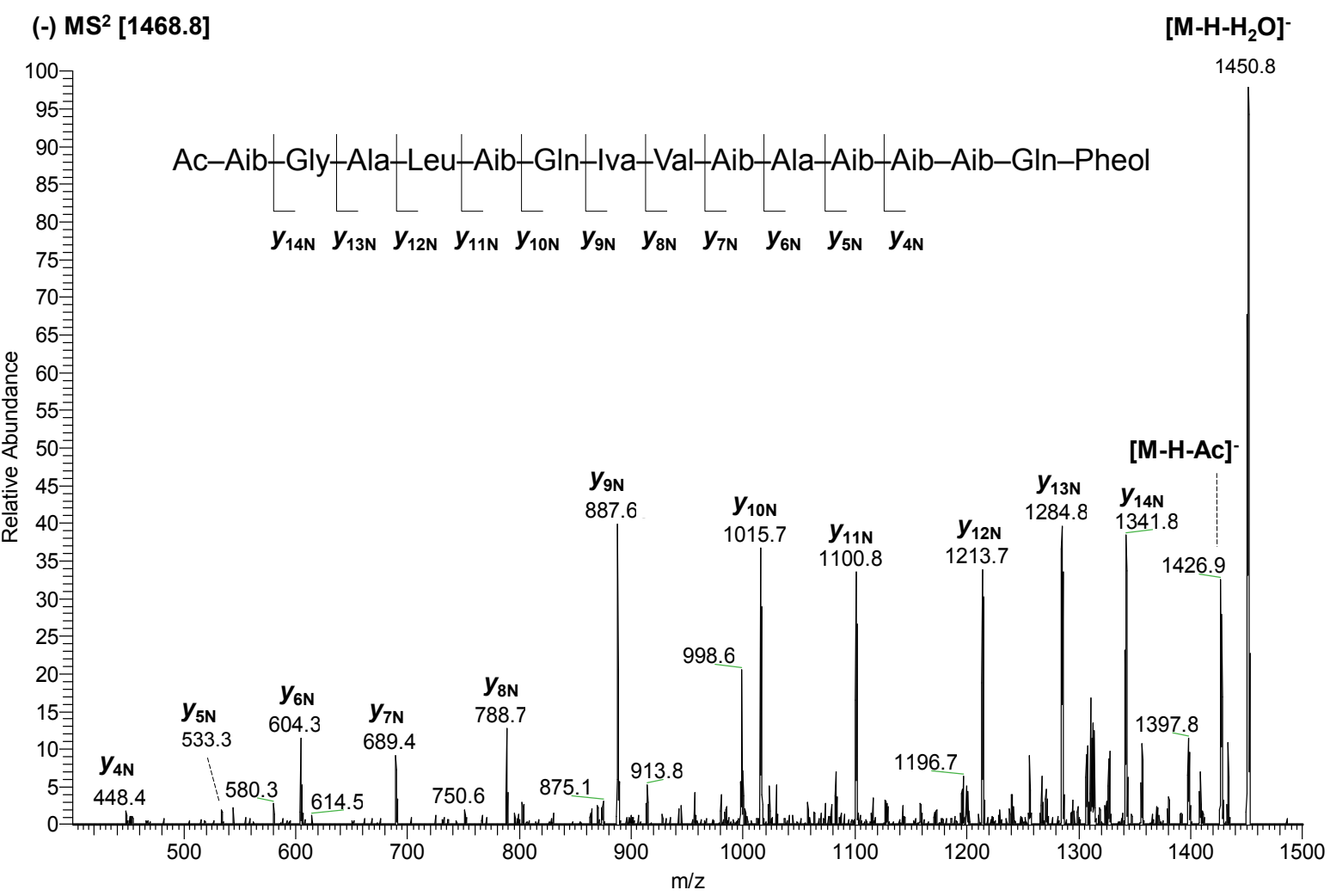


Fig. S8. Pentadecaibin IV (4) ESI-MS ${ }^{2}$ spectrum at $m / z 1506.8[\mathrm{M}+\mathrm{Na}]^{+}$(positive mode)

(+) $\mathrm{MS}^{2}[1506.8]$

$\left[\mathrm{M}+\mathrm{Na}-\mathrm{H}_{2} \mathrm{O}\right]^{+}$

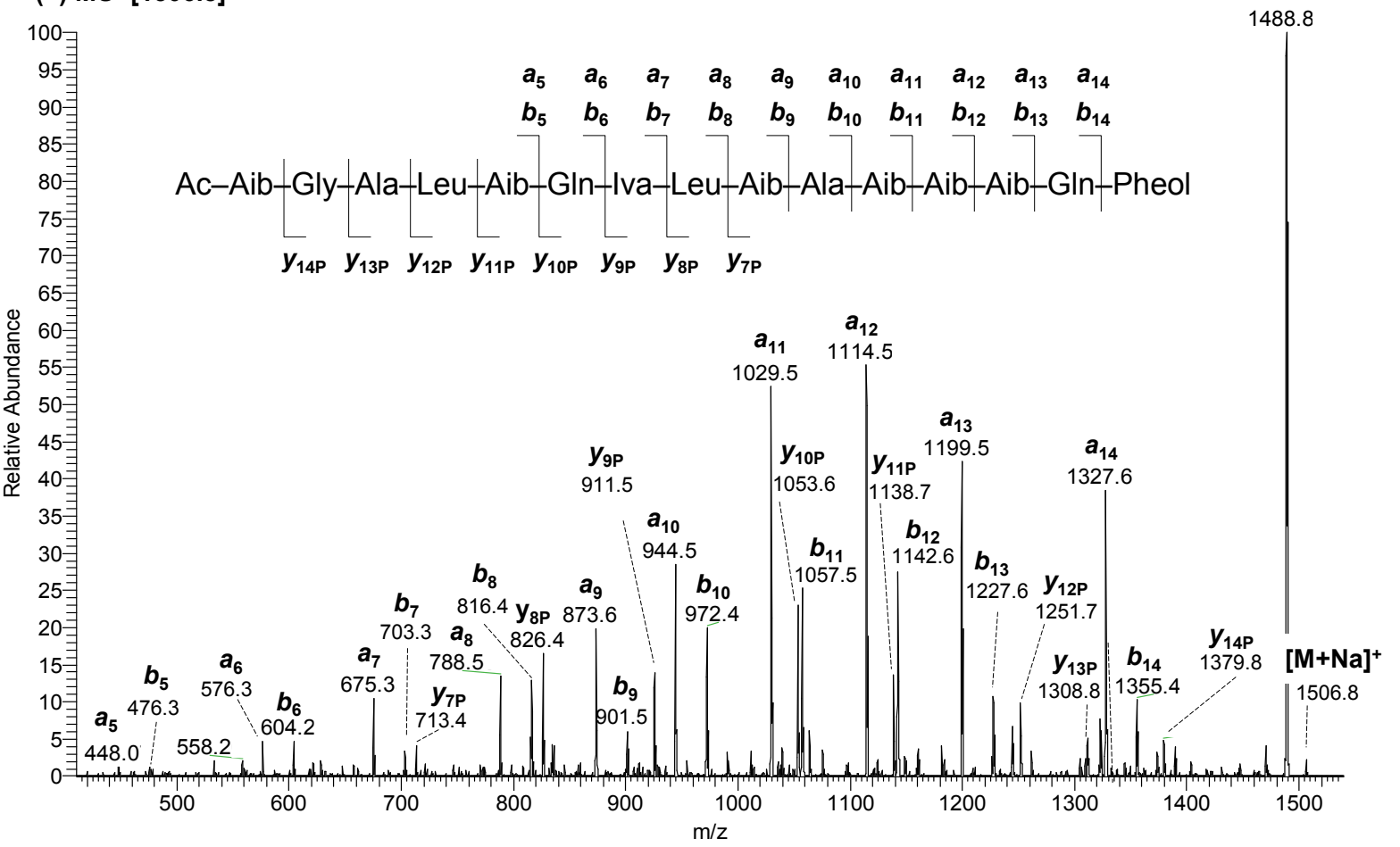

Fig. S9. Pentadecaibin IV (4) ESI-MS ${ }^{2}$ spectrum at $m / z 1482.8[\mathrm{M}-\mathrm{H}]^{-}$(negative mode)

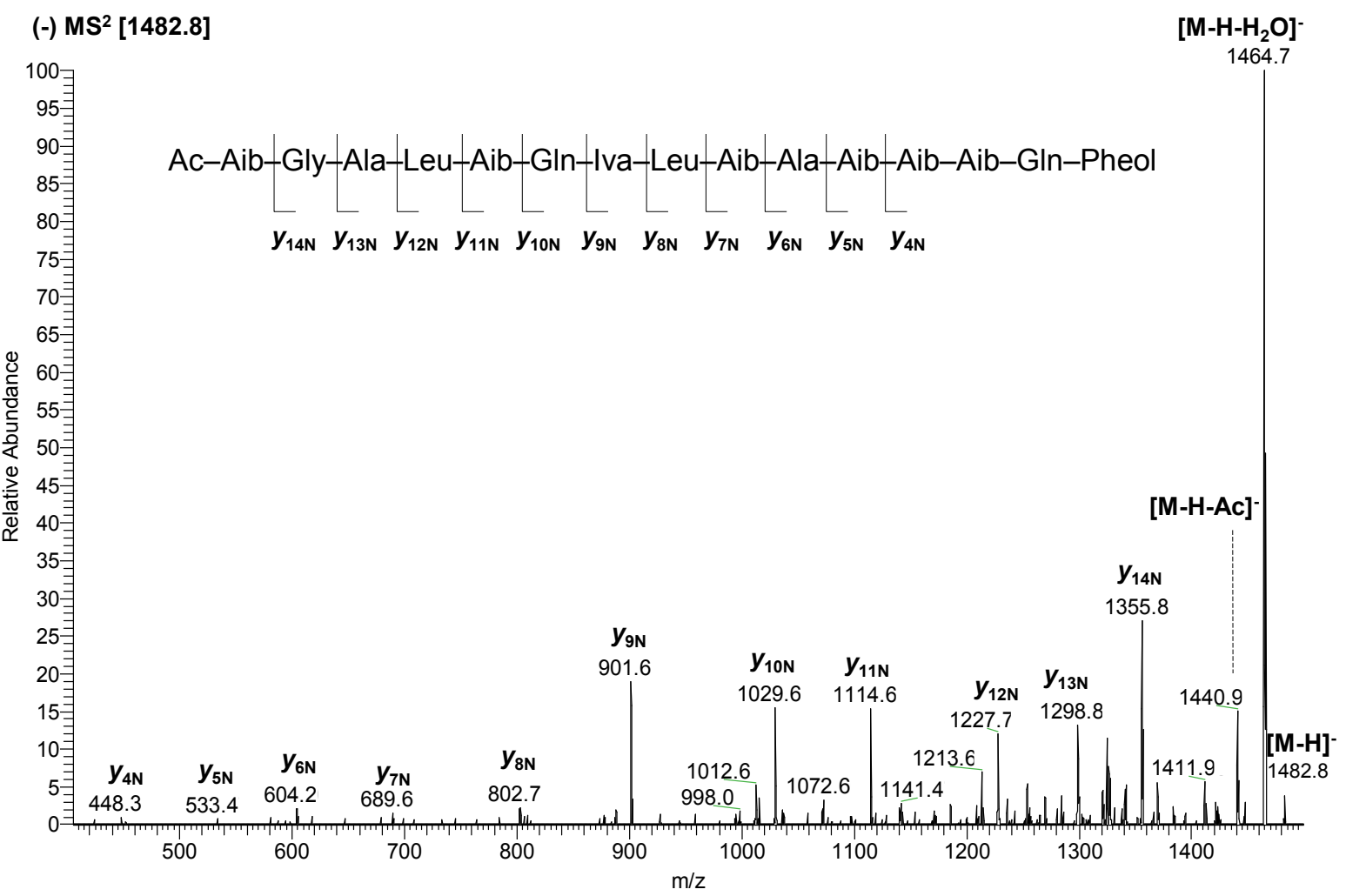


Fig. S10. Pentadecaibin V (5) ESI-MS ${ }^{2}$ spectrum at $m / z 1506.8[\mathrm{M}+\mathrm{Na}]^{+}$(positive mode)

(+) $M^{2}[1506.8]$

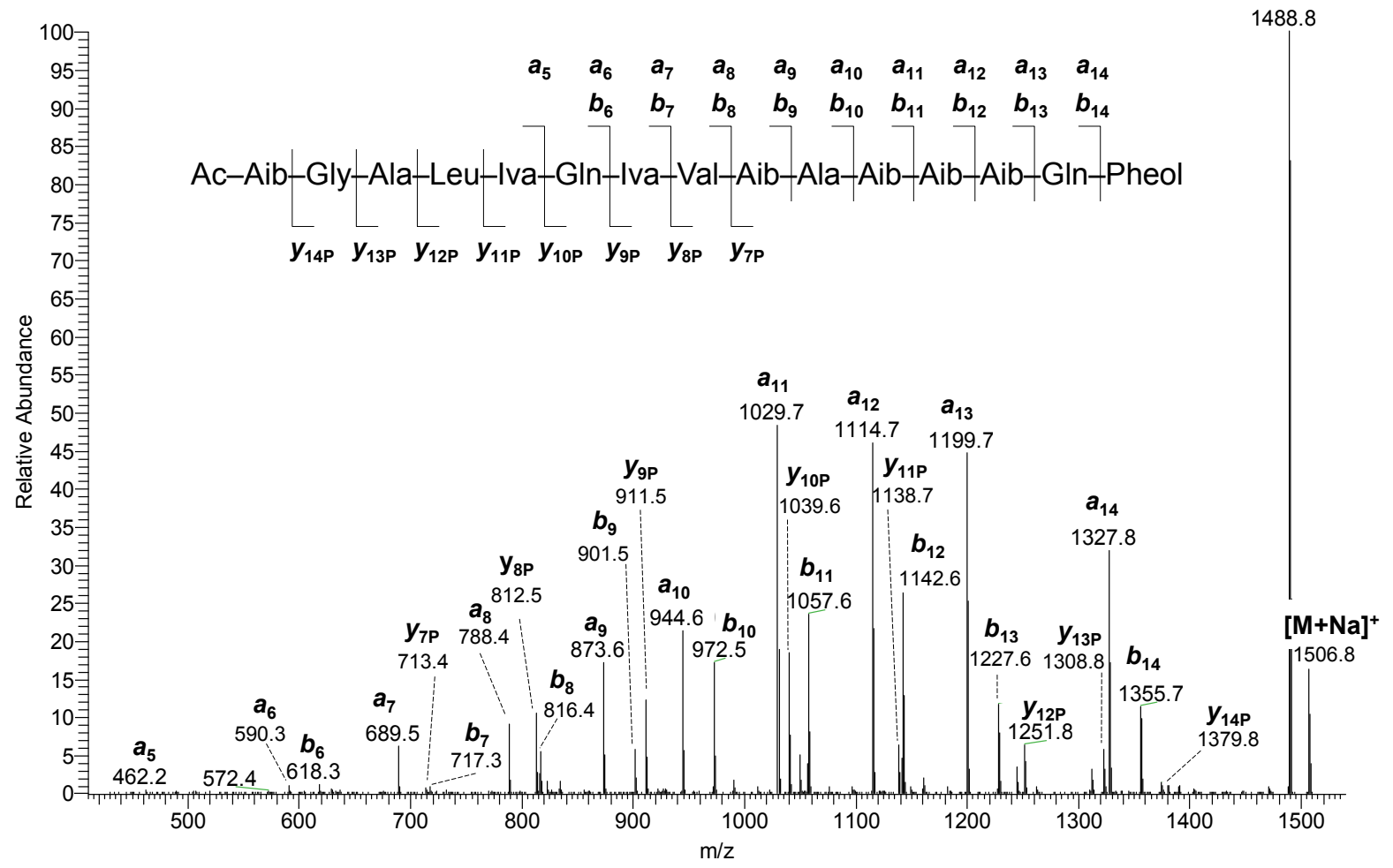

Fig S11. Pentadecaibin V (5) ESI-MS ${ }^{2}$ spectrum at m/z 1482.8 [M-H] ' (negative mode)

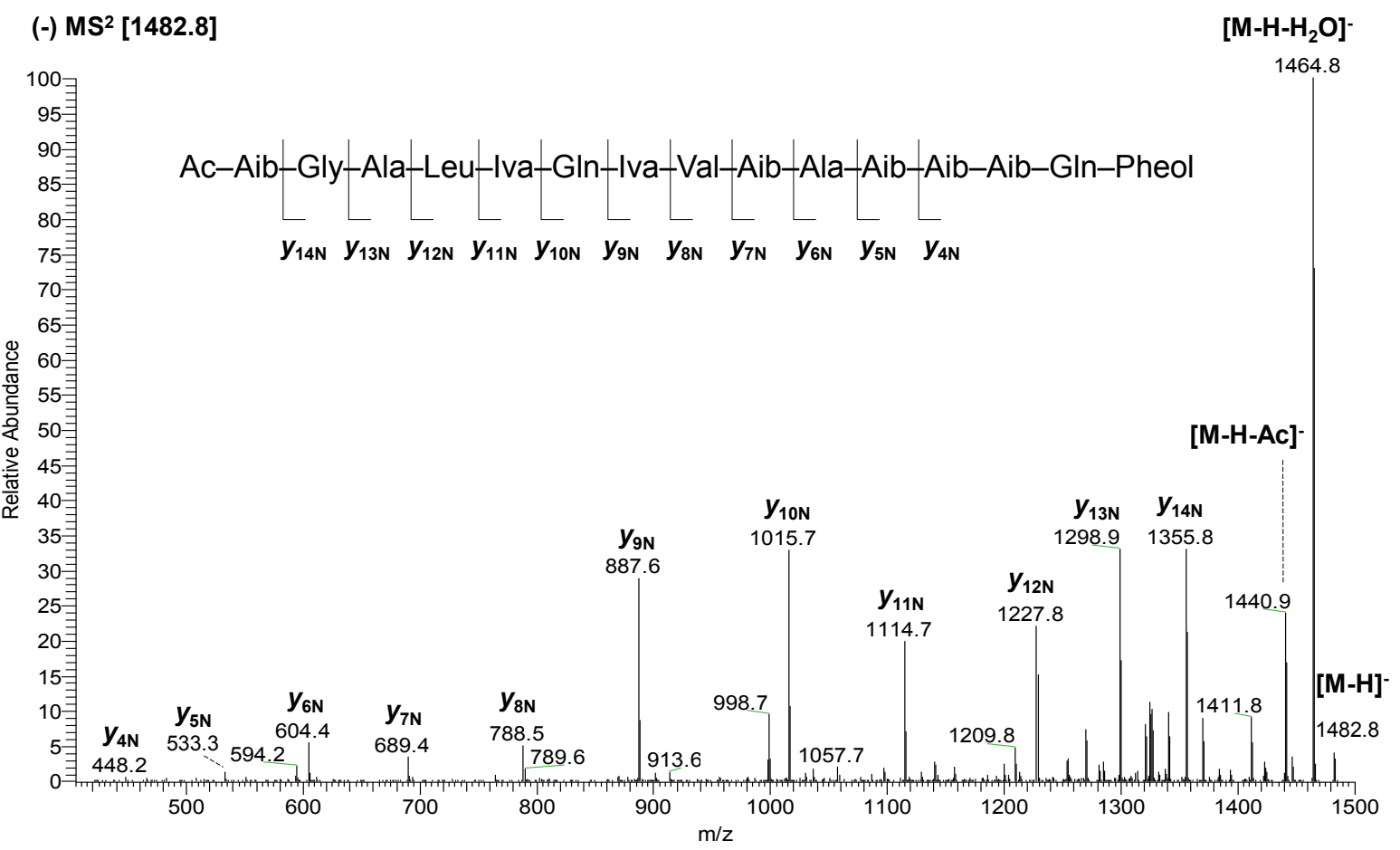


3/ Spectral data of pentadecaibins I-V (1-5)

Fig. S12. ${ }^{1} \mathrm{H}$ NMR spectrum of pentadecaibin I (1) (600 MHz, DMSO-d6, $\left.298^{\circ} \mathrm{K}\right)$

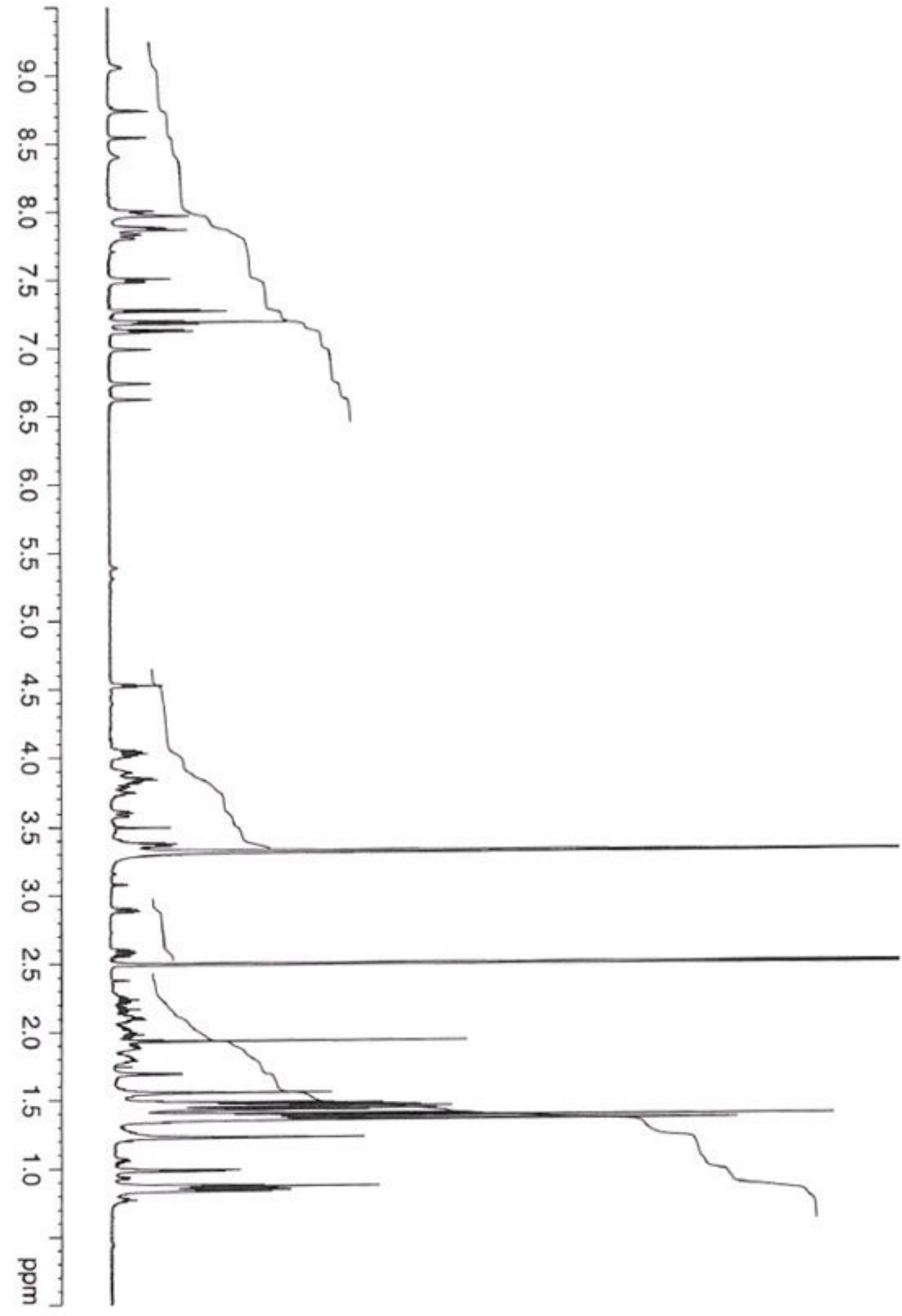


Fig. S13. ${ }^{1} \mathrm{H},{ }^{1} \mathrm{H}$ COSY spectrum of pentadecaibin I (1) $\left(600 \mathrm{MHz}, \mathrm{DMSO}-d 6,298{ }^{\circ} \mathrm{K}\right)$

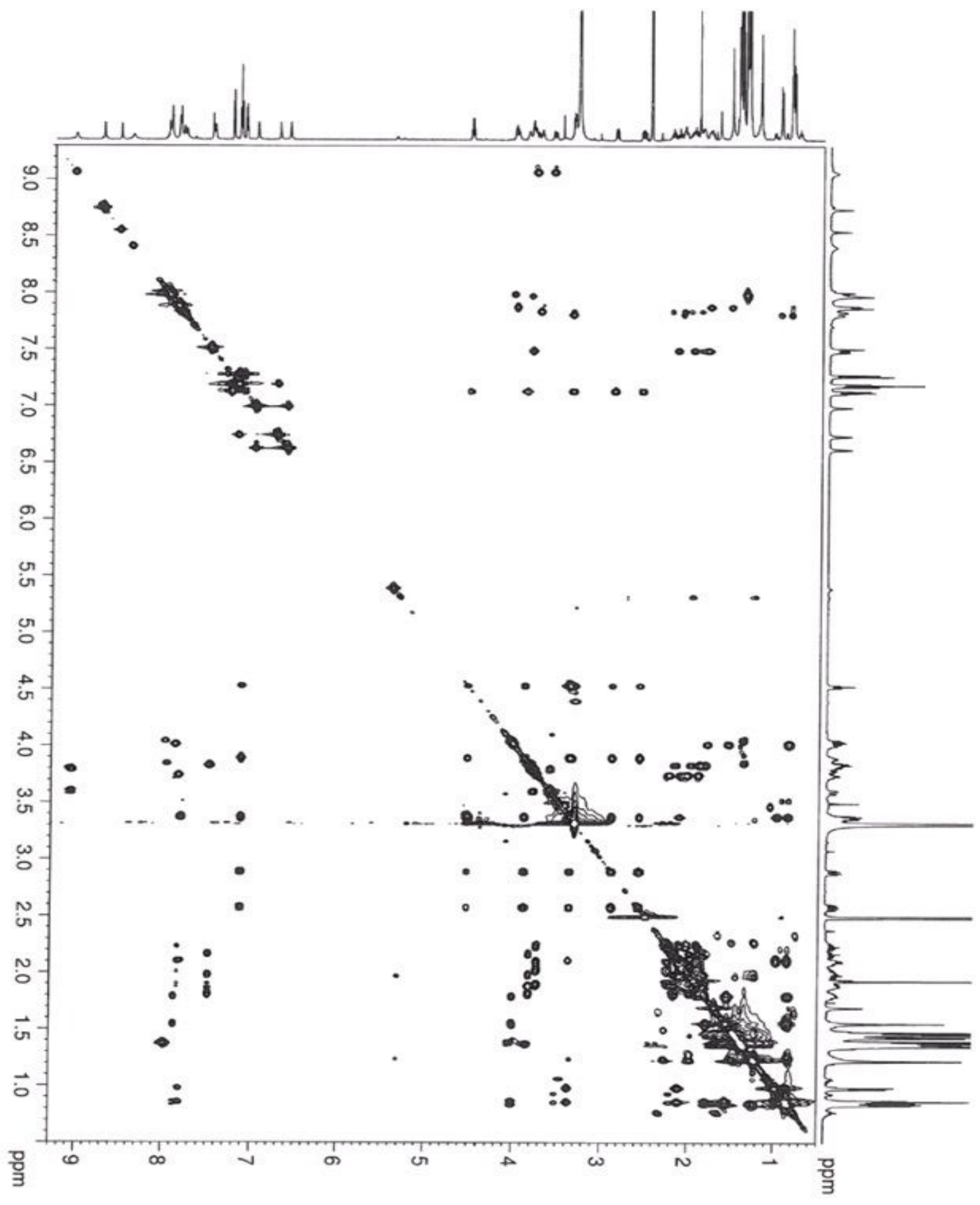


Fig. S14. ${ }^{1} \mathrm{H},{ }^{1} \mathrm{H}$ TOCSY spectrum of pentadecaibin I (1) $\left(600 \mathrm{MHz}, \mathrm{DMSO}-d 6,298{ }^{\circ} \mathrm{K}\right)$

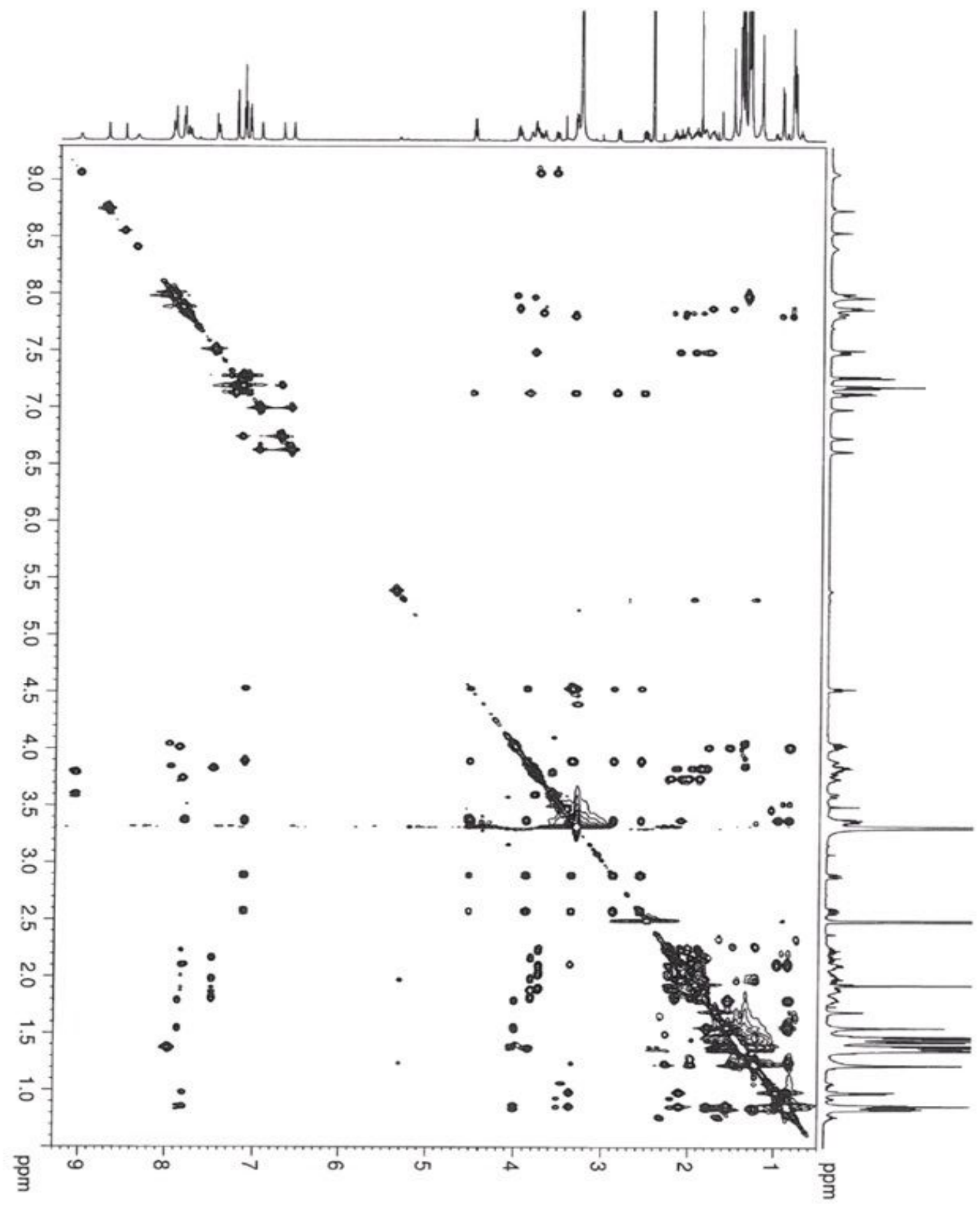


Fig. S15. ${ }^{1} \mathrm{H},{ }^{1} \mathrm{H}$ NOESY spectrum of pentadecaibin I (1) (600 MHz, DMSO-d6, $\left.298{ }^{\circ} \mathrm{K}\right)$

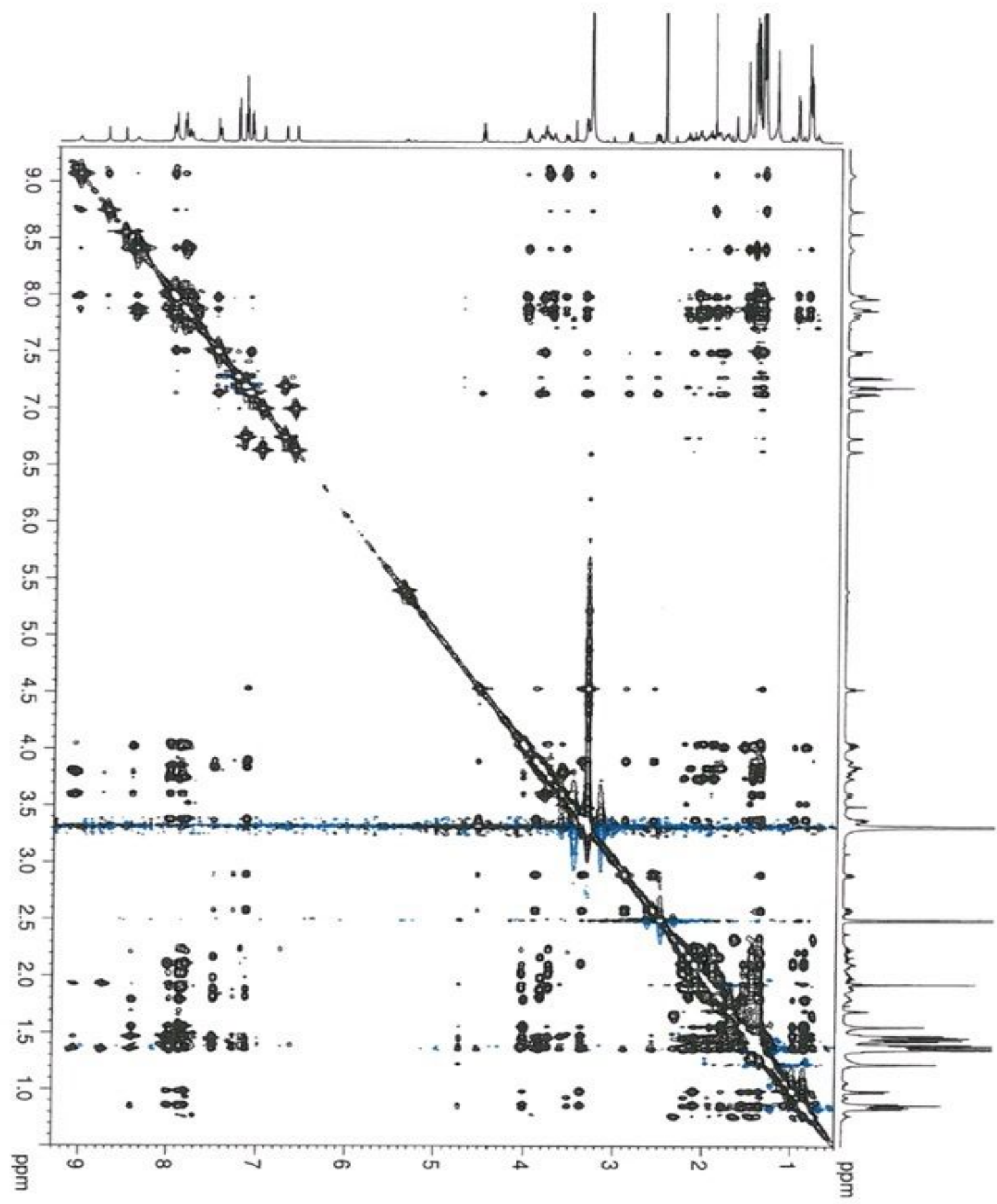


Fig. S16. DEPT-135 spectrum of pentadecaibin I (1) (150 MHz, DMSO- $\left.d 6,298^{\circ} \mathrm{K}\right)$

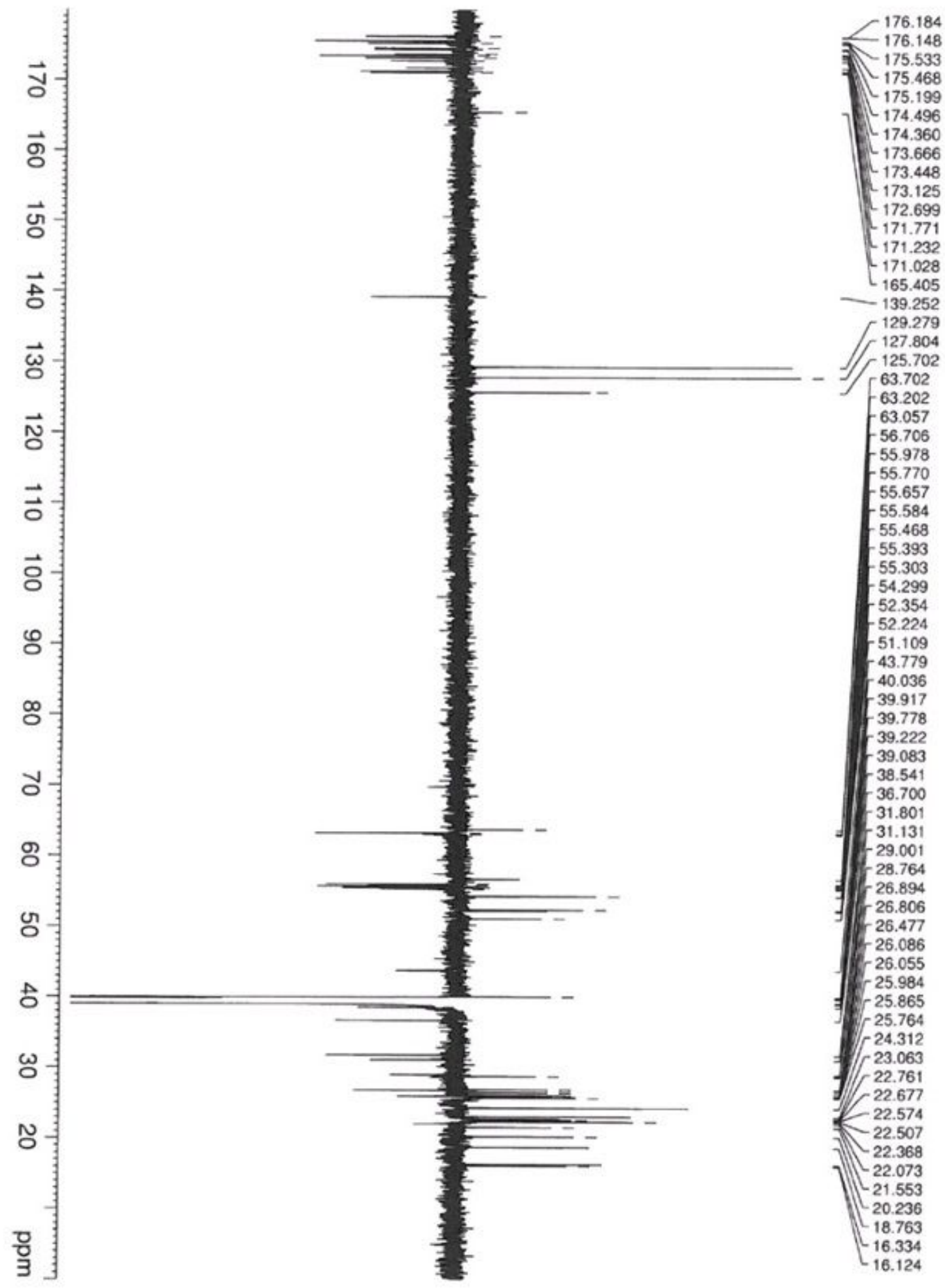


Fig. S17. ${ }^{1} \mathrm{H},{ }^{13} \mathrm{C}$ HSQC spectrum of pentadecaibin I (1) $\left(600 \mathrm{MHz}, \mathrm{DMSO}-d 6,298{ }^{\circ} \mathrm{K}\right)$

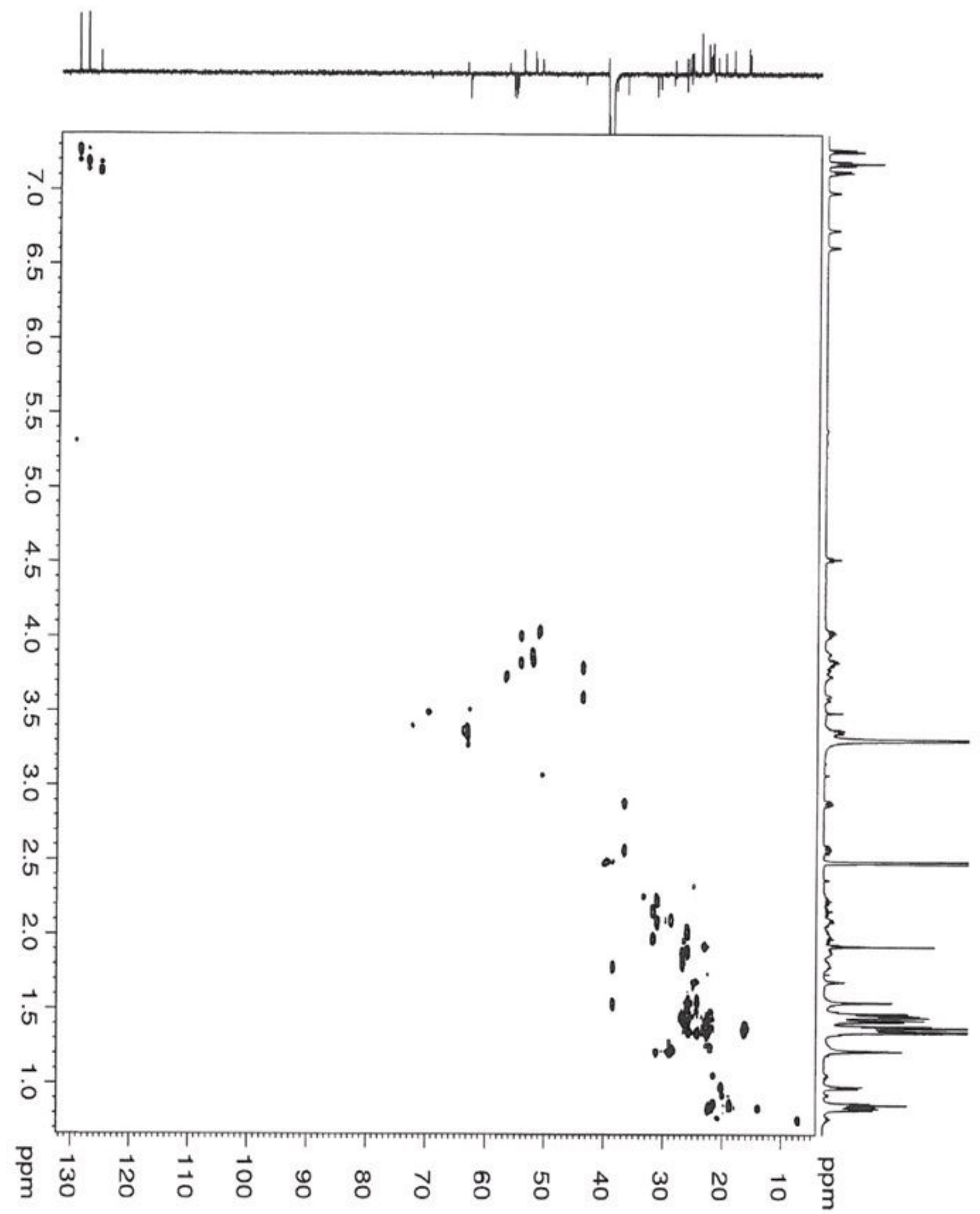


Fig. S18. ${ }^{1} \mathrm{H},{ }^{13} \mathrm{C}$ HMBC spectrum of pentadecaibin I (1) $\left(600 \mathrm{MHz}, \mathrm{DMSO}-d 6,298{ }^{\circ} \mathrm{K}\right)$




Fig. S19. CD spectrum of pentadecaibin I (1) in $\mathrm{MeOH}$

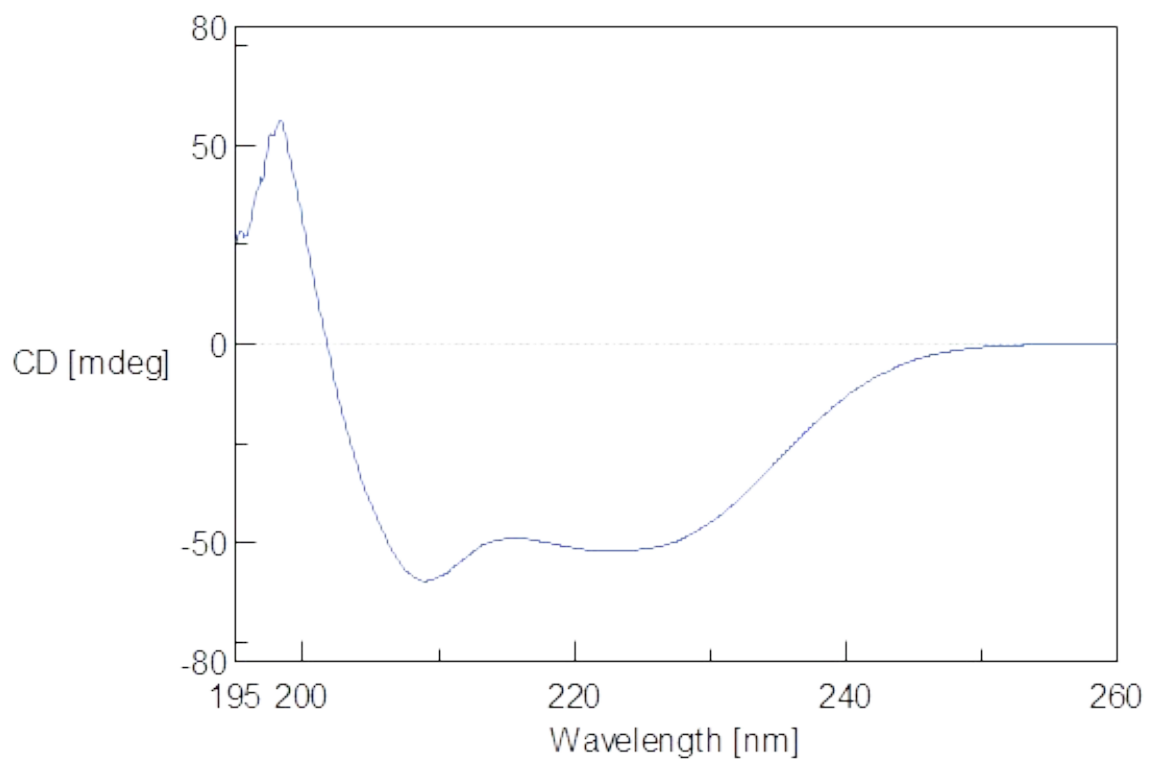


Fig. S20. ${ }^{1} \mathrm{H}$ NMR spectrum of pentadecaibin II (2) (600 MHz, DMSO-d6, $\left.298^{\circ} \mathrm{K}\right)$

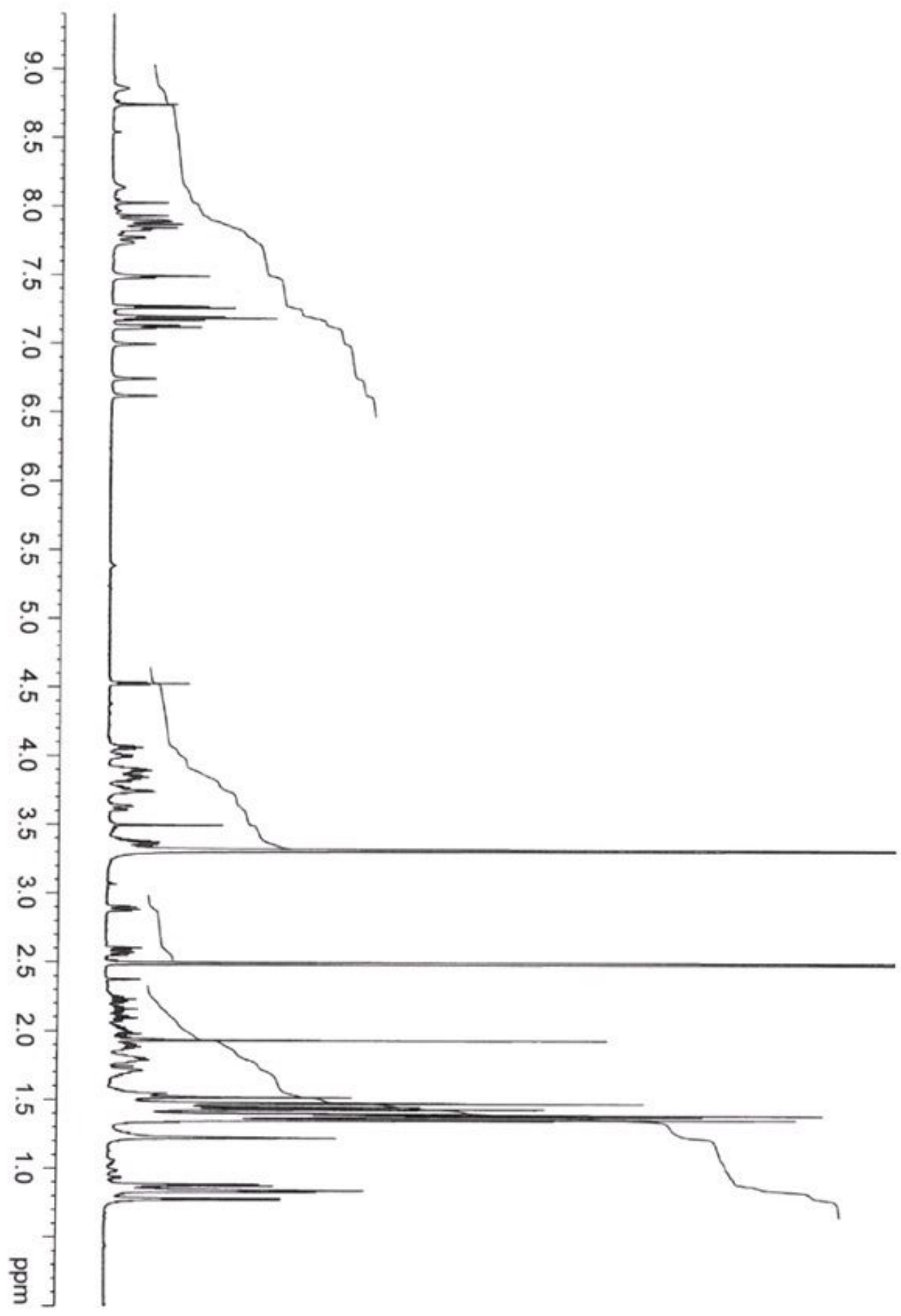


Fig. S21. ${ }^{1} \mathrm{H},{ }^{1} \mathrm{H}$ COSY spectrum of pentadecaibin II (2) (600 MHz, DMSO- $\left.d 6,298{ }^{\circ} \mathrm{K}\right)$

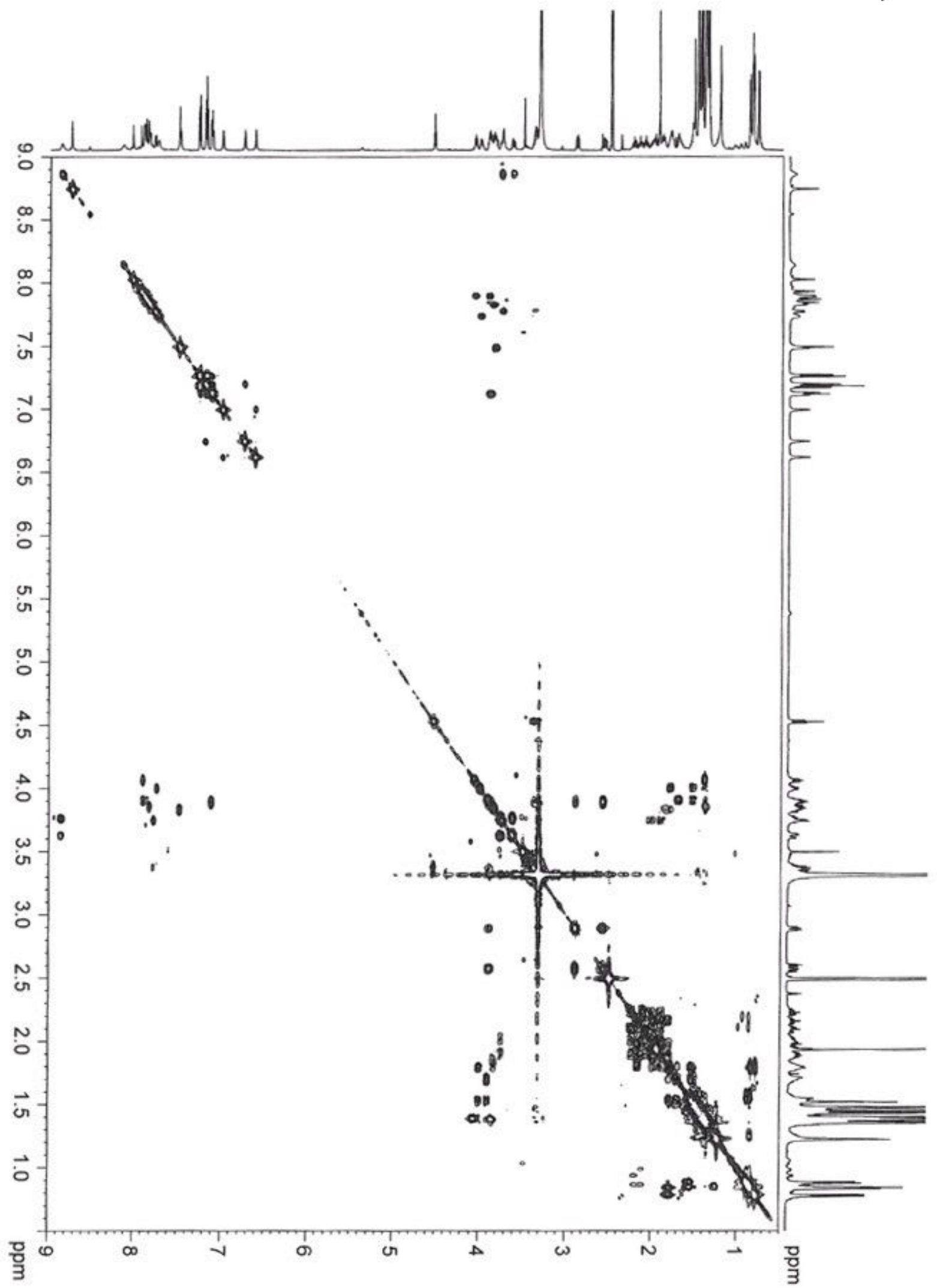


Fig. S22. ${ }^{1} \mathrm{H},{ }^{1} \mathrm{H}$ TOCSY spectrum of pentadecaibin II (2) $\left(600 \mathrm{MHz}, \mathrm{DMSO}-d 6,298{ }^{\circ} \mathrm{K}\right)$

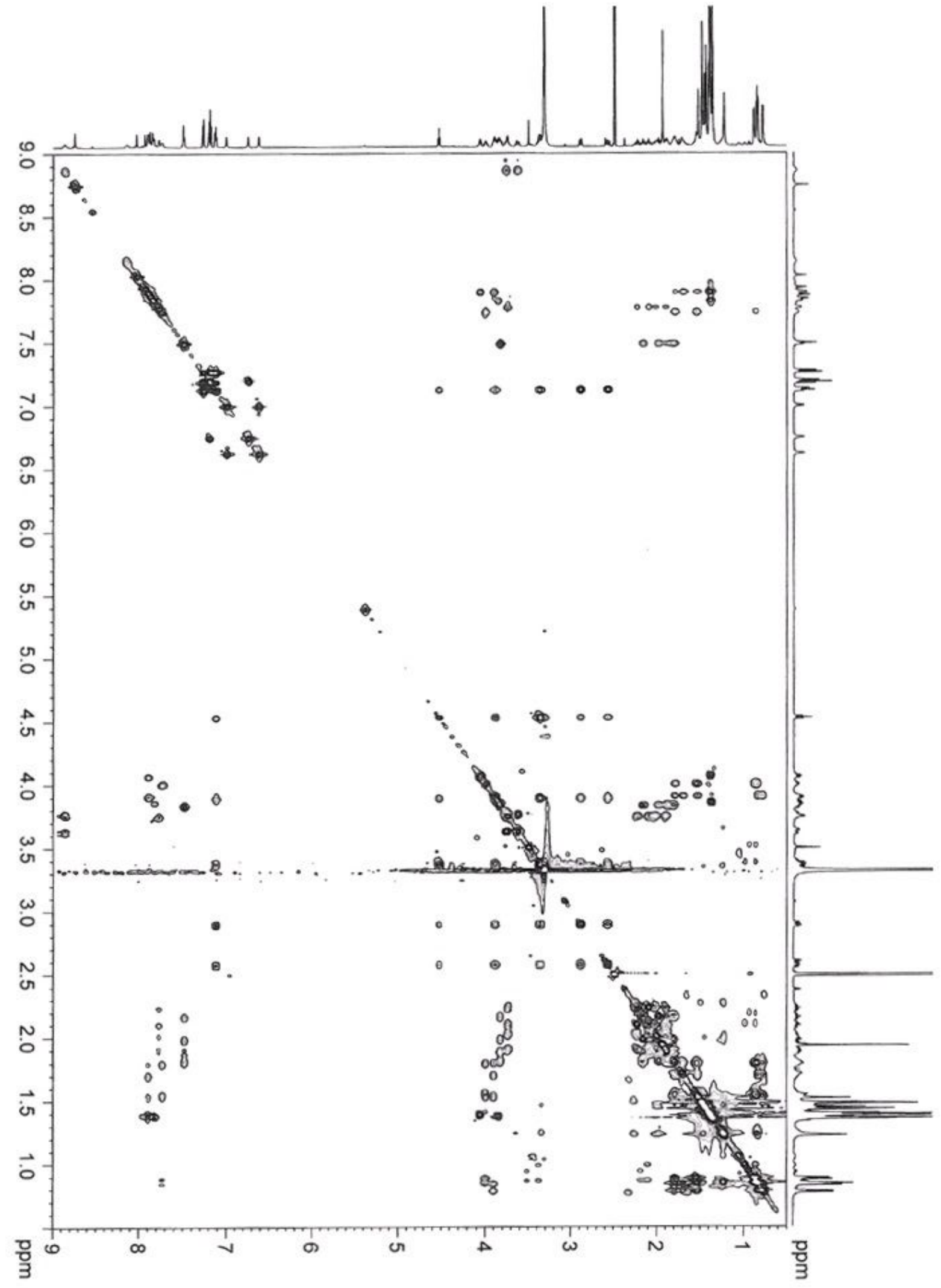


Fig. S23. ${ }^{1} \mathrm{H},{ }^{1} \mathrm{H}$ NOESY spectrum of pentadecaibin II (2) $\left(600 \mathrm{MHz}, \mathrm{DMSO}-d 6,298{ }^{\circ} \mathrm{K}\right)$

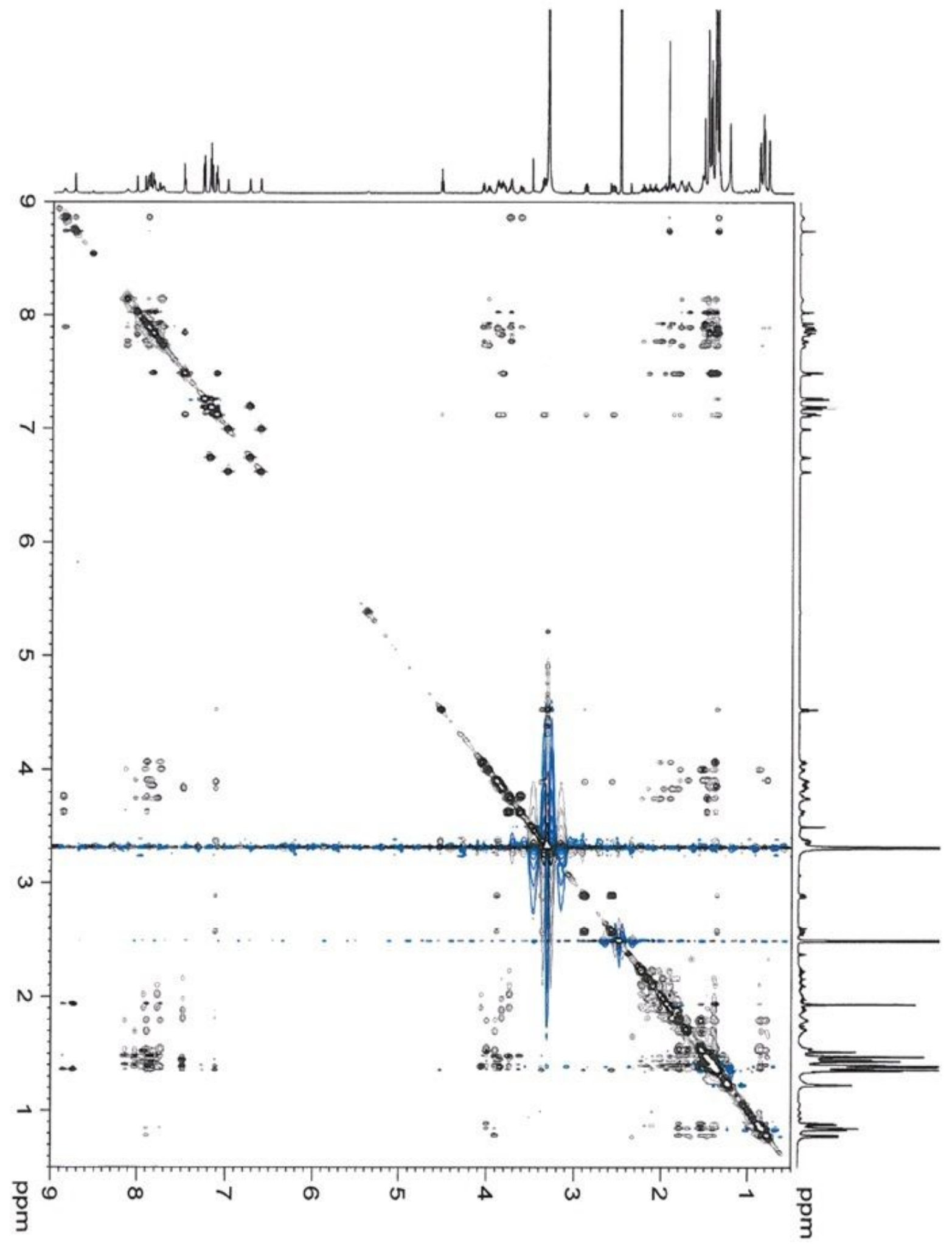


Fig. S24. DEPT-135 spectrum of pentadecaibin II (2) (150 MHz, DMSO- $\left.d 6,298^{\circ} \mathrm{K}\right)$
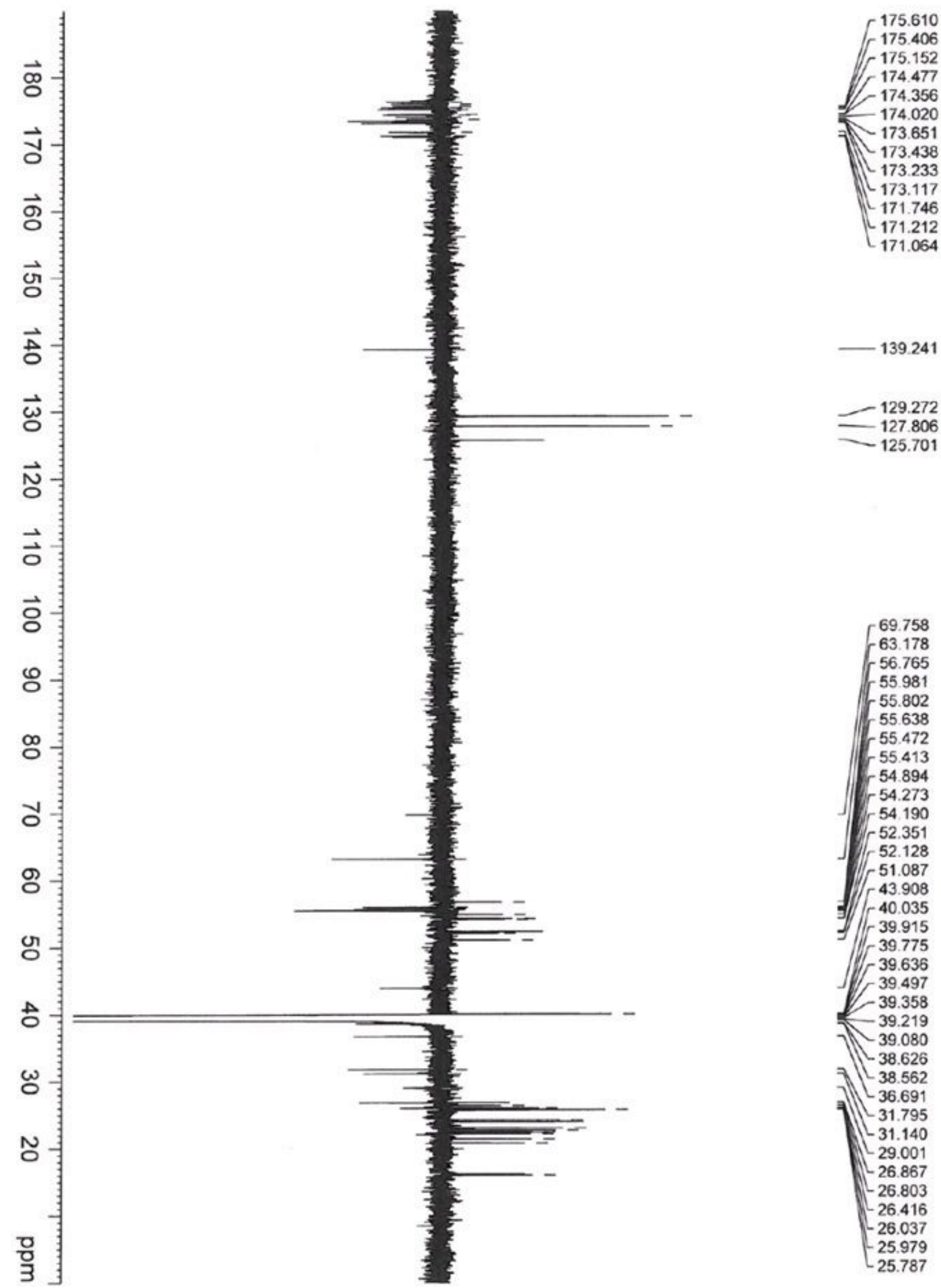

69.758

$-63.178$

56.765

$-55.981$

55.802

55.638

$-55.472$

55.413

$-54.894$

$-54.273$

54.190

$-52.351$

$-52.128$

$-51.087$

$-43.908$

] -40.035

/ 39.915

$-39.775$

$-39.636$

$-39.497$

$-39.358$

$-39.219$

$-39.080$

$-38.626$

$-38.562$

$-36.691$

$-31.795$

$-31.140$

29.001

$-26.867$

$-26.803$

$-26.416$

$-26.037$

25.979

-25.787 
Fig. S25. ${ }^{1} \mathrm{H},{ }^{13} \mathrm{C}$ HSQC spectrum of pentadecaibin II (2) $\left(600 \mathrm{MHz}, \mathrm{DMSO}-d 6,298{ }^{\circ} \mathrm{K}\right)$

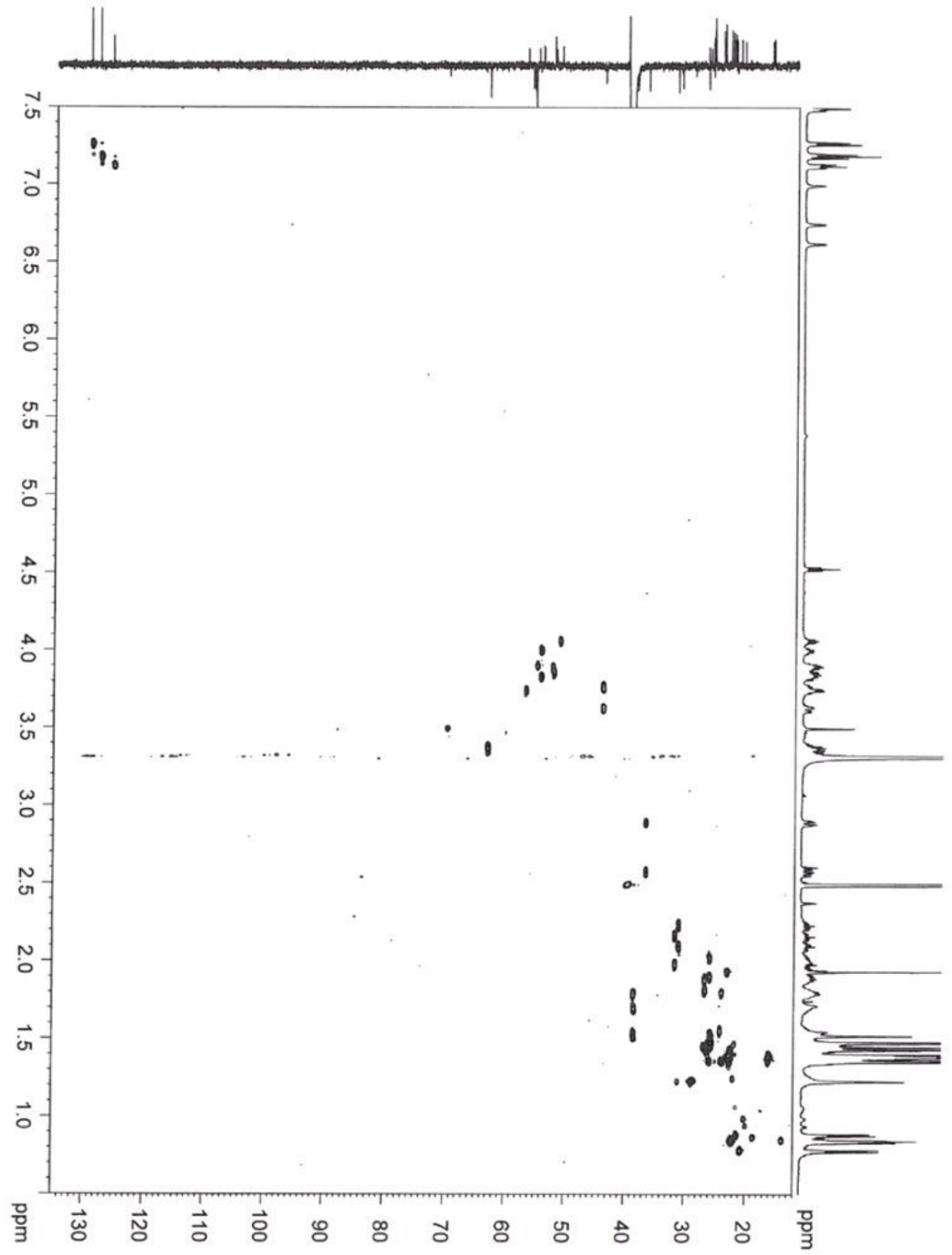


Fig. S26. ${ }^{1} \mathrm{H},{ }^{13} \mathrm{C}$ HMBC spectrum of pentadecaibin II (2) $\left(600 \mathrm{MHz}, \mathrm{DMSO}-d 6,298{ }^{\circ} \mathrm{K}\right)$

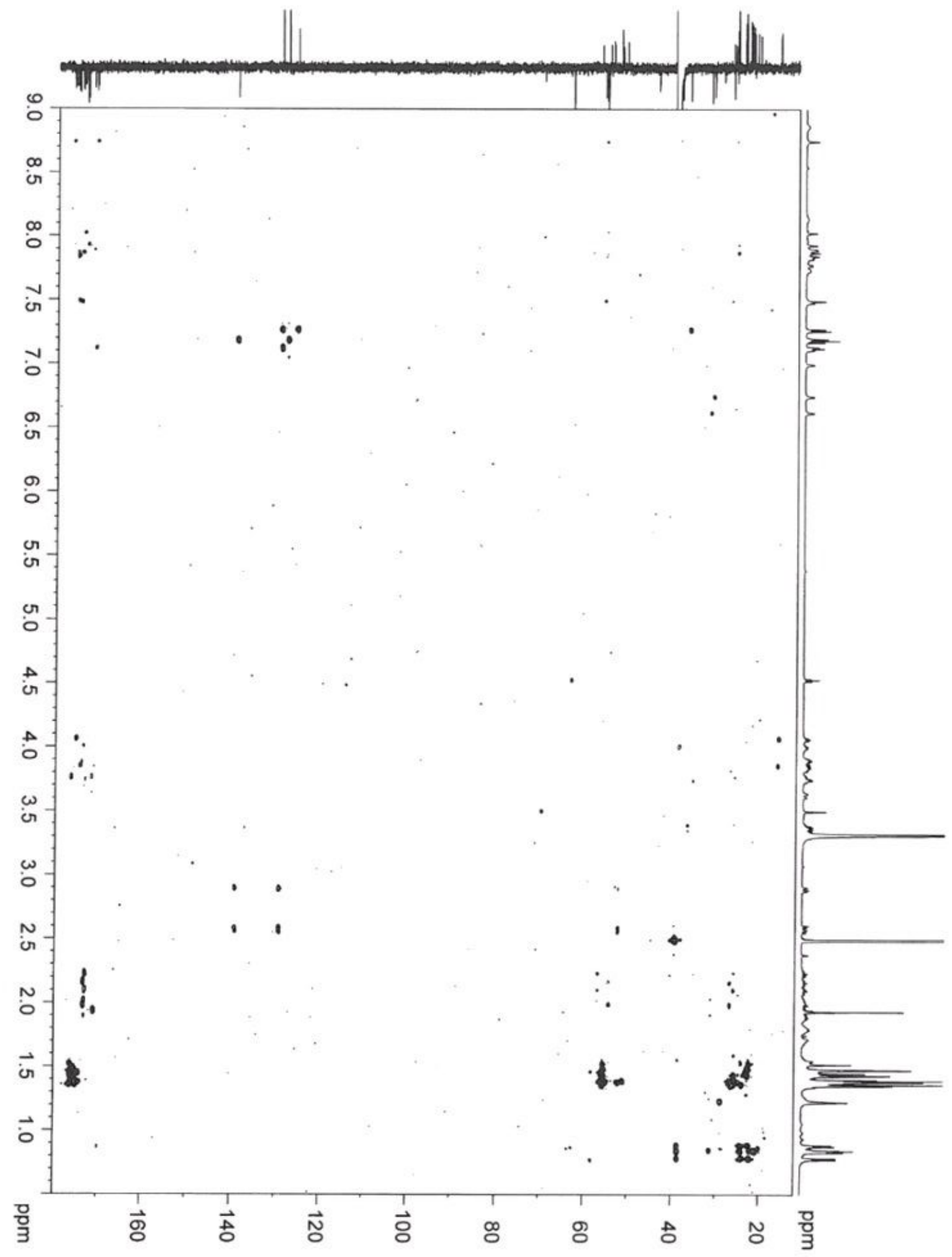


Table S3. ${ }^{1} \mathrm{H}$ and ${ }^{13} \mathrm{C}$ NMR of pentadecaibin II (2) $\left({ }^{1} \mathrm{H} 600 \mathrm{MHz},{ }^{13} \mathrm{C} 150 \mathrm{MHz}\right.$ DMSO-d6, $298^{\circ} \mathrm{K}$ )

\begin{tabular}{|c|c|c|c|c|c|c|c|c|}
\hline $\begin{array}{l}\text { Pos. } \\
\text { Ac-Aib }^{1}\end{array}$ & $\delta_{\mathrm{C}}$, type & $\delta_{\mathrm{H}}$, mult. $(J$ in $\mathrm{Hz})$ & $\begin{array}{l}\text { Pos. } \\
\text { Gln }^{6}\end{array}$ & $\delta_{\mathrm{C}}$, type & $\delta_{\mathrm{H}}$, mult. $(J$ in $\mathrm{Hz})$ & $\begin{array}{l}\text { Pos. } \\
\text { Aib }^{11}\end{array}$ & $\delta_{c}$, type & $\delta_{\mathrm{H}}$, mult. $(J$ in $\mathrm{Hz})$ \\
\hline $\mathrm{C}=\mathrm{O}$ & 176.3, C & - & $\mathrm{C}=\mathrm{O}$ & $173.2, \mathrm{C}$ & - & $\mathrm{C}=\mathrm{O}$ & 175.2, C & - \\
\hline $\mathrm{N}-\mathrm{H}$ & - & $8.74, \mathrm{~s}$ & $\mathrm{~N}-\mathrm{H}$ & - & $7.77, \mathrm{~d}(4.2)$ & $\mathrm{N}-\mathrm{H}$ & - & $7.84, \mathrm{~s}$ \\
\hline$\alpha \mathrm{C}$ & $55.6, \mathrm{C}$ & - & $\alpha \mathrm{CH}$ & $56.8, \mathrm{CH}$ & $3.74, \mathrm{~m}$ & $\alpha \mathrm{C}$ & $55.5, \mathrm{C}$ & - \\
\hline$\beta \mathrm{CH}_{3}$ & $(*), \mathrm{CH}_{3}$ & $\left({ }^{\circ}\right) \mathrm{s}$ & $\beta \mathrm{CH}_{2}$ & $26.0, \mathrm{CH}_{2}$ & 2.02, ddd $(15.2 / 8.5 / 6.7)$ & $\beta \mathrm{CH}_{3}$ & $(*), \mathrm{CH}_{3}$ & $\left({ }^{\circ}\right) \mathrm{s}$ \\
\hline$\beta^{\prime} \mathrm{CH}_{3}$ & $(*), \mathrm{CH}_{3}$ & $\left({ }^{\circ}\right) \mathrm{s}$ & & - & $1.90, \mathrm{~m}$ & $\beta^{\prime} \mathrm{CH}_{3}$ & $(*), \mathrm{CH}_{3}$ & $\left({ }^{\circ}\right) \mathrm{s}$ \\
\hline$\underline{\mathrm{COCH}}_{3}$ & $171, \mathrm{C}$ & - & $\gamma \mathrm{CH}_{2}$ & $31.1, \mathrm{CH}_{2}$ & 2.28, ddd $(15.1,8.7,6.7)$ & & & \\
\hline \multirow[t]{4}{*}{$\mathrm{COCH}_{3}$} & $23.1, \mathrm{CH}_{3}$ & $1.93, \mathrm{~s}$ & & - & 2.09, ddd $(15.1,8.5,6.2)$ & & & \\
\hline & & & $\delta \mathrm{C}=\mathrm{O}$ & $173.1, \mathrm{C}$ & - & & & \\
\hline & & & $\varepsilon \mathrm{NH}_{2 \alpha \mathrm{n}}$ & & $7.19, \mathrm{~s}$ & & & \\
\hline & & & $\varepsilon \mathrm{NH}_{2}$ sy & & $6.74, \mathrm{~s}$ & & & \\
\hline $\mathrm{Gly}^{2}$ & & & $\mathrm{Aib}^{7}$ & & & $\mathrm{Aib}^{12}$ & & \\
\hline $\mathrm{C}=\mathrm{O}$ & $171.7, \mathrm{C}$ & - & $\mathrm{C}=\mathrm{O}$ & $176.3, \mathrm{C}$ & & $\mathrm{C}=\mathrm{O}$ & $174.4, \mathrm{C}$ & - \\
\hline $\mathrm{N}-\mathrm{H}$ & - & 8.86, brs & $\mathrm{N}-\mathrm{H}$ & - & $7.93, \mathrm{~s}$ & $\mathrm{~N}-\mathrm{H}$ & - & $7.49, \mathrm{~s}$ \\
\hline \multirow{3}{*}{$\alpha \mathrm{CH}_{2}$} & $43.9, \mathrm{CH}_{2}$ & 3.75 , dd $(16.3,6.2)$ (pro $S)$ & $\alpha \mathrm{C}$ & $55.6, \mathrm{C}$ & & $\alpha \mathrm{C}$ & $56, \mathrm{C}$ & \\
\hline & - & $3.62, \mathrm{dd}(16.3,4.7)$ (pro R) & $\beta \mathrm{CH}_{3}$ & $(*), \mathrm{CH}_{3}$ & $\left({ }^{\circ}\right) \mathrm{s}$ & $\beta \mathrm{CH}_{3}$ & $(*), \mathrm{CH}_{3}$ & $\left({ }^{\circ}\right) \mathrm{s}$ \\
\hline & & & $\beta^{\prime} \mathrm{CH}_{3}$ & $(*), \mathrm{CH}_{3}$ & $\left({ }^{\circ}\right) \mathrm{s}$ & $\beta^{\prime} \mathrm{CH}_{3}$ & $(*), \mathrm{CH}_{3}$ & $\left({ }^{\circ}\right) \mathrm{s}$ \\
\hline $\mathrm{Ala}^{3}$ & & & $\mathrm{Leu}^{8}$ & & & $\mathrm{Aib}^{13}$ & & \\
\hline $\mathrm{C}=\mathrm{O}$ & $175.4, \mathrm{C}$ & - & $\mathrm{C}=\mathrm{O}$ & $174, \mathrm{C}$ & - & $\mathrm{C}=\mathrm{O}$ & $174.5, \mathrm{C}$ & - \\
\hline $\mathrm{N}-\mathrm{H}$ & - & $7.87, \mathrm{~d}(6.2)$ & $\mathrm{N}-\mathrm{H}$ & - & $7.89, \mathrm{~d}(4.8)$ & $\mathrm{N}-\mathrm{H}$ & - & $7.87, \mathrm{~s}$ \\
\hline$\alpha \mathrm{CH}$ & $51.1, \mathrm{CH}$ & $4.06, \mathrm{dq}(6.2,7.4)$ & $\alpha \mathrm{CH}$ & $54.8, \mathrm{CH}$ & $3.90, \mathrm{~m}$ & $\alpha \mathrm{C}$ & $55.8, \mathrm{C}$ & - \\
\hline \multirow[t]{5}{*}{$\beta \mathrm{CH}_{3}$} & $16.0, \mathrm{CH}_{3}$ & $1.38, \mathrm{~d}(7.4)$ & $\beta \mathrm{CH}_{2}$ & $38.5, \mathrm{CH}_{2}$ & $1.70, \mathrm{~m}$ & $\beta \mathrm{CH}_{3}$ & $(*), \mathrm{CH}_{3}$ & $\left({ }^{\circ}\right) \mathrm{s}$ \\
\hline & & & & - & $1.54, \mathrm{~m}$ & $\beta^{\prime} \mathrm{CH}_{3}$ & $(*), \mathrm{CH}_{3}$ & $\left({ }^{\circ}\right) \mathrm{s}$ \\
\hline & & & $\gamma \mathrm{CH}$ & $24, \mathrm{CH}$ & $1.81, \mathrm{~m}$ & & & \\
\hline & & & $\delta \mathrm{CH}_{3}$ & $22.2, \mathrm{CH}_{3}$ & $0.84, d(6.2)$ & & & \\
\hline & & & $\delta^{\prime} \mathrm{CH}_{3}$ & $20.8, \mathrm{CH}_{3}$ & $0.78, \mathrm{~d}(6.5)$ & & & \\
\hline $\mathrm{Leu}^{4}$ & & & $\mathrm{Aib}^{9}$ & & & $\mathrm{Gln}^{14}$ & & \\
\hline $\mathrm{C}=\mathrm{O}$ & $173.4, \mathrm{C}$ & - & $\mathrm{C}=\mathrm{O}$ & $175.6, \mathrm{C}$ & - & $\mathrm{C}=\mathrm{O}$ & $171.2, \mathrm{C}$ & - \\
\hline $\mathrm{N}-\mathrm{H}$ & - & 7.73, brs & $\mathrm{N}-\mathrm{H}$ & - & $8.03, \mathrm{~s}$ & $\mathrm{~N}-\mathrm{H}$ & - & $7.48, \mathrm{~d}(7.2)$ \\
\hline$\alpha \mathrm{CH}$ & $54.2, \mathrm{CH}$ & $4.00, \operatorname{ddd}(7.0,7.0,5.0)$ & $\alpha \mathrm{C}$ & $55.4, \mathrm{C}$ & - & $\alpha \mathrm{CH}$ & $54.3, \mathrm{CH}$ & $3.83, \mathrm{~m}$ \\
\hline \multirow[t]{2}{*}{$\beta \mathrm{CH}_{2}$} & $38.6, \mathrm{CH}_{2}$ & $1.80, \mathrm{~m}$ & $\mathrm{\beta CH}_{3}$ & $(*), \mathrm{CH}_{3}$ & $\left({ }^{\circ}\right) \mathrm{s}$ & $\beta \mathrm{CH}_{2}$ & $26.8, \mathrm{CH}_{2}$ & $1.87, \mathrm{~m}$ \\
\hline & - & $1.55, \mathrm{~m}$ & $\beta^{\prime} \mathrm{CH}_{3}$ & $\left({ }^{*}\right), \mathrm{CH}_{3}$ & $\left({ }^{\circ}\right) \mathrm{s}$ & & - & $1.80, \mathrm{~m}$ \\
\hline$\gamma \mathrm{CH}$ & $24.3, \mathrm{CH}$ & $1.55, \mathrm{~m}$ & & & & $\gamma \mathrm{CH}_{2}$ & $31.8, \mathrm{CH}_{2}$ & $2.16, \operatorname{ddd}(15.2,9.0,6.2)$ \\
\hline$\delta \mathrm{CH}_{3}$ & $22.4, \mathrm{CH}_{3}$ & $0.83, \mathrm{~d}(6.4)$ & & & & & - & $1.98, \operatorname{ddd}(15.2,8.5,6.7)$ \\
\hline \multirow[t]{3}{*}{$\delta^{\prime} \mathrm{CH}_{3}$} & $21.4, \mathrm{CH}_{3}$ & $0.88, \mathrm{~d}(6.2)$ & & & & $\delta \mathrm{C}=\mathrm{O}$ & $173.4, \mathrm{C}$ & - \\
\hline & & & & & & $\varepsilon \mathrm{NH}_{2 \text { anti }}$ & - & $6.99, \mathrm{~s}$ \\
\hline & & & & & & $\varepsilon \mathrm{NH}_{2 \text { syn }}$ & - & $6.62, \mathrm{~s}$ \\
\hline $\mathrm{Aib}^{5}$ & & & $\mathrm{Ala}^{10}$ & & & Pheol $^{15}$ & & \\
\hline $\mathrm{C}=\mathrm{O}$ & $176.0, \mathrm{C}$ & - & $\mathrm{C}=\mathrm{O}$ & $174.4, \mathrm{C}$ & - & $\mathrm{NH}$ & - & $7.12, \mathrm{~m}$ \\
\hline $\mathrm{N}-\mathrm{H}$ & - & $8.14, \mathrm{~s}$ & $\mathrm{~N}-\mathrm{H}$ & - & $7.83, \mathrm{~d}(4.2)$ & $\alpha \mathrm{CH}$ & $52.4, \mathrm{CH}$ & $3.89, \mathrm{~m}$ \\
\hline$\alpha \mathrm{C}$ & $55.5, \mathrm{C}$ & - & $\alpha \mathrm{CH}$ & $52.1, \mathrm{CH}$ & $3.84, \mathrm{dq}(6.5,7.2)$ & $\beta \mathrm{CH}_{2}$ & $36.7, \mathrm{CH}_{2}$ & $2.89, \mathrm{dd}(13.5,4.5)$ \\
\hline $\mathrm{\beta CH}_{3}$ & $\left({ }^{*}\right), \mathrm{CH}_{3}$ & $\left({ }^{\circ}\right) \mathrm{s}$ & $\mathrm{\beta CH}_{3}$ & $16.2, \mathrm{CH}_{3}$ & $1.37, \mathrm{~d}(7.2)$ & & - & $2.57, \mathrm{dd}(13.5,9.2)$ \\
\hline \multirow[t]{7}{*}{$\beta^{\prime} \mathrm{CH}_{3}$} & $\left({ }^{*}\right), \mathrm{CH}_{3}$ & $\left({ }^{\circ}\right) \mathrm{s}$ & & & & $\beta \mathrm{CH}_{2} \mathrm{OH}$ & $63.2, \mathrm{CH}_{2}$ & $3.37, \mathrm{~m}$ \\
\hline & & & & & & & - & $3.37, \mathrm{~m}$ \\
\hline & & & & & & $\mathrm{OH}$ & - & $4.53, \mathrm{dd}(6.2,6.2)$ \\
\hline & & & & & & $\mathrm{C}-1$ & $139.2, \mathrm{C}$ & \\
\hline & & & & & & $\mathrm{C}-2,6$ & $129.3, \mathrm{CH}$ & $7.27, \mathrm{~m}(7.5)$ \\
\hline & & & & & & $\mathrm{C}-3,5$ & $127.8, \mathrm{CH}$ & $7.18, \mathrm{~m}(7.5,7.5)$ \\
\hline & & & & & & $\mathrm{C}-4$ & $125.7, \mathrm{CH}$ & $7.12, \mathrm{~m}(7.5,7.5)$ \\
\hline \multicolumn{9}{|c|}{$\beta / \beta^{\prime} \mathrm{CH}_{3} \mathrm{Aib}^{1}, \mathrm{Aib}^{5}, \mathrm{Aib}^{7}, \mathrm{Aib}^{9}, \mathrm{Aib}^{11}, \mathrm{Aib}^{12}, \mathrm{Aib}^{13}$. } \\
\hline \multicolumn{9}{|c|}{${ }^{13} \mathrm{C}(*){ }^{2}$} \\
\hline
\end{tabular}

Fig. S27. NMR key correlations of pentadecaibin II (2) (COSY/TOCSY, HMBC and NOESY).

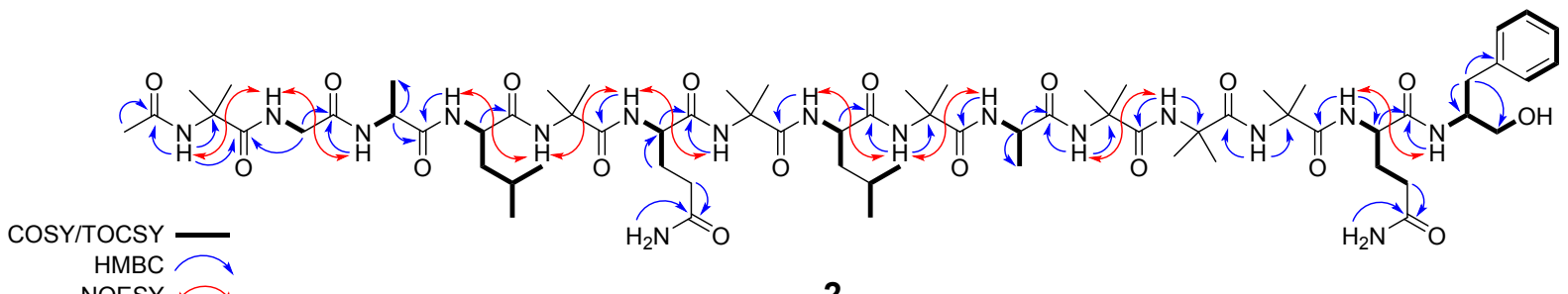


Fig. S28. CD spectrum of pentadecaibin II (2) in $\mathrm{MeOH}$

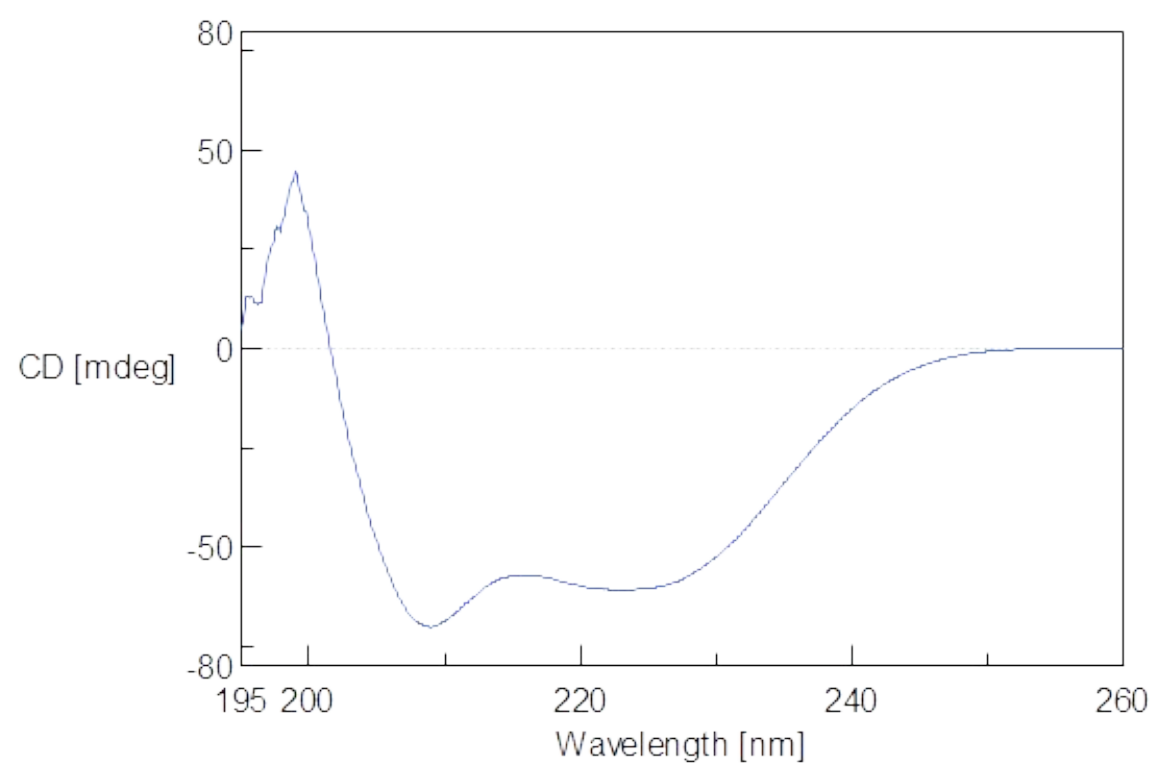


Fig. S29. ${ }^{1} \mathrm{H}$ NMR spectrum of pentadecaibin III (3) $\left(600 \mathrm{MHz}, \mathrm{DMSO}-d 6,298{ }^{\circ} \mathrm{K}\right)$

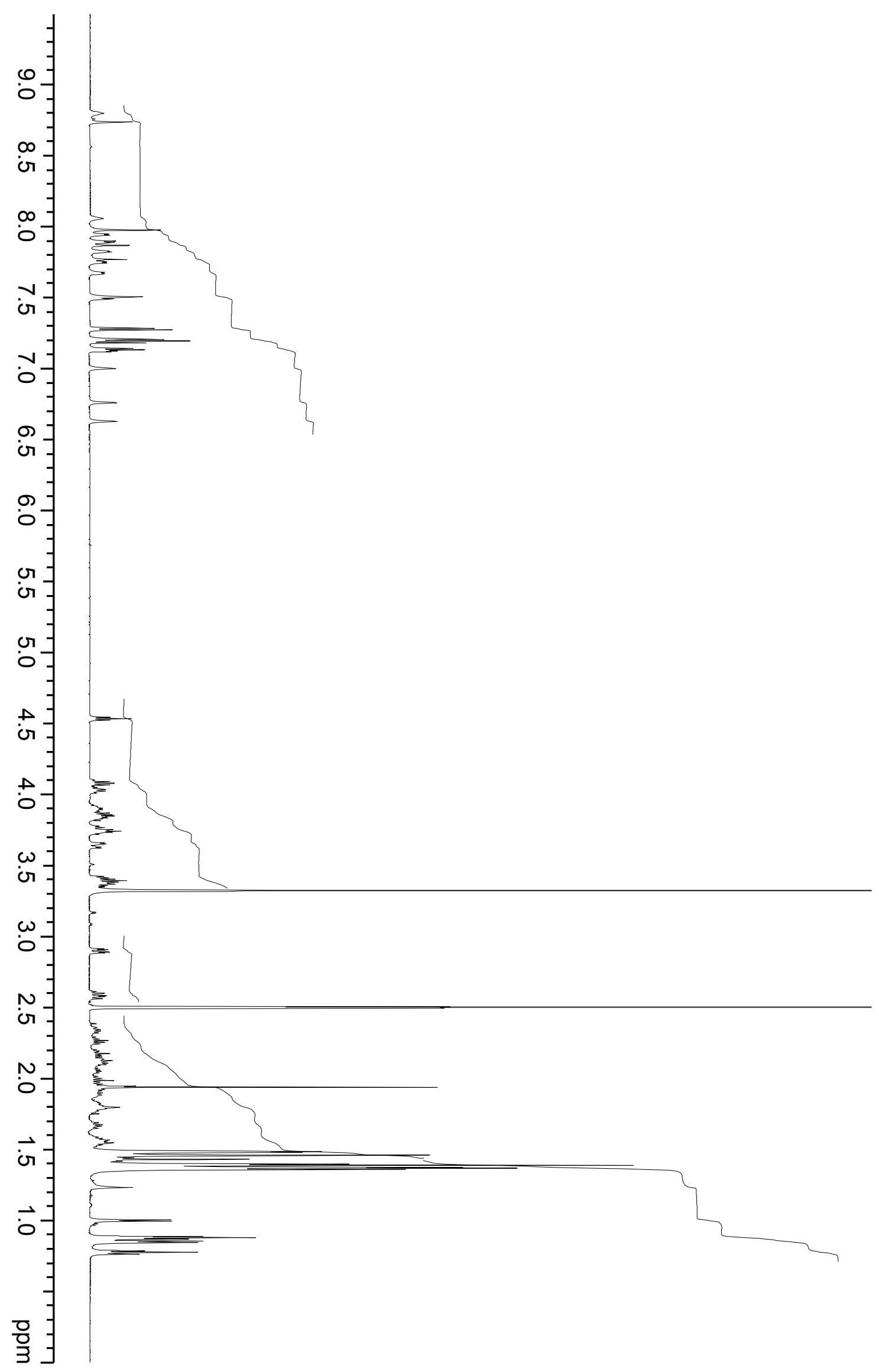


Fig. S30. ${ }^{1} \mathrm{H},{ }^{1} \mathrm{H}$ COSY spectrum of pentadecaibin III (3) $\left(600 \mathrm{MHz}, \mathrm{DMSO}-d 6,298{ }^{\circ} \mathrm{K}\right)$

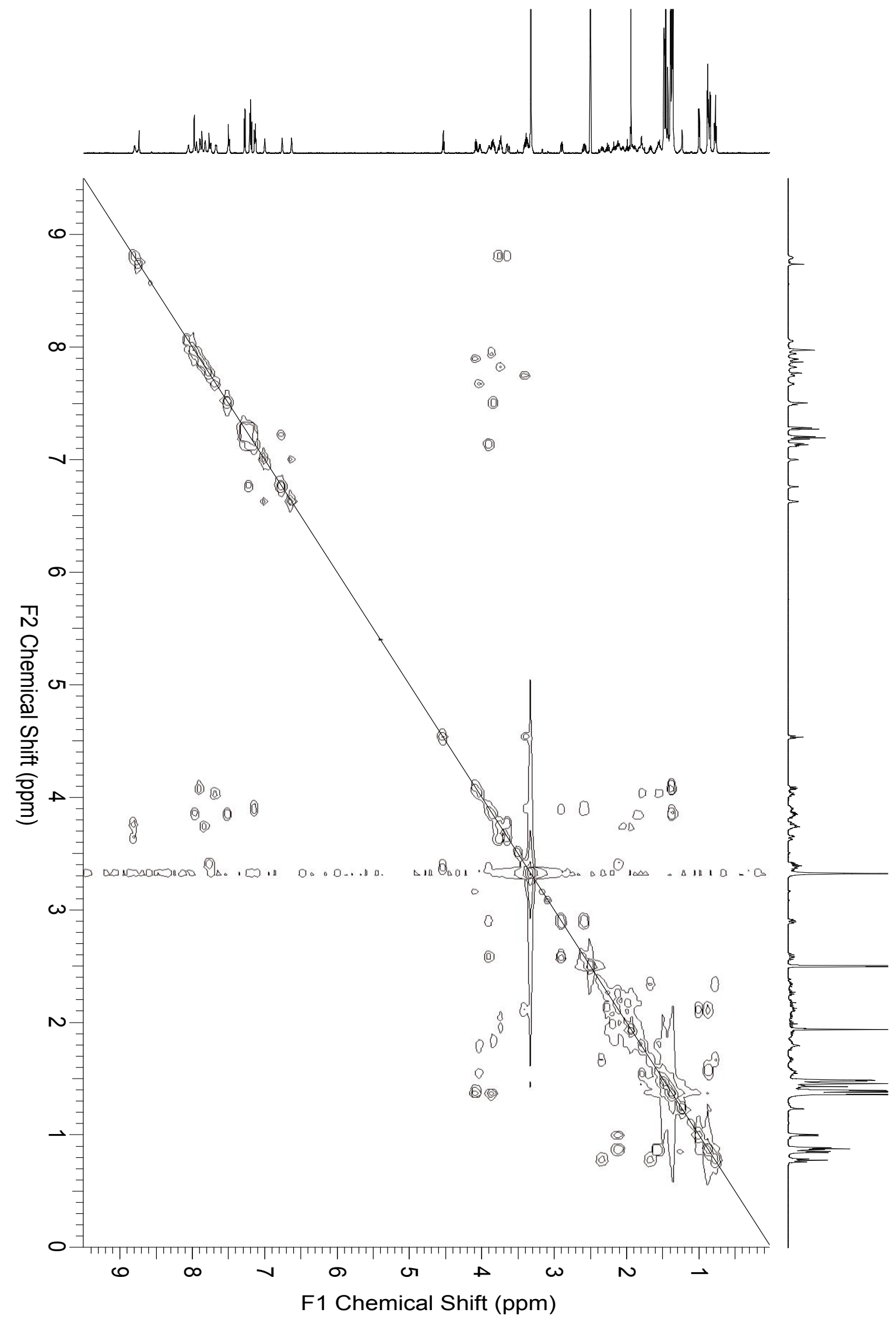


Fig. S31. ${ }^{1} \mathrm{H},{ }^{1} \mathrm{H}$ TOCSY spectrum of pentadecaibin III (3) $\left(600 \mathrm{MHz}, \mathrm{DMSO}-d 6,298{ }^{\circ} \mathrm{K}\right)$

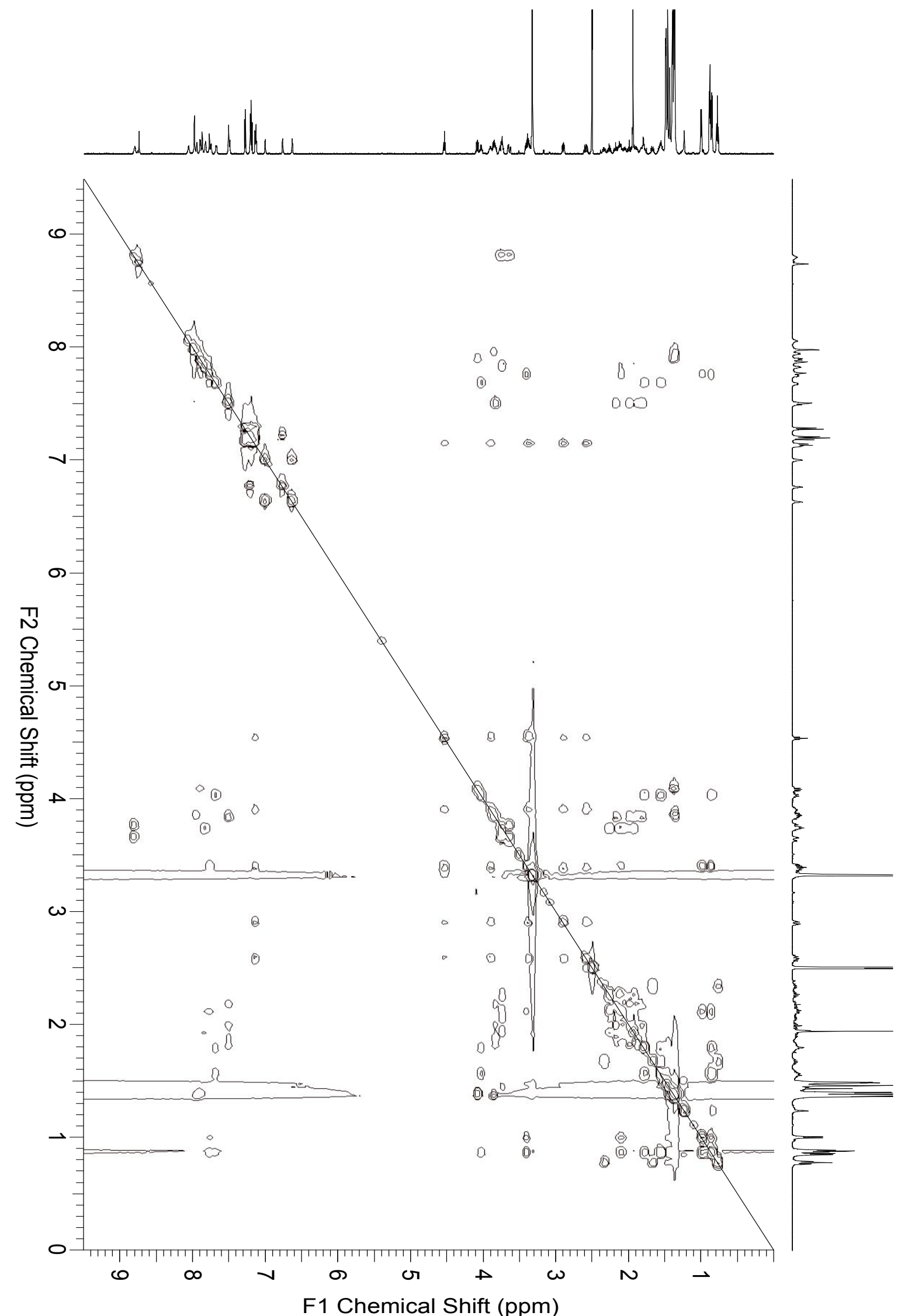


Fig. S32. ${ }^{1} \mathrm{H},{ }^{1} \mathrm{H}$ NOESY spectrum of pentadecaibin III (3) $\left(600 \mathrm{MHz}, \mathrm{DMSO}-d 6,298{ }^{\circ} \mathrm{K}\right)$

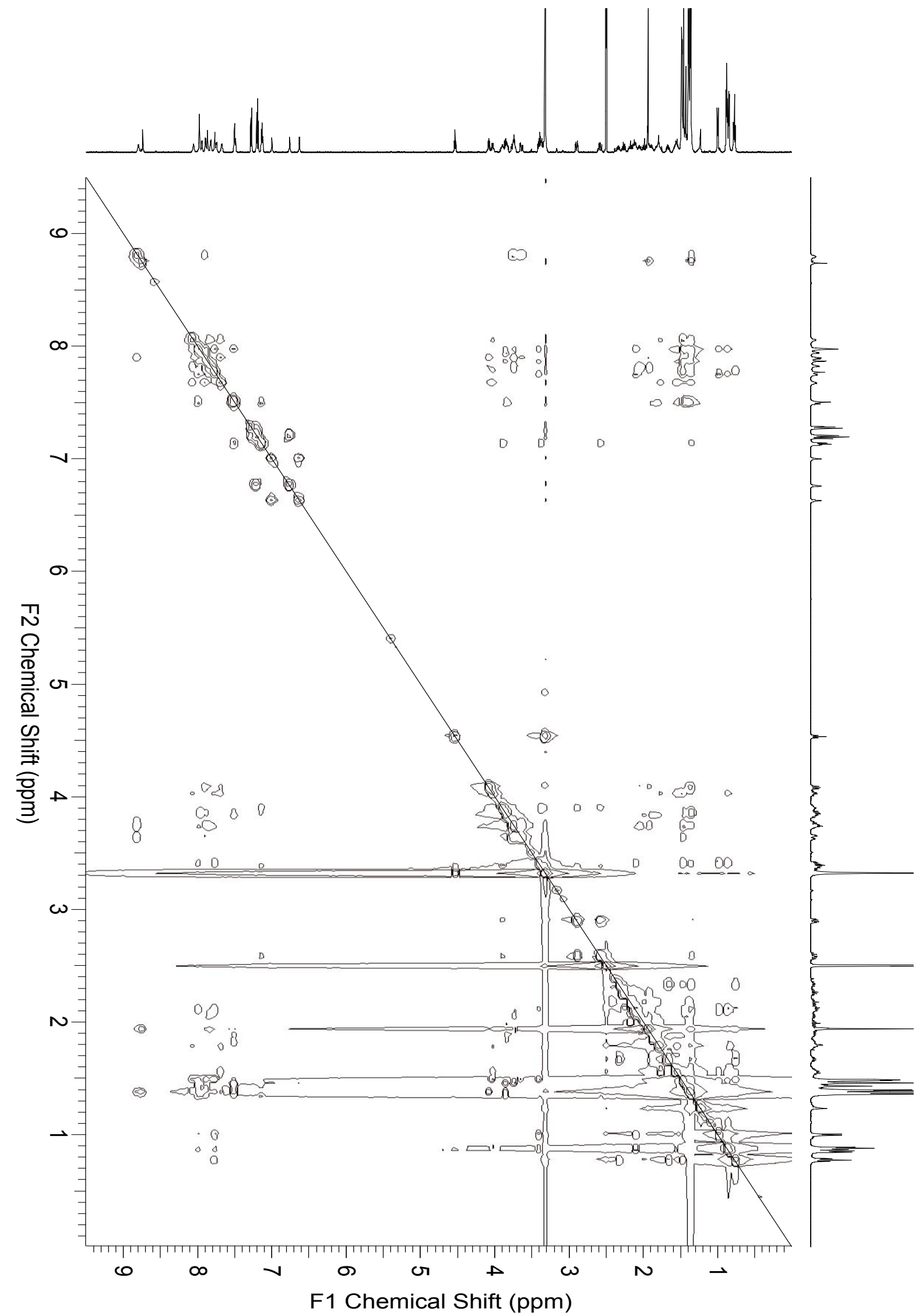


Fig. S33. DEPT-135 spectrum of pentadecaibin III (3) $\left(150 \mathrm{MHz}, \mathrm{DMSO}-d 6,298{ }^{\circ} \mathrm{K}\right)$

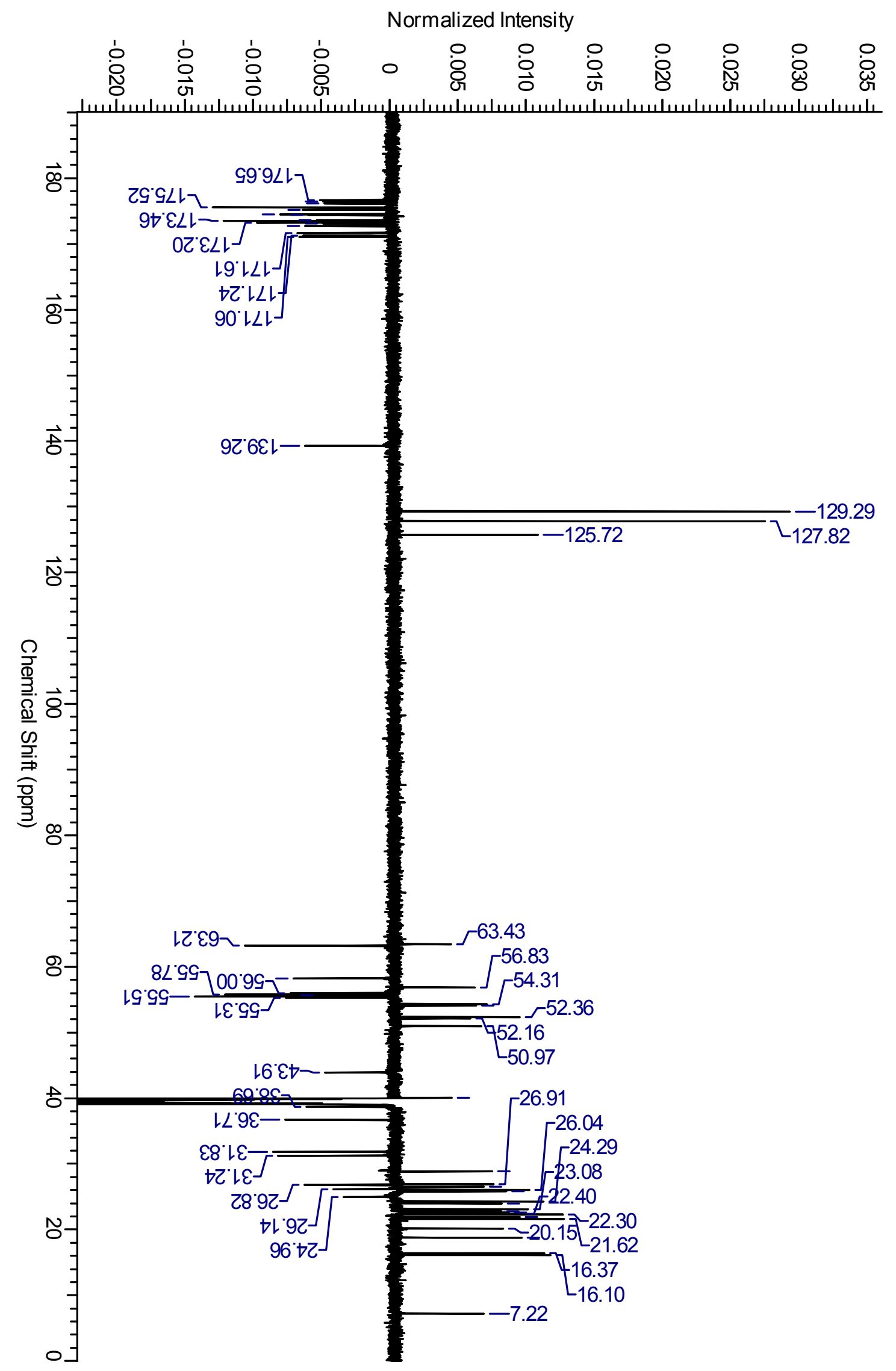


Fig. S34. ${ }^{1} \mathrm{H},{ }^{13} \mathrm{C}$ HSQC spectrum of pentadecaibin III (3) (600 MHz, DMSO-d6, $\left.298{ }^{\circ} \mathrm{K}\right)$

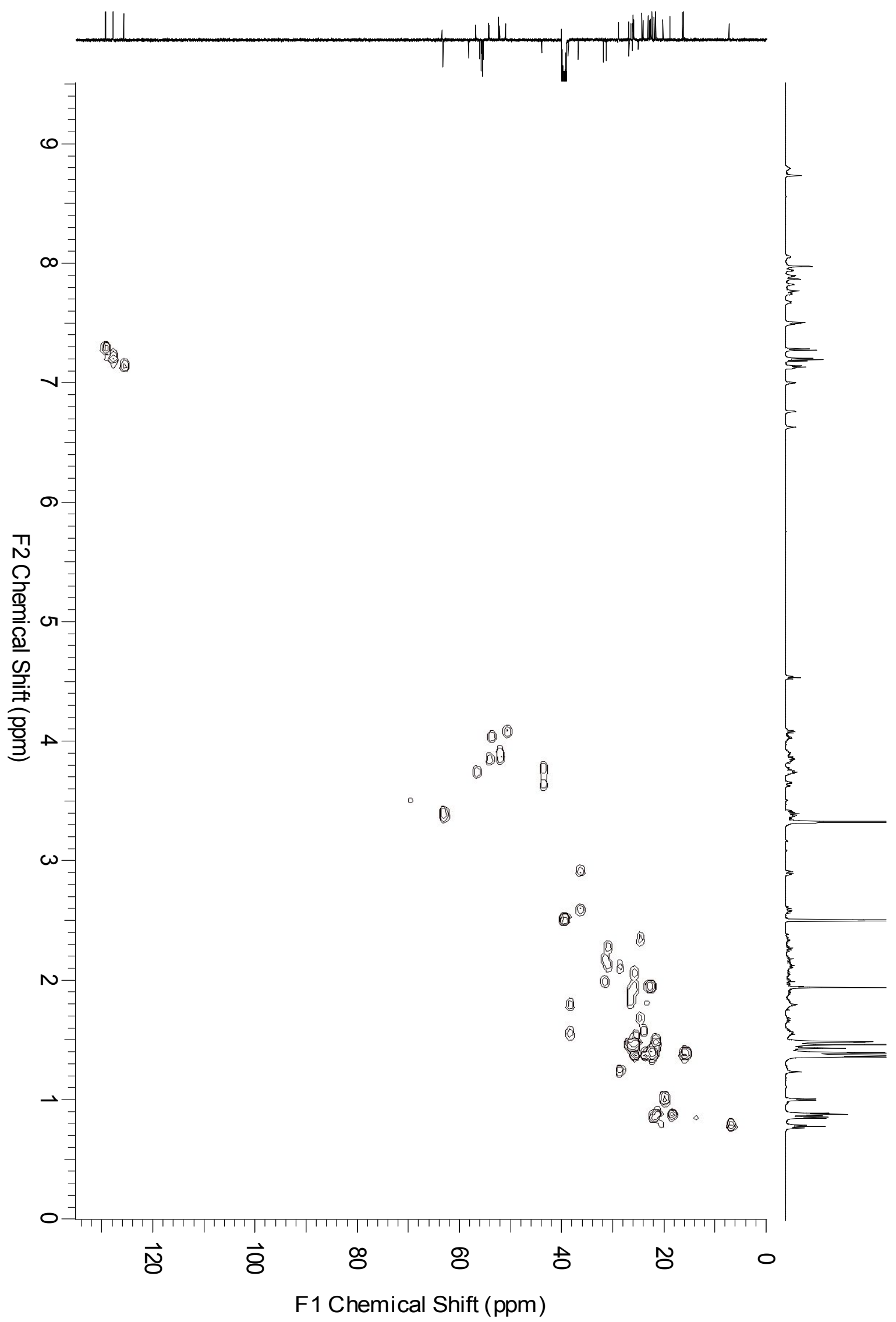


Fig. S35. ${ }^{1} \mathrm{H},{ }^{13} \mathrm{C}$ HMBC spectrum of pentadecaibin III (3) $\left(600 \mathrm{MHz}, \mathrm{DMSO}-d 6,298{ }^{\circ} \mathrm{K}\right)$

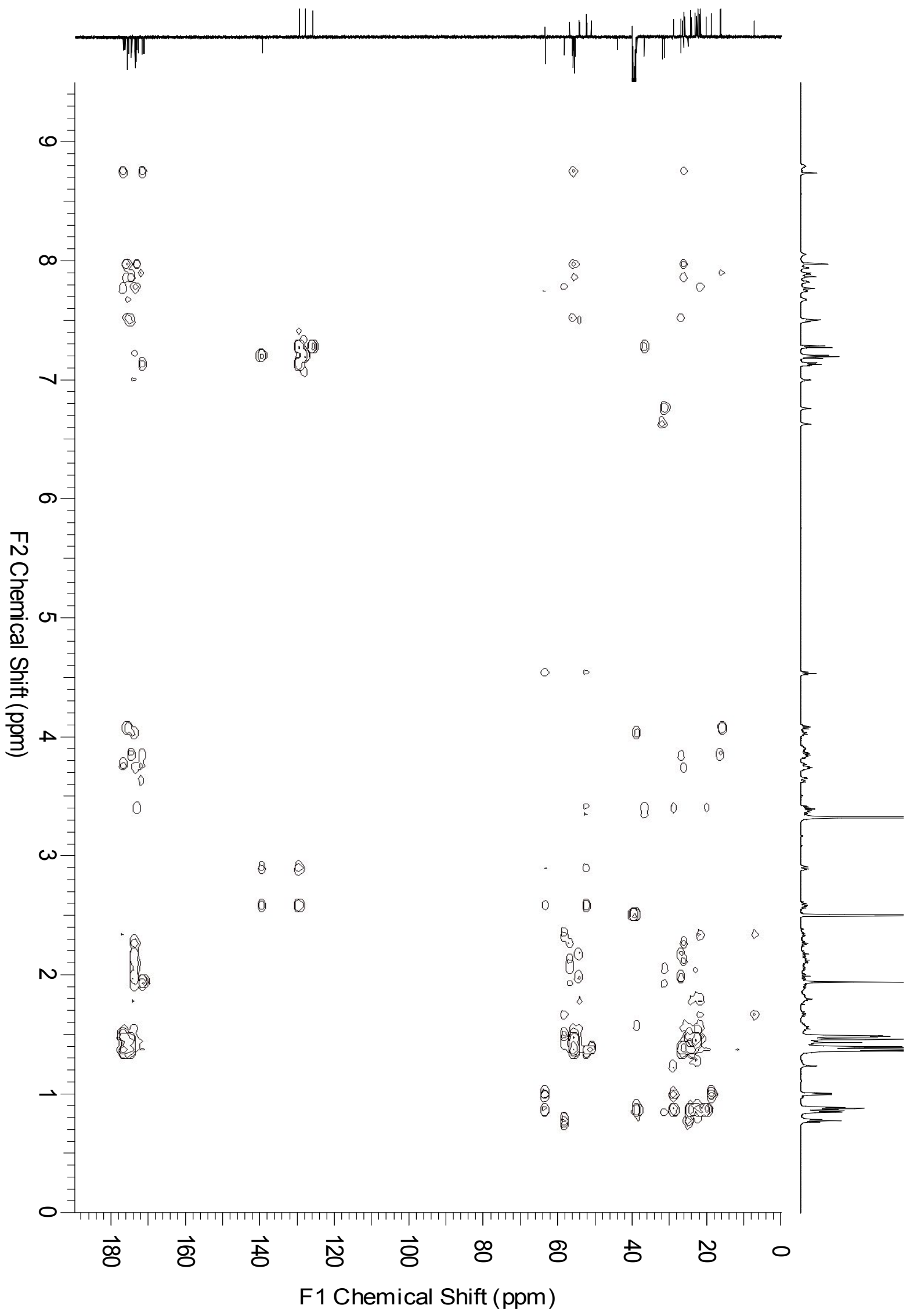


Table S4. ${ }^{1} \mathrm{H}$ and ${ }^{13} \mathrm{C}$ NMR of pentadecaibin III (3) $\left({ }^{1} \mathrm{H} 600 \mathrm{MHz},{ }^{13} \mathrm{C} 150 \mathrm{MHz}\right.$ DMSO-d6, $298^{\circ} \mathrm{K}$ )

\begin{tabular}{|c|c|c|c|c|c|c|c|c|}
\hline $\begin{array}{l}\text { Pos. } \\
\text { Ac-Aib }^{1}\end{array}$ & $\delta_{c}$, type & $\delta_{\mathrm{H}}$, mult. $(J$ in $\mathrm{Hz})$ & $\begin{array}{l}\text { Pos. } \\
\text { Gln }^{6}\end{array}$ & $\delta_{\mathrm{C}}$, type & $\delta_{\mathrm{H}}$, mult. $(J$ in $\mathrm{Hz})$ & $\begin{array}{l}\text { Pos. } \\
\text { Aib }^{11}\end{array}$ & $\delta_{\mathrm{C}}$, type & $\delta_{\mathrm{H}}$, mult. $(J$ in $\mathrm{Hz})$ \\
\hline $\mathrm{C}=\mathrm{O}$ & 176.4, C & - & $\mathrm{C}=\mathrm{O}$ & 173.1, C & - & $\mathrm{C}=\mathrm{O}$ & $175.2, \mathrm{C}$ & - \\
\hline $\mathrm{N}-\mathrm{H}$ & - & $8.73, \mathrm{~s}$ & $\mathrm{~N}-\mathrm{H}$ & - & $7.81, \mathrm{~d}(4.1)$ & $\mathrm{N}-\mathrm{H}$ & - & $7.86, \mathrm{~s}$ \\
\hline$\alpha \mathrm{C}$ & $55.6, \mathrm{C}$ & - & $\alpha \mathrm{CH}$ & $56.8, \mathrm{CH}$ & $3.73, \mathrm{~m}$ & $\alpha \mathrm{C}$ & $55.5, \mathrm{C}$ & - \\
\hline$\beta \mathrm{CH}_{3}$ & $(*), \mathrm{CH}_{3}$ & $\left({ }^{\circ}\right) \mathrm{s}$ & $\beta \mathrm{CH}_{2}$ & $26.1, \mathrm{CH}_{2}$ & $1.89, \mathrm{~m}$ & $\beta \mathrm{CH}_{3}$ & $(*), \mathrm{CH}_{3}$ & $\left({ }^{\circ}\right) \mathrm{s}$ \\
\hline$\beta^{\prime} \mathrm{CH}_{3}$ & $(*), \mathrm{CH}_{3}$ & $\left({ }^{\circ}\right) \mathrm{s}$ & & - & $1.81, \mathrm{~m}$ & $\beta^{\prime} \mathrm{CH}_{3}$ & $(*), \mathrm{CH}_{3}$ & $\left({ }^{\circ}\right) \mathrm{s}$ \\
\hline$\underline{\mathrm{COCH}}_{3}$ & $171, \mathrm{C}$ & - & $\gamma \mathrm{CH}_{2}$ & $31.2, \mathrm{CH}_{2}$ & $2.26, \mathrm{~m}$ & & & \\
\hline \multirow[t]{4}{*}{$\mathrm{COCH}_{3}$} & $23.1, \mathrm{CH}_{3}$ & $1.90, \mathrm{~s}$ & & - & $2.11, \mathrm{~m}$ & & & \\
\hline & & & $\delta \mathrm{C}=\mathrm{O}$ & 173.2, C & - & & & \\
\hline & & & $\varepsilon \mathrm{NH}_{2 \text { an }}$ & & $7.20, \mathrm{~s}$ & & & \\
\hline & & & $\varepsilon \mathrm{NH}_{2}$ sy & & $6.75, \mathrm{~s}$ & & & \\
\hline $\mathrm{Gly}^{2}$ & & & Iva $^{7}$ & & & $\mathrm{Aib}^{12}$ & & \\
\hline $\mathrm{C}=\mathrm{O}$ & 171.6, C & - & $\mathrm{C}=\mathrm{O}$ & 176.6, C & - & $\mathrm{C}=\mathrm{O}$ & $175.5, \mathrm{C}$ & - \\
\hline $\mathrm{N}-\mathrm{H}$ & - & 8.78, brs & $\mathrm{N}-\mathrm{H}$ & - & $7.76, \mathrm{~s}$ & $\mathrm{~N}-\mathrm{H}$ & - & $7.49, \mathrm{~s}$ \\
\hline \multirow[t]{5}{*}{$\alpha \mathrm{CH}_{2}$} & $43.9, \mathrm{CH}_{2}$ & $3.74 \mathrm{dd},(16.2,6.6)$ (pro S) & $\alpha \mathrm{C}$ & $58.1, \mathrm{C}$ & - & $\alpha \mathrm{C}$ & $56, \mathrm{C}$ & - \\
\hline & - & 3.63, dd $(16.2,5.0)$ (pro R) & $\beta \mathrm{CH}_{2}$ & $25.0, \mathrm{CH}_{2}$ & $2.33, \mathrm{~m}$ & $\beta \mathrm{CH}_{3}$ & $(*), \mathrm{CH}_{3}$ & $\left({ }^{\circ}\right) \mathrm{s}$ \\
\hline & & & & - & $1.66, \mathrm{~m}$ & $\beta^{\prime} \mathrm{CH}_{3}$ & $(*), \mathrm{CH}_{3}$ & $\left({ }^{\circ}\right) \mathrm{s}$ \\
\hline & & & $\beta \mathrm{CH}_{3}$ & $21.9, \mathrm{CH}_{3}$ & $1.48, \mathrm{~s}$ & & & \\
\hline & & & $\gamma \mathrm{CH}_{3}$ & $7.2, \mathrm{CH}_{3}$ & 0.76 , dd $(7.4,7.4)$ & & & \\
\hline $\mathrm{Ala}^{3}$ & & & $\mathrm{Val}^{8}$ & & & $\mathrm{Aib}^{13}$ & & \\
\hline $\mathrm{C}=\mathrm{O}$ & 175.2, C & - & $\mathrm{C}=\mathrm{O}$ & 172.7, C & - & $\mathrm{C}=\mathrm{O}$ & 174.5, C & - \\
\hline $\mathrm{N}-\mathrm{H}$ & - & $7.88, \mathrm{~d}(5.9)$ & $\mathrm{N}-\mathrm{H}$ & - & $7.74, d(5.1)$ & $\mathrm{N}-\mathrm{H}$ & - & $7.96, \mathrm{~s}$ \\
\hline$\alpha \mathrm{CH}$ & $51, \mathrm{CH}$ & $4.07, \mathrm{dq}(5.9,7.5)$ & $\alpha \mathrm{CH}$ & $63.4, \mathrm{CH}$ & $3.39, \mathrm{~m}$ & $\alpha \mathrm{C}$ & $55.8, \mathrm{C}$ & \\
\hline \multirow[t]{3}{*}{$\beta \mathrm{CH}_{3}$} & $16.1, \mathrm{CH}_{3}$ & $1.36, \mathrm{~d}(7.5)$ & $\beta \mathrm{CH}$ & $28.8, \mathrm{CH}$ & $2.10, \mathrm{~m}$ & $\beta \mathrm{CH}_{3}$ & (*), $\mathrm{CH}_{3}$ & $\left(^{\circ}\right) \mathrm{s}$ \\
\hline & & & $\gamma \mathrm{CH}_{3}$ & $18.8, \mathrm{CH}_{3}$ & $0.99, \mathrm{~d}(6.4)$ & $\beta^{\prime} \mathrm{CH}_{3}$ & $(*), \mathrm{CH}_{3}$ & $\left({ }^{\circ}\right) \mathrm{s}$ \\
\hline & & & $\gamma^{\prime} \mathrm{CH}_{3}$ & $21.1, \mathrm{CH}_{3}$ & $0.86, d(6.5)$ & & & \\
\hline $\mathrm{Leu}^{4}$ & & & $\mathrm{Aib}^{9}$ & & & $\mathrm{Gln}^{14}$ & & \\
\hline $\mathrm{C}=\mathrm{O}$ & 173.6, C & - & $\mathrm{C}=\mathrm{O}$ & $175.5, \mathrm{C}$ & - & $\mathrm{C}=\mathrm{O}$ & $171.2, \mathrm{C}$ & - \\
\hline $\mathrm{N}-\mathrm{H}$ & - & $7.66, d(6.3)$ & $\mathrm{N}-\mathrm{H}$ & - & $7.96, \mathrm{~s}$ & $\mathrm{~N}-\mathrm{H}$ & - & $7.48, \mathrm{~m}$ \\
\hline$\alpha \mathrm{CH}$ & $54.1, \mathrm{CH}$ & $4.02, \mathrm{~m}$ & $\alpha \mathrm{C}$ & $55.3, \mathrm{C}$ & - & $\alpha \mathrm{CH}$ & $54.3, \mathrm{CH}$ & $3.82, \mathrm{~m}$ \\
\hline \multirow[t]{2}{*}{$\beta \mathrm{CH}_{2}$} & $38.7, \mathrm{CH}_{2}$ & $1.78, \mathrm{~m}$ & $\beta \mathrm{CH}_{3}$ & $(*), \mathrm{CH}_{3}$ & $\left(^{\circ}\right) \mathrm{s}$ & $\beta \mathrm{CH}_{2}$ & $26.8, \mathrm{CH}_{2}$ & $2.04, \mathrm{~m}$ \\
\hline & - & $1.55, \mathrm{~m}$ & $\beta^{\prime} \mathrm{CH}_{3}$ & $(*), \mathrm{CH}_{3}$ & $\left({ }^{\circ}\right) \mathrm{s}$ & & - & $1.92, \mathrm{~m}$ \\
\hline$\gamma \mathrm{CH}$ & $24.3, \mathrm{CH}$ & $1.53, \mathrm{~m}$ & & & & $\gamma \mathrm{CH}_{2}$ & $31.8, \mathrm{CH}_{2}$ & $2.17, \mathrm{~m}$ \\
\hline$\delta \mathrm{CH}_{3}$ & $22.3, \mathrm{CH}_{3}$ & $0.84, \mathrm{~d}(6.3)$ & & & & & - & $1.98, \mathrm{~m}$ \\
\hline \multirow[t]{3}{*}{$\delta^{\prime} \mathrm{CH}_{3}$} & $21.6, \mathrm{CH}_{3}$ & $0.86, \mathrm{~d}(6.5)$ & & & & $\delta \mathrm{C}=\mathrm{O}$ & 173.4, C & - \\
\hline & & & & & & $\varepsilon \mathrm{NH}_{2 \text { anti }}$ & - & $6.99, \mathrm{~s}$ \\
\hline & & & & & & $\varepsilon \mathrm{NH}_{2}$ syn & - & $6.61 \mathrm{~s}$ \\
\hline $\mathrm{Aib}^{5}$ & & & $\mathrm{Ala}^{10}$ & & & Pheol $^{15}$ & & \\
\hline $\mathrm{C}=\mathrm{O}$ & 176.1, C & - & $\mathrm{C}=\mathrm{O}$ & $174.3, \mathrm{C}$ & - & $\mathrm{NH}$ & - & $7.11, \mathrm{~m}$ \\
\hline $\mathrm{N}-\mathrm{H}$ & - & $8.05, \mathrm{~s}$ & $\mathrm{~N}-\mathrm{H}$ & - & $7.93, \mathrm{~d}(4.7)$ & $\alpha \mathrm{CH}$ & $52.4, \mathrm{CH}$ & $3.89, \mathrm{~m}$ \\
\hline$\alpha \mathrm{C}$ & 55.5 & - & $\alpha \mathrm{CH}$ & $52.2, \mathrm{CH}$ & $3.85, \mathrm{~m}$ & $\beta \mathrm{CH}_{2}$ & $36.7, \mathrm{CH}_{2}$ & $2.89, \mathrm{dd}(13.6,4.4)$ \\
\hline$\beta \mathrm{CH}_{3}$ & $(*), \mathrm{CH}_{3}$ & $\left({ }^{\circ}\right) \mathrm{s}$ & $\beta \mathrm{CH}_{3}$ & $16.4, \mathrm{CH}_{3}$ & $1.35, \mathrm{~d}(5.5)$ & & - & $2.57, \mathrm{dd}(13.6,9.1)$ \\
\hline \multirow[t]{7}{*}{$\beta^{\prime} \mathrm{CH}_{3}$} & (*), $\mathrm{CH}_{3}$ & $\left({ }^{\circ}\right) \mathrm{s}$ & & & & $\beta \mathrm{CH}_{2} \mathrm{OH}$ & $63.2, \mathrm{CH}_{2}$ & $3.37, \mathrm{~m}$ \\
\hline & & & & & & & - & $3.37, \mathrm{~m}$ \\
\hline & & & & & & $\mathrm{OH}$ & - & $4.52, \mathrm{dd}(6.1,6.1)$ \\
\hline & & & & & & $\mathrm{C}-1$ & $139.2, \mathrm{C}$ & \\
\hline & & & & & & $\mathrm{C}-2,6$ & $129.3, \mathrm{CH}$ & $7.26, \mathrm{~m}(\mathrm{~d})(8.2)$ \\
\hline & & & & & & $\mathrm{C}-3,5$ & $127.8, \mathrm{CH}$ & $7.14, \mathrm{~m}(\mathrm{t})(8.2)$ \\
\hline & & & & & & $\mathrm{C}-4$ & $125.7, \mathrm{CH}$ & $7.12, \mathrm{~m}(\mathrm{t})(8.2)$ \\
\hline \multicolumn{9}{|c|}{$\beta / \beta^{\prime} \mathrm{CH}_{3} \mathrm{Aib}^{1}, \mathrm{Aib}^{5}, \mathrm{Aib}^{9}, \mathrm{Aib}^{11}, \mathrm{Aib}^{12}, \mathrm{Aib}^{13}$. } \\
\hline & & 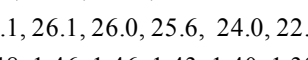 & & & & & & \\
\hline
\end{tabular}

Fig. S36. NMR key correlations of pentadecaibin III (3) (COSY/TOCSY, HMBC and NOESY).

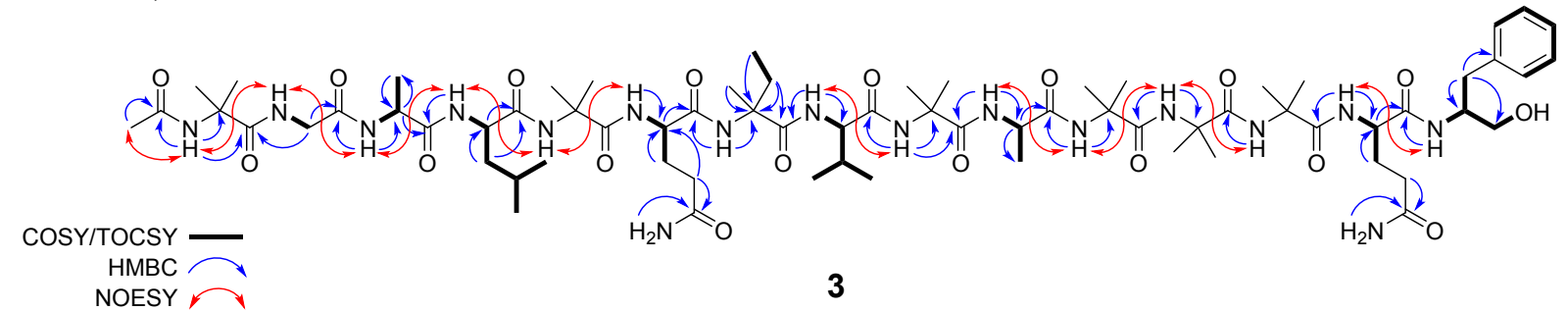


Fig. S37. CD spectrum of pentadecaibin III (3) in $\mathrm{MeOH}$

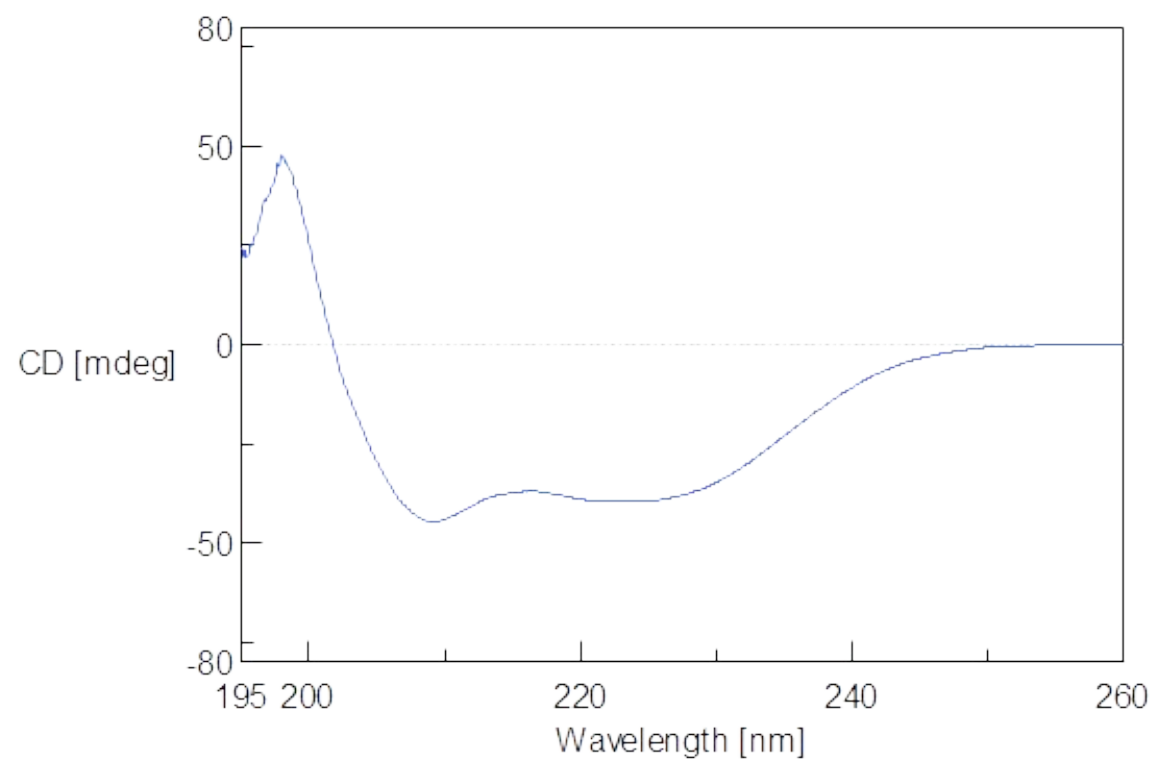


Fig. S38. ${ }^{1} \mathrm{H}$ NMR spectrum of pentadecaibin IV (4) (600 MHz, DMSO- $\left.d 6,298^{\circ} \mathrm{K}\right)$

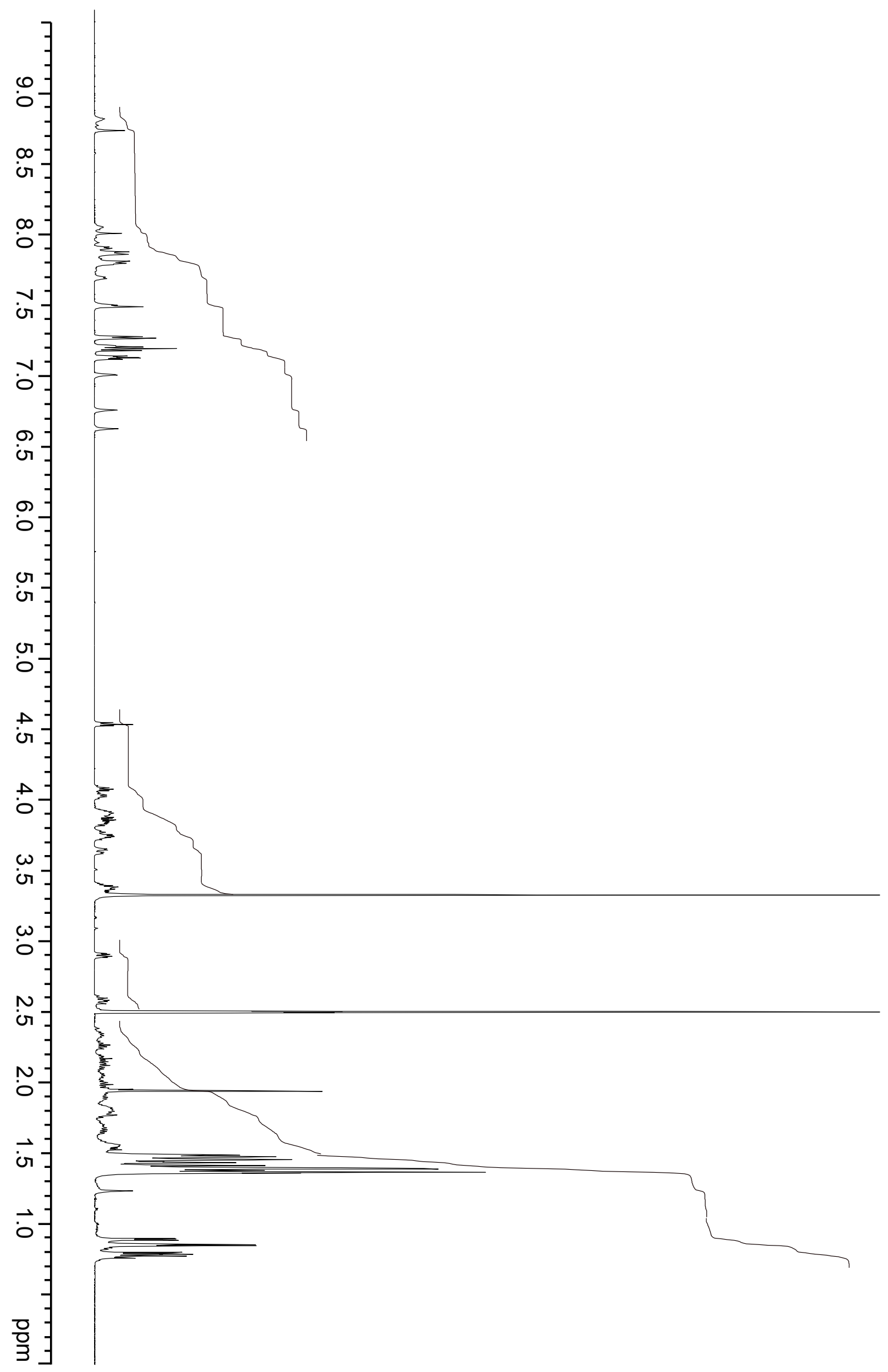


Fig. S39. ${ }^{1} \mathrm{H},{ }^{1} \mathrm{H}$ COSY spectrum of pentadecaibin IV (4) $\left(600 \mathrm{MHz}, \mathrm{DMSO}-d 6,298{ }^{\circ} \mathrm{K}\right)$

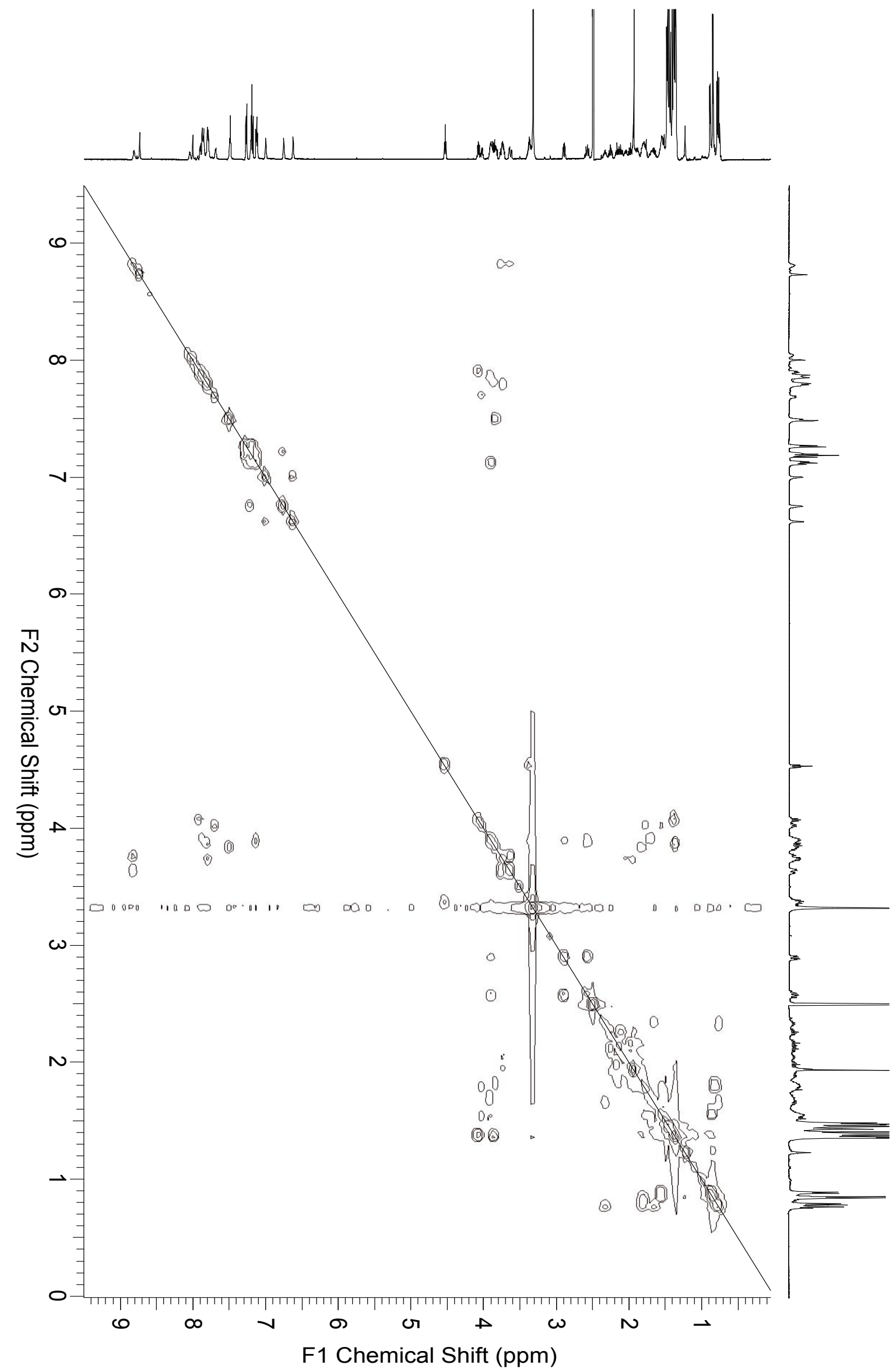


Fig. S40. ${ }^{1} \mathrm{H},{ }^{1} \mathrm{H}$ TOCSY spectrum of pentadecaibin IV (4) $\left(600 \mathrm{MHz}, \mathrm{DMSO}-d 6,298{ }^{\circ} \mathrm{K}\right)$

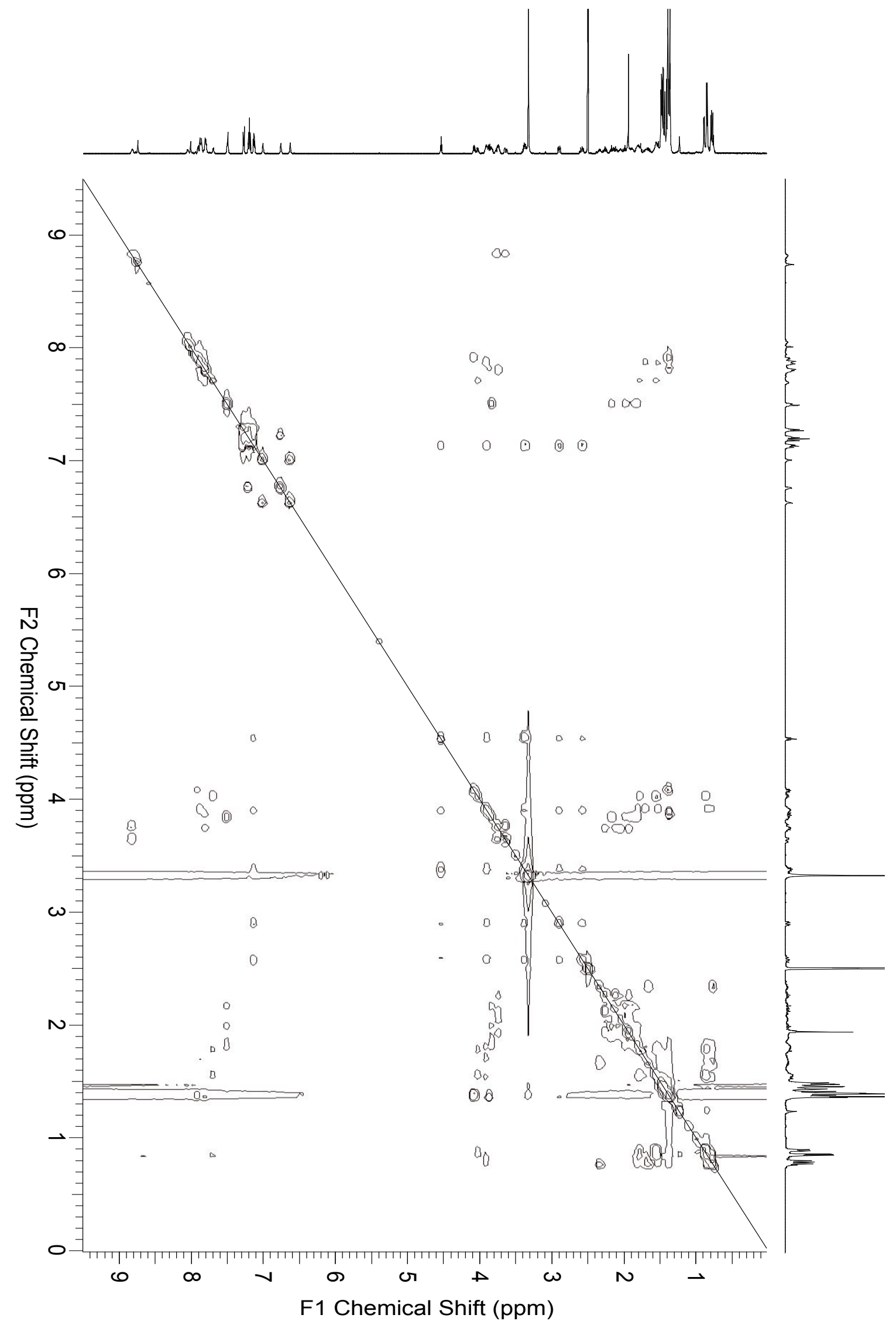


Fig. S41. ${ }^{1} \mathrm{H},{ }^{1} \mathrm{H}$ NOESY spectrum of pentadecaibin IV (4) (600 MHz, DMSO-d6, $\left.298{ }^{\circ} \mathrm{K}\right)$

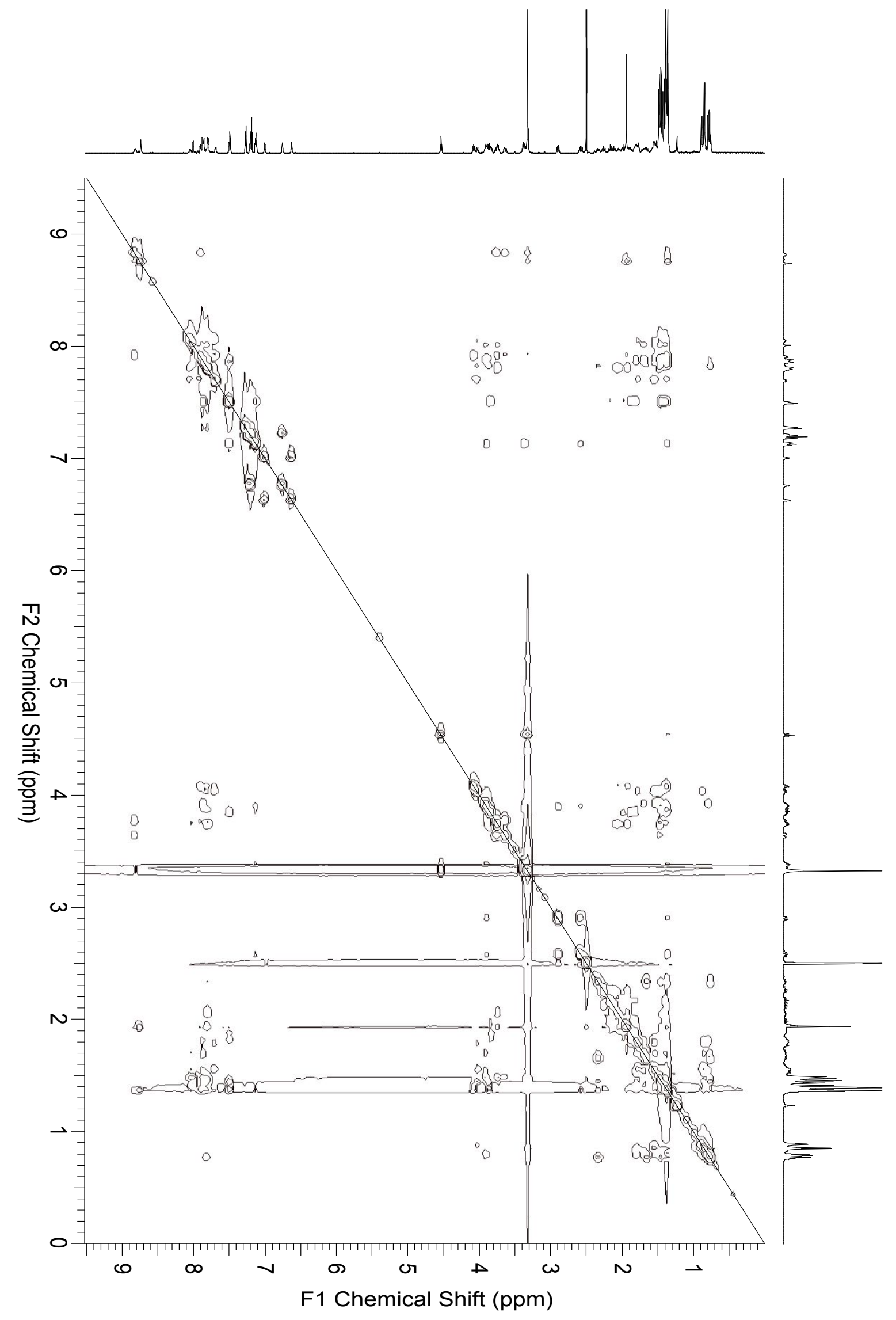


Fig. S42. DEPT-135 spectrum of pentadecaibin IV (4) (150 MHz, DMSO- $\left.d 6,298^{\circ} \mathrm{K}\right)$

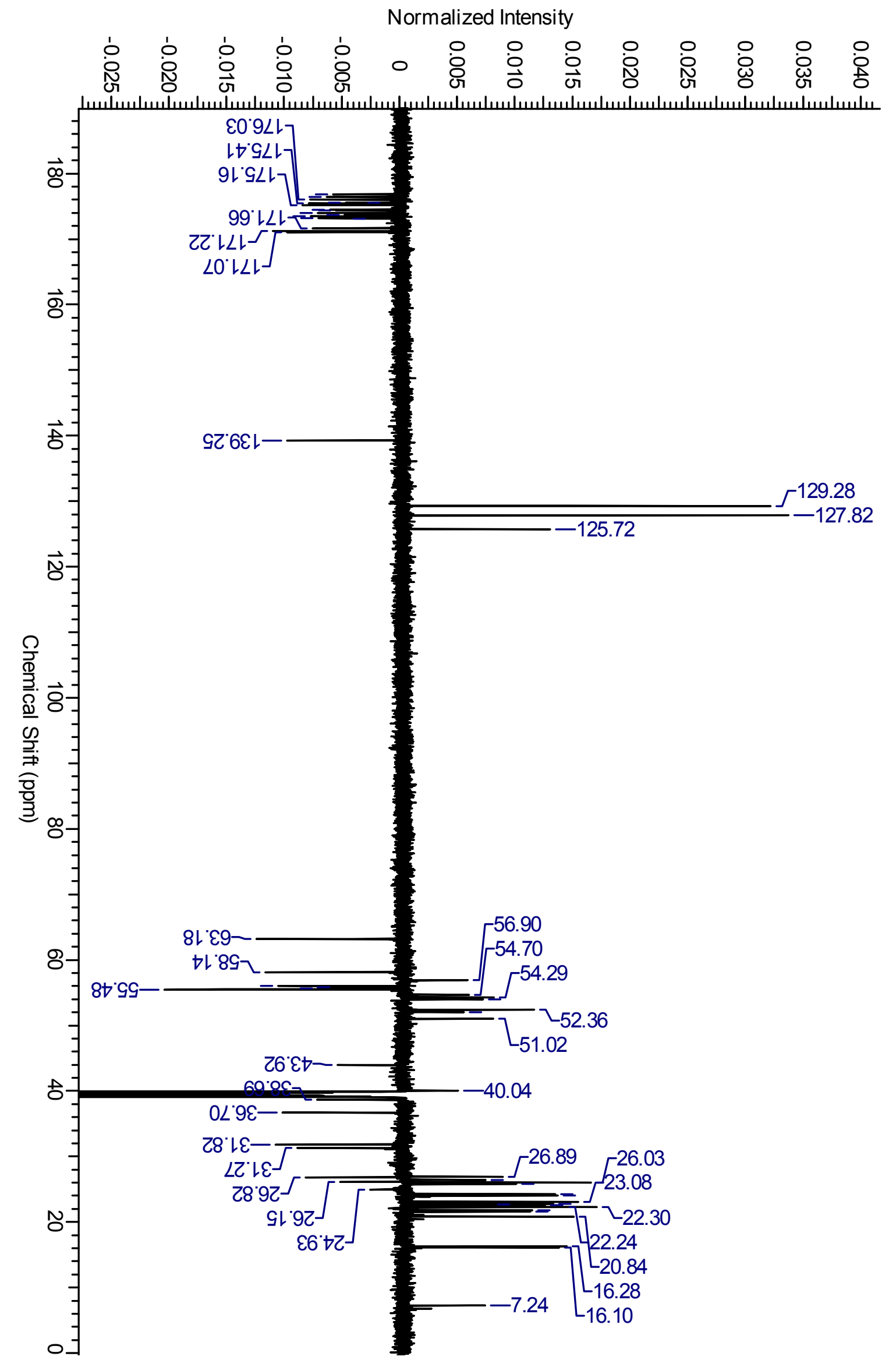


Fig. S43. ${ }^{1} \mathrm{H},{ }^{13} \mathrm{C}$ HSQC spectrum of pentadecaibin IV (4) $\left(600 \mathrm{MHz}\right.$, DMSO-d6, $\left.298{ }^{\circ} \mathrm{K}\right)$

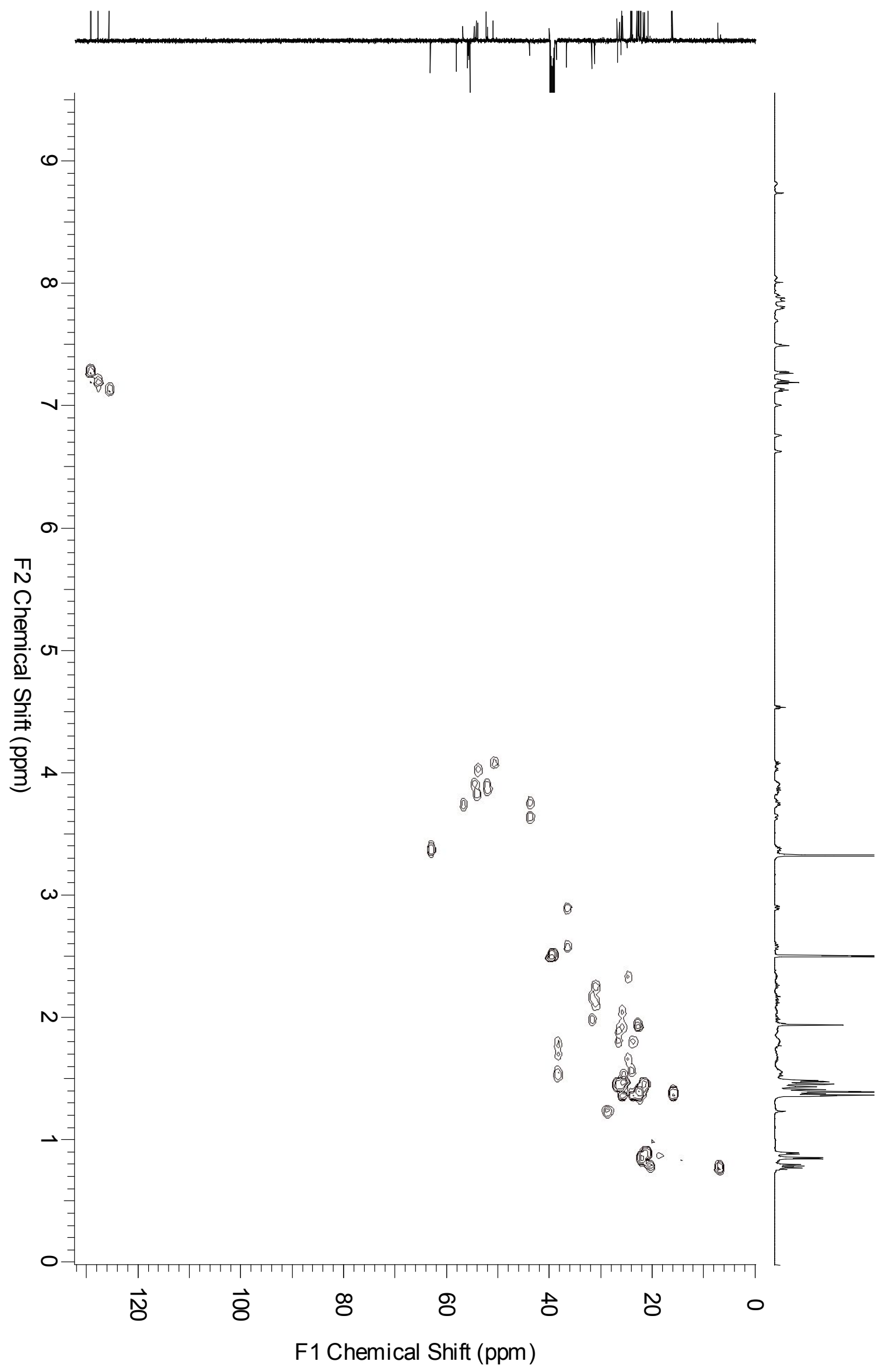


Fig. S44. ${ }^{1} \mathrm{H},{ }^{13} \mathrm{C}$ HMBC spectrum of pentadecaibin IV (4) (600 MHz, DMSO-d6, $\left.298{ }^{\circ} \mathrm{K}\right)$

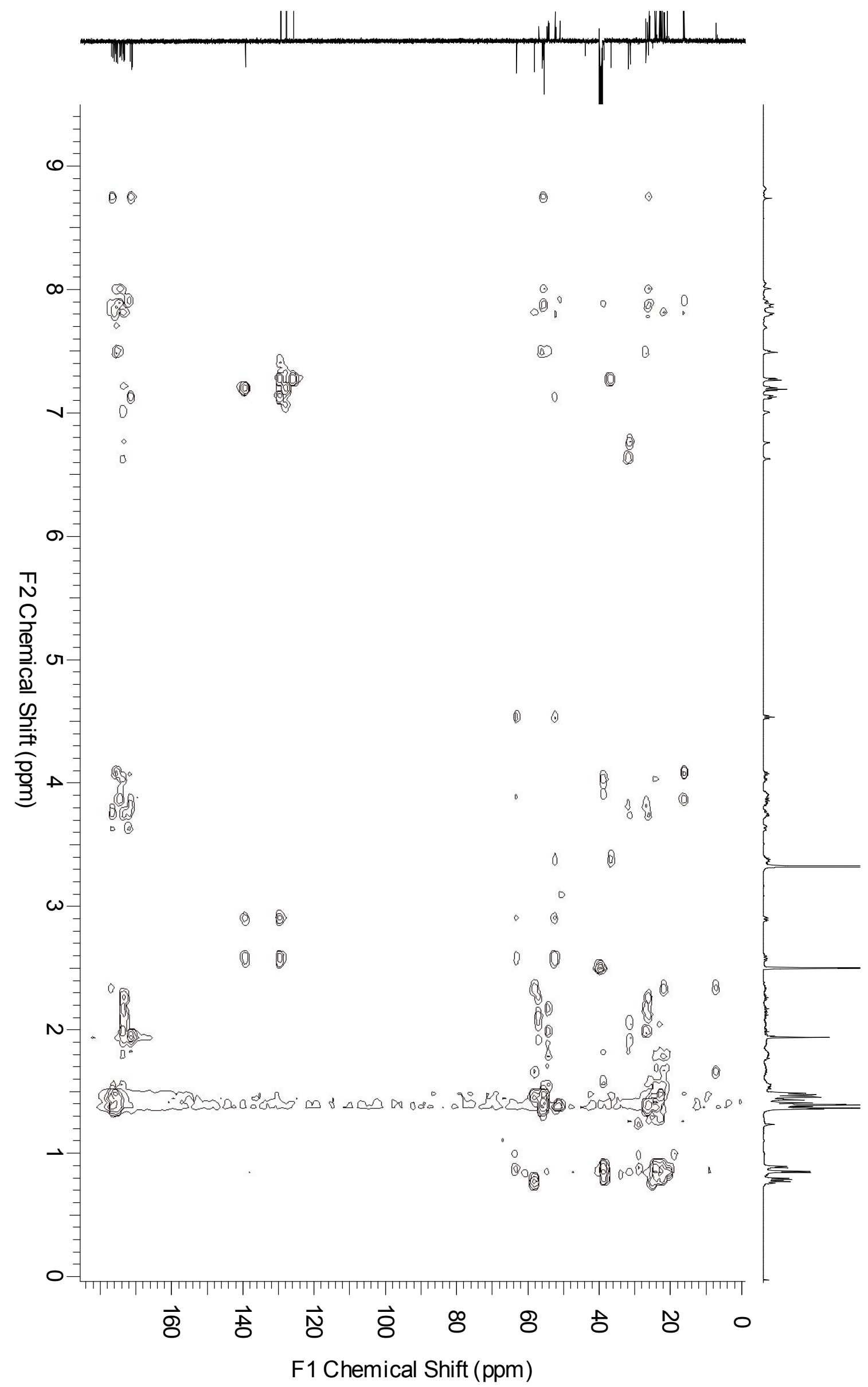


Table S5. ${ }^{1} \mathrm{H}$ and ${ }^{13} \mathrm{C}$ NMR of pentadecaibin IV (4) $\left({ }^{1} \mathrm{H} 600 \mathrm{MHz},{ }^{13} \mathrm{C} 150 \mathrm{MHz}\right.$ DMSO-d6, $298^{\circ} \mathrm{K}$ )

\begin{tabular}{|c|c|c|c|c|c|c|c|c|}
\hline $\begin{array}{l}\text { Pos. } \\
\text { Ac-Aib }^{1}\end{array}$ & $\delta_{C}$, type & $\delta_{\mathrm{H}}$, mult. $(J$ in $\mathrm{Hz})$ & $\begin{array}{l}\text { Pos. } \\
\text { Gln }^{6}\end{array}$ & $\delta_{c}$, type & $\delta_{\mathrm{H}}$, mult. $(J$ in $\mathrm{Hz})$ & $\begin{array}{l}\text { Pos. } \\
\text { Aib }^{11}\end{array}$ & $\delta_{C}$, type & $\delta_{\mathrm{H}}$, mult. $(J$ in $\mathrm{Hz})$ \\
\hline $\mathrm{C}=\mathrm{O}$ & $176.4, \mathrm{C}$ & - & $\mathrm{C}=\mathrm{O}$ & 173.2, C & - & $\mathrm{C}=\mathrm{O}$ & 175.1, C & - \\
\hline $\mathrm{N}-\mathrm{H}$ & - & $8.73, \mathrm{~s}$ & $\mathrm{~N}-\mathrm{H}$ & - & $7.78, \mathrm{~m}$ & $\mathrm{~N}-\mathrm{H}$ & - & $7.87, \mathrm{~s}$ \\
\hline$\alpha \mathrm{C}$ & $55.6, \mathrm{C}$ & - & $\alpha \mathrm{CH}$ & $56.9, \mathrm{CH}$ & $3.73, \mathrm{~m}$ & $\alpha \mathrm{C}$ & $55.5, \mathrm{C}$ & - \\
\hline$\beta \mathrm{CH}_{3}$ & $(*), \mathrm{CH}_{3}$ & $\left({ }^{\circ}\right) \mathrm{s}$ & $\beta \mathrm{CH}_{2}$ & 26.1, $\mathrm{CH}_{2}$ & $1.88, \mathrm{~m}$ & $\beta \mathrm{CH}_{3}$ & $(*), \mathrm{CH}_{3}$ & $\left({ }^{\circ}\right) \mathrm{s}$ \\
\hline$\beta^{\prime} \mathrm{CH}_{3}$ & $(*), \mathrm{CH}_{3}$ & $\left({ }^{\circ}\right) \mathrm{s}$ & & - & $1.78, \mathrm{~m}$ & $\beta^{\prime} \mathrm{CH}_{3}$ & $(*), \mathrm{CH}_{3}$ & $\left({ }^{\circ}\right) \mathrm{s}$ \\
\hline$\underline{\mathrm{COCH}}_{3}$ & 171.1, C & - & $\gamma \mathrm{CH}_{2}$ & $31.3, \mathrm{CH}_{2}$ & $2.24, \mathrm{~m}$ & & & \\
\hline \multirow[t]{4}{*}{$\mathrm{COCH}_{3}$} & $23.1, \mathrm{CH}_{3}$ & $1.93, \mathrm{~s}$ & & - & $2.11, \mathrm{~m}$ & & & \\
\hline & & & $\delta \mathrm{C}=\mathrm{O}$ & 173.1, C & - & & & \\
\hline & & & $\varepsilon \mathrm{NH}_{2}$ an & & $7.18, \mathrm{~s}$ & & & \\
\hline & & & $\varepsilon \mathrm{NH}_{2}$ sy & & $6.75, \mathrm{~s}$ & & & \\
\hline Gly $^{2}$ & & & $\mathrm{Iva}^{7}$ & & & $\mathrm{Aib}^{12}$ & & \\
\hline $\mathrm{C}=\mathrm{O}$ & $171.6, \mathrm{C}$ & - & $\mathrm{C}=\mathrm{O}$ & $176.8, \mathrm{C}$ & - & $\mathrm{C}=\mathrm{O}$ & $175.4, \mathrm{C}$ & - \\
\hline $\mathrm{N}-\mathrm{H}$ & - & 8.89, brs & $\mathrm{N}-\mathrm{H}$ & - & $7.78, \mathrm{~s}$ & $\mathrm{~N}-\mathrm{H}$ & - & $7.48, \mathrm{~s}$ \\
\hline \multirow[t]{5}{*}{$\alpha \mathrm{CH}_{2}$} & $43.9, \mathrm{CH}_{2}$ & 3.75, dd $(16.3,5.9)(\operatorname{proS})$ & $\alpha \mathrm{C}$ & $58.1, \mathrm{C}$ & - & $\alpha \mathrm{C}$ & $56.0, \mathrm{C}$ & - \\
\hline & - & 3.63, dd $(16.3,4.7)$ (pro R) & $\beta \mathrm{CH}_{2}$ & $24.9, \mathrm{CH}_{2}$ & $2.31, \mathrm{~m}$ & $\beta \mathrm{CH}_{3}$ & $(*), \mathrm{CH}_{3}$ & $\left({ }^{\circ}\right) \mathrm{s}$ \\
\hline & & & & - & $1.65, \mathrm{~m}$ & $\beta^{\prime} \mathrm{CH}_{3}$ & $(*), \mathrm{CH}_{3}$ & $\left({ }^{\circ}\right) \mathrm{s}$ \\
\hline & & & $\beta \mathrm{CH}_{3}$ & $21.8, \mathrm{CH}_{3}$ & $1.48, \mathrm{~s}$ & & & \\
\hline & & & $\gamma \mathrm{CH}_{3}$ & $7.2, \mathrm{CH}_{3}$ & 0.76 , dd $(7.6,7.6)$ & & & \\
\hline $\mathrm{Ala}^{3}$ & & & Leu $^{8}$ & & & $\mathrm{Aib}^{13}$ & & \\
\hline $\mathrm{C}=\mathrm{O}$ & $175.2, \mathrm{C}$ & - & $\mathrm{C}=\mathrm{O}$ & $174, \mathrm{C}$ & - & $\mathrm{C}=\mathrm{O}$ & $174.5, \mathrm{C}$ & - \\
\hline $\mathrm{N}-\mathrm{H}$ & - & $7.89, \mathrm{~d}(6.0)$ & $\mathrm{N}-\mathrm{H}$ & - & $7.85, \mathrm{~m}$ & $\mathrm{~N}-\mathrm{H}$ & - & $7.85, \mathrm{~s}$ \\
\hline$\alpha \mathrm{CH}$ & $51.0, \mathrm{CH}$ & $4.07, \mathrm{dq}(6.0,7.0)$ & $\alpha \mathrm{CH}$ & $54.7, \mathrm{CH}$ & $3.87, \mathrm{~m}$ & $\alpha \mathrm{C}$ & $55.8, \mathrm{C}$ & - \\
\hline \multirow[t]{5}{*}{$\beta \mathrm{CH}_{3}$} & $16.1, \mathrm{CH}_{3}$ & $1.35, \mathrm{~d}(7.0)$ & $\beta \mathrm{CH}_{2}$ & $38.7, \mathrm{CH}_{2}$ & $1.70, \mathrm{~m}$ & $\beta \mathrm{CH}_{3}$ & $(*), \mathrm{CH}_{3}$ & $\left({ }^{\circ}\right) \mathrm{s}$ \\
\hline & & & & - & $1.52, \mathrm{~m}$ & $\beta^{\prime} \mathrm{CH}_{3}$ & $(*), \mathrm{CH}_{3}$ & $\left({ }^{\circ}\right) \mathrm{s}$ \\
\hline & & & $\gamma \mathrm{CH}$ & $24.1, \mathrm{CH}$ & $1.79, \mathrm{~m}$ & & & \\
\hline & & & $\delta \mathrm{CH}_{3}$ & $21.5, \mathrm{CH}_{3}(* *)$ & $0.85, \mathrm{~d}(6.4)$ & & & \\
\hline & & & $\delta^{\prime} \mathrm{CH}_{3}$ & $20.8, \mathrm{CH}_{3}$ & $0.78, \mathrm{~d}(6.6)$ & & & \\
\hline Leu $^{4}$ & & & $\mathrm{Aib}^{9}$ & & & $\mathrm{Gln}^{14}$ & & \\
\hline $\mathrm{C}=\mathrm{O}$ & 173.6, C & - & $\mathrm{C}=\mathrm{O}$ & 175.6, C & - & $\mathrm{C}=\mathrm{O}$ & $171.2, \mathrm{C}$ & - \\
\hline $\mathrm{N}-\mathrm{H}$ & - & $7.68, \mathrm{~d}(5.9)$ & $\mathrm{N}-\mathrm{H}$ & - & $8.00, \mathrm{~s}$ & $\mathrm{~N}-\mathrm{H}$ & - & $7.49 \mathrm{dd},(6.5)$ \\
\hline$\alpha \mathrm{CH}$ & $54.0, \mathrm{CH}$ & 4.03, ddd $(7.0,7.0,5.9)$ & $\alpha \mathrm{C}$ & $55.5, \mathrm{C}$ & - & $\alpha \mathrm{CH}$ & $54.3, \mathrm{CH}$ & $3.83, \mathrm{~m}$ \\
\hline \multirow[t]{2}{*}{$\beta \mathrm{CH}_{2}$} & $38.7, \mathrm{CH}_{2}$ & $1.76, \mathrm{~m}$ & $\beta \mathrm{CH}_{3}$ & $(*), \mathrm{CH}_{3}$ & $\left({ }^{\circ}\right) \mathrm{s}$ & $\beta \mathrm{CH}_{2}$ & $26.8, \mathrm{CH}_{2}$ & $2.04, \mathrm{~m}$ \\
\hline & - & $1.65, \mathrm{~m}$ & $\beta^{\prime} \mathrm{CH}_{3}$ & $(*), \mathrm{CH}_{3}$ & $\left({ }^{\circ}\right) \mathrm{s}$ & & - & $1.92, \mathrm{~m}$ \\
\hline$\gamma \mathrm{CH}$ & $24.3, \mathrm{CH}$ & $1.70, \mathrm{~m}$ & & & & $\gamma \mathrm{CH}_{2}$ & $31.8, \mathrm{CH}_{2}$ & $2.14, \mathrm{~m}$ \\
\hline$\delta \mathrm{CH}_{3}$ & $22.3, \mathrm{CH}_{3}(* *)$ & $0.84, \mathrm{~d}(6.4)$ & & & & & - & $1.97, \mathrm{~m}$ \\
\hline \multirow[t]{3}{*}{$\delta^{\prime} \mathrm{CH}_{3}$} & $21.8, \mathrm{CH}_{3}$ & $0.88, \mathrm{~d}(6.4)$ & & & & $\delta \mathrm{C}=\mathrm{O}$ & 173.4 & \\
\hline & & & & & & $\varepsilon \mathrm{NH}_{2 \text { anti }}$ & - & $6.99, \mathrm{~s}$ \\
\hline & & & & & & $\varepsilon \mathrm{NH}_{2}$ syn & - & $6.62, \mathrm{~s}$ \\
\hline $\mathrm{Aib}^{5}$ & & & $\mathrm{Ala}^{10}$ & & & Pheol $^{15}$ & & \\
\hline $\mathrm{C}=\mathrm{O}$ & 176.0, C & - & $\mathrm{C}=\mathrm{O}$ & $174.3, \mathrm{C}$ & - & $\mathrm{NH}$ & - & $7.12, \mathrm{~m}$ \\
\hline $\mathrm{N}-\mathrm{H}$ & - & $8.04, \mathrm{~s}$ & $\mathrm{~N}-\mathrm{H}$ & - & $7.81, \mathrm{~m}$ & $\alpha \mathrm{CH}$ & $52.4, \mathrm{CH}$ & $3.86, \mathrm{~m}$ \\
\hline$\alpha \mathrm{C}$ & $55.5, \mathrm{C}$ & - & $\alpha \mathrm{CH}$ & $52.0, \mathrm{CH}$ & $3.83, \mathrm{~m}$ & $\beta \mathrm{CH}_{2}$ & $36.7, \mathrm{CH}_{2}$ & $2.89, \mathrm{dd}(13.6,4.5)$ \\
\hline$\beta \mathrm{CH}_{3}$ & $(*), \mathrm{CH}_{3}$ & $\left({ }^{\circ}\right) \mathrm{s}$ & $\beta \mathrm{CH}_{3}$ & $16.3, \mathrm{CH}_{3}$ & $1.35, \mathrm{~m}$ & & - & $2.57, \mathrm{dd}(13.6,9.2)$ \\
\hline \multirow[t]{7}{*}{$\beta^{\prime} \mathrm{CH}_{3}$} & $(*), \mathrm{CH}_{3}$ & $\left({ }^{\circ}\right) \mathrm{s}$ & & & & $\beta \mathrm{CH}_{2} \mathrm{OH}$ & $63.2, \mathrm{CH}_{2}$ & $3.37, \mathrm{~m}$ \\
\hline & & & & & & & - & $3.37, \mathrm{~m}$ \\
\hline & & & & & & $\mathrm{OH}$ & - & $4.52, \mathrm{dd}(\mathrm{t} 6.1)$ \\
\hline & & & & & & $\mathrm{C}-1$ & $139.2, \mathrm{C}$ & - \\
\hline & & & & & & $\mathrm{C}-2,6$ & $129.3, \mathrm{CH}$ & $7.26, \mathrm{~m}(\mathrm{~d})(8.3)$ \\
\hline & & & & & & $\mathrm{C}-3,5$ & $127.8, \mathrm{CH}$ & $7.13, \mathrm{~m}(\mathrm{t})(8.3)$ \\
\hline & & & & & & $\mathrm{C}-4$ & $125.7, \mathrm{CH}$ & $7.12, \mathrm{~m}(\mathrm{t})(8.3)$ \\
\hline \multicolumn{9}{|c|}{$\beta / \beta^{\prime} \mathrm{CH}_{3} \mathrm{Aib}^{1}, \mathrm{Aib}^{5}, \mathrm{Aib}^{9}, \mathrm{Aib}^{11}, \mathrm{Aib}^{12}, \mathrm{Aib}^{13}$. } \\
\hline \multicolumn{9}{|c|}{${ }^{13} \mathrm{C}(*) 26.9,26.4,26.0,26.0,25.8,25.7,2$} \\
\hline \multicolumn{9}{|c|}{$\begin{array}{r}{ }^{1} \mathrm{H}\left({ }^{\circ}\right) \\
(* *) \text { may b }\end{array}$} \\
\hline
\end{tabular}

Fig. S45. NMR key correlations of pentadecaibin IV (4) (COSY/TOCSY, HMBC and NOESY).

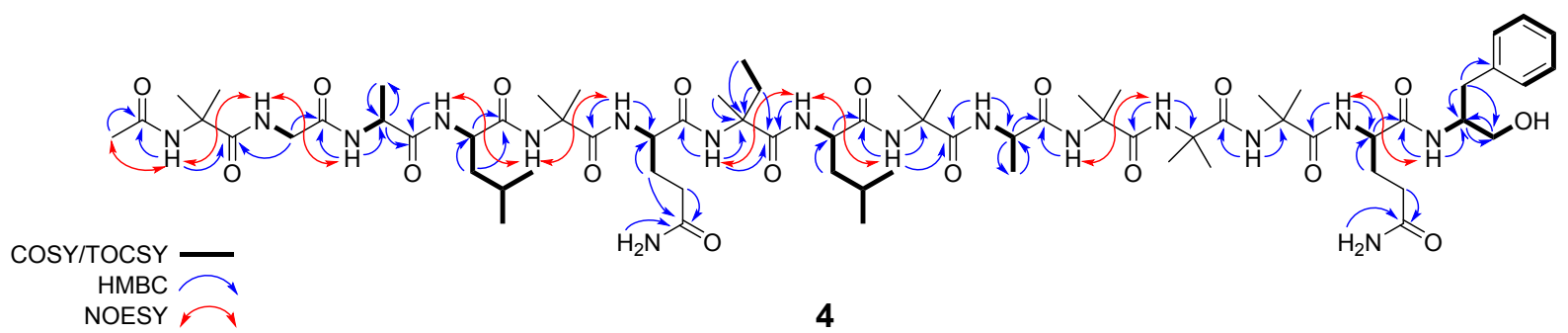


Fig. S46. CD spectrum of pentadecaibin IV (4) in $\mathrm{MeOH}$

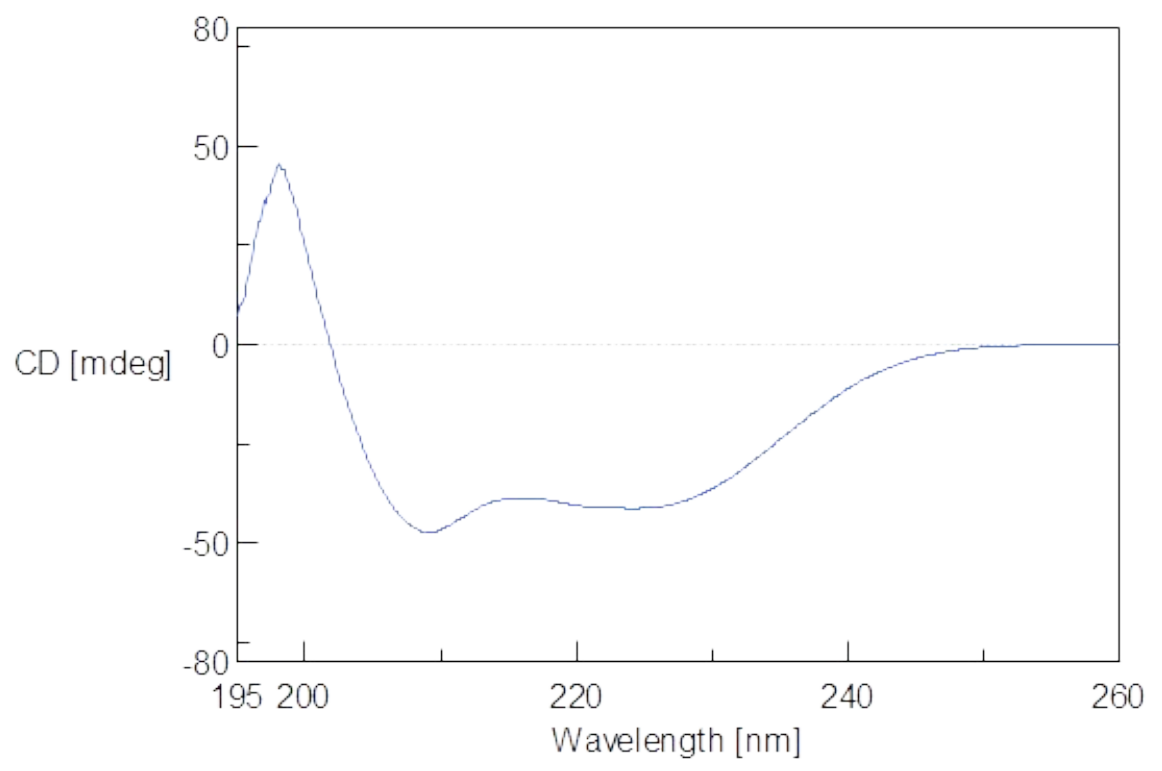


Fig. S47. ${ }^{1} \mathrm{H}$ NMR spectrum of pentadecaibin V (5) (600 MHz, DMSO-d6, $\left.298^{\circ} \mathrm{K}\right)$

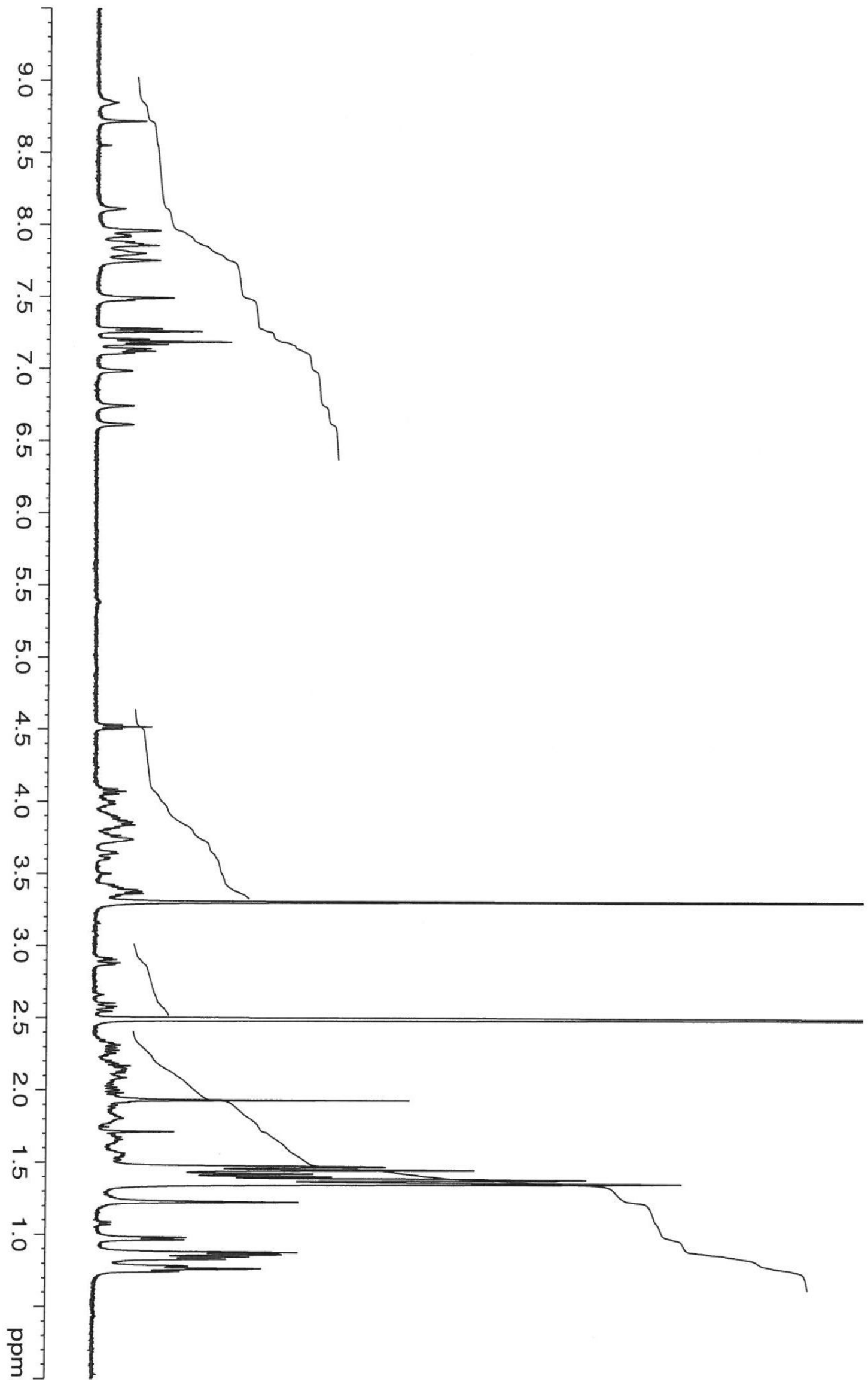


Fig. S48. ${ }^{1} \mathrm{H},{ }^{1} \mathrm{H}$ COSY spectrum of pentadecaibin V (5) $\left(600 \mathrm{MHz}, \mathrm{DMSO}-d 6,298{ }^{\circ} \mathrm{K}\right)$

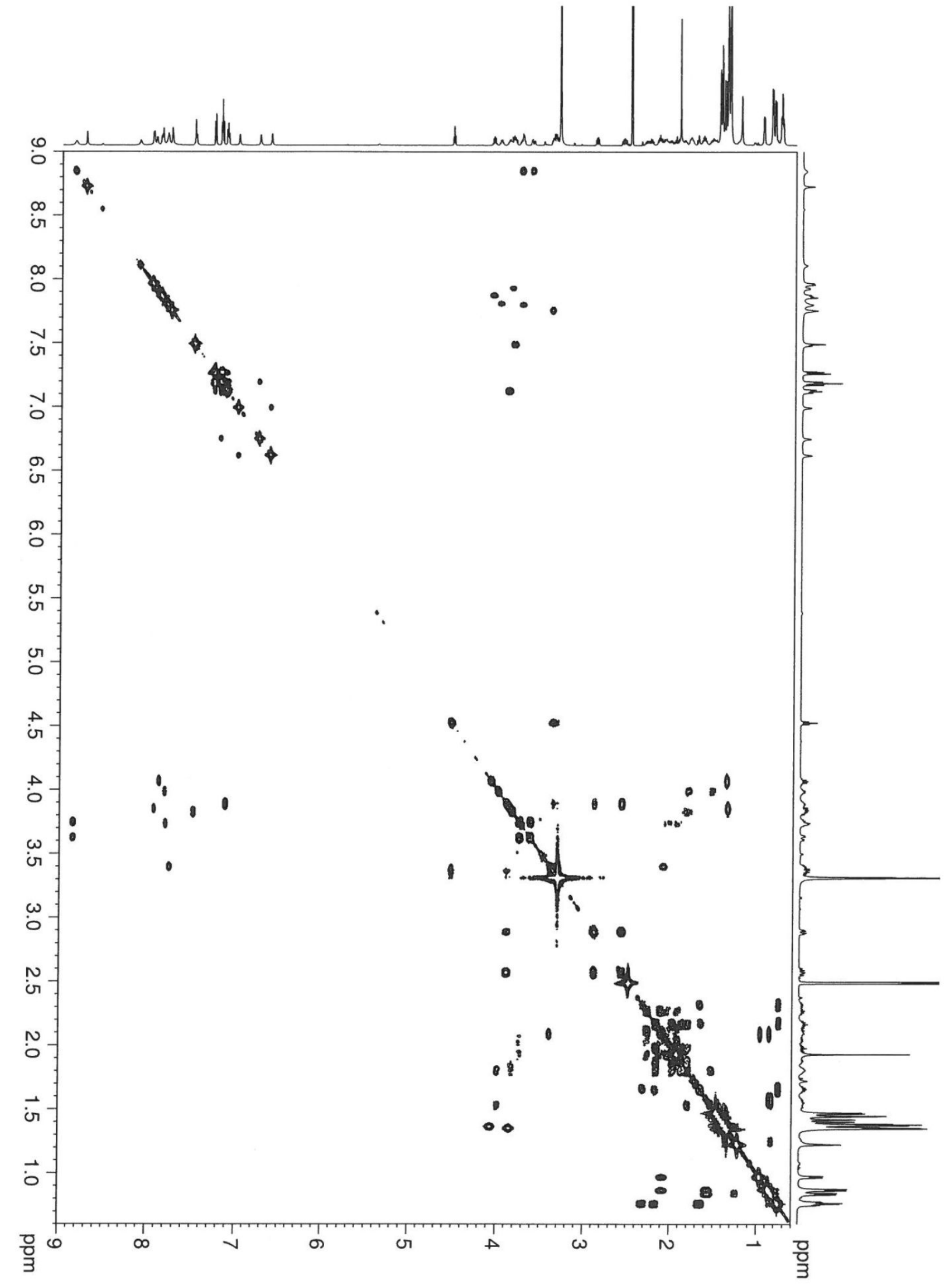


Fig. S49. ${ }^{1} \mathrm{H},{ }^{1} \mathrm{H}$ TOCSY spectrum of pentadecaibin V (5) $\left(600 \mathrm{MHz}, \mathrm{DMSO}-d 6,298{ }^{\circ} \mathrm{K}\right)$

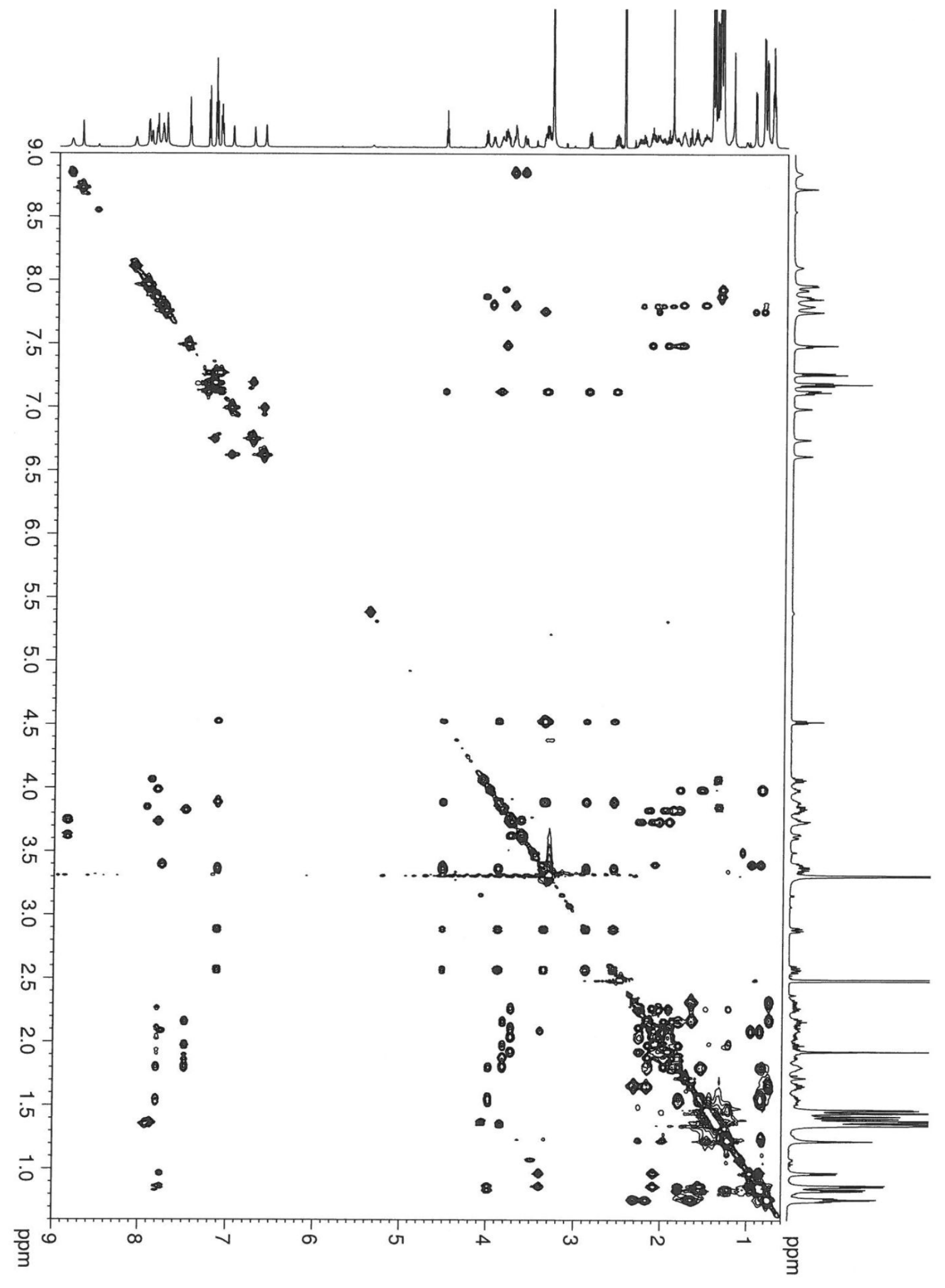


Fig. S50. ${ }^{1} \mathrm{H},{ }^{1} \mathrm{H}$ NOESY spectrum of pentadecaibin V (5) $\left(600 \mathrm{MHz}, \mathrm{DMSO}-d 6,298{ }^{\circ} \mathrm{K}\right)$

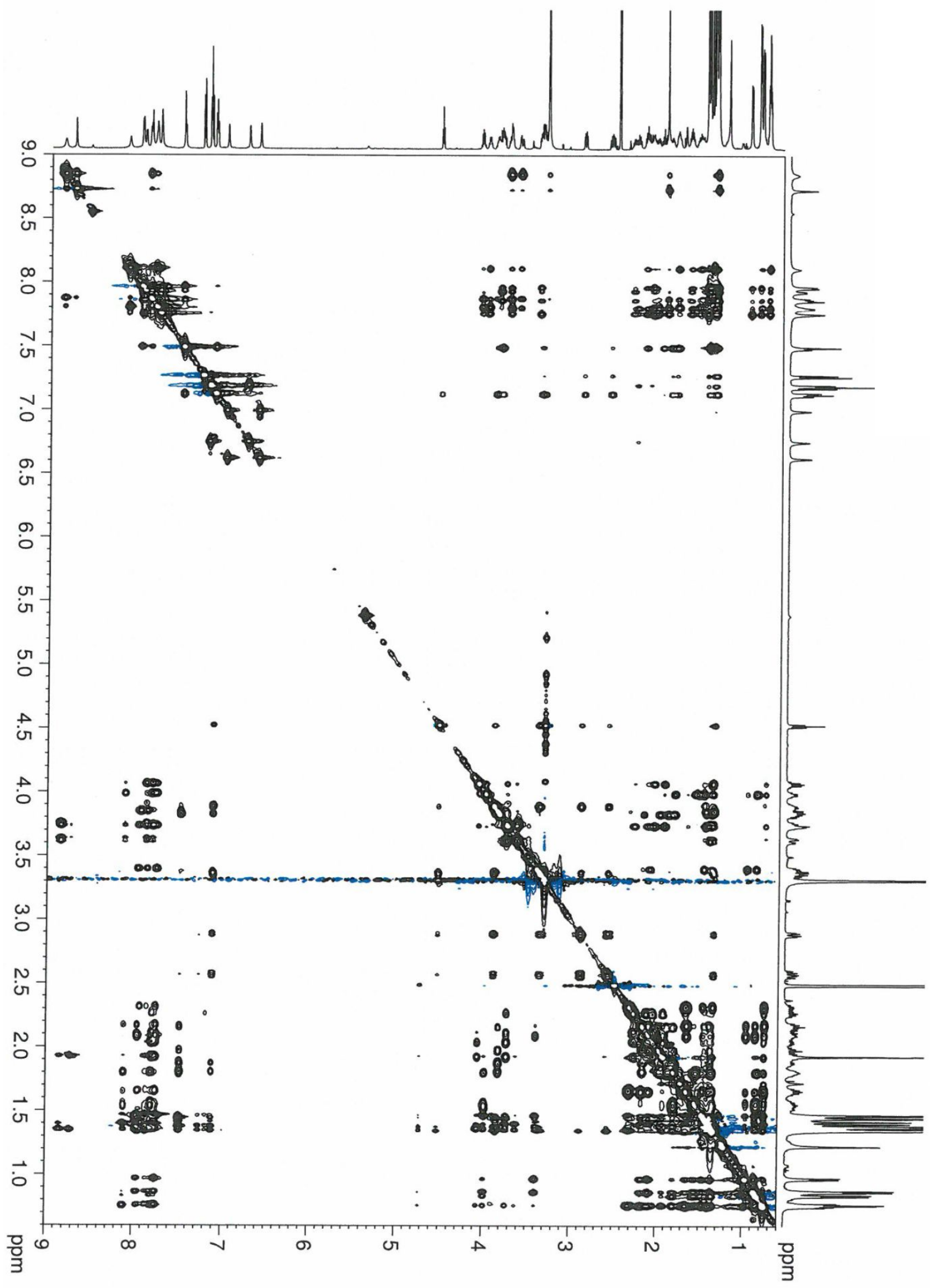


Fig. S51. DEPT-135 spectrum of pentadecaibin V (5) (150 MHz, DMSO-d6, $\left.298^{\circ} \mathrm{K}\right)$

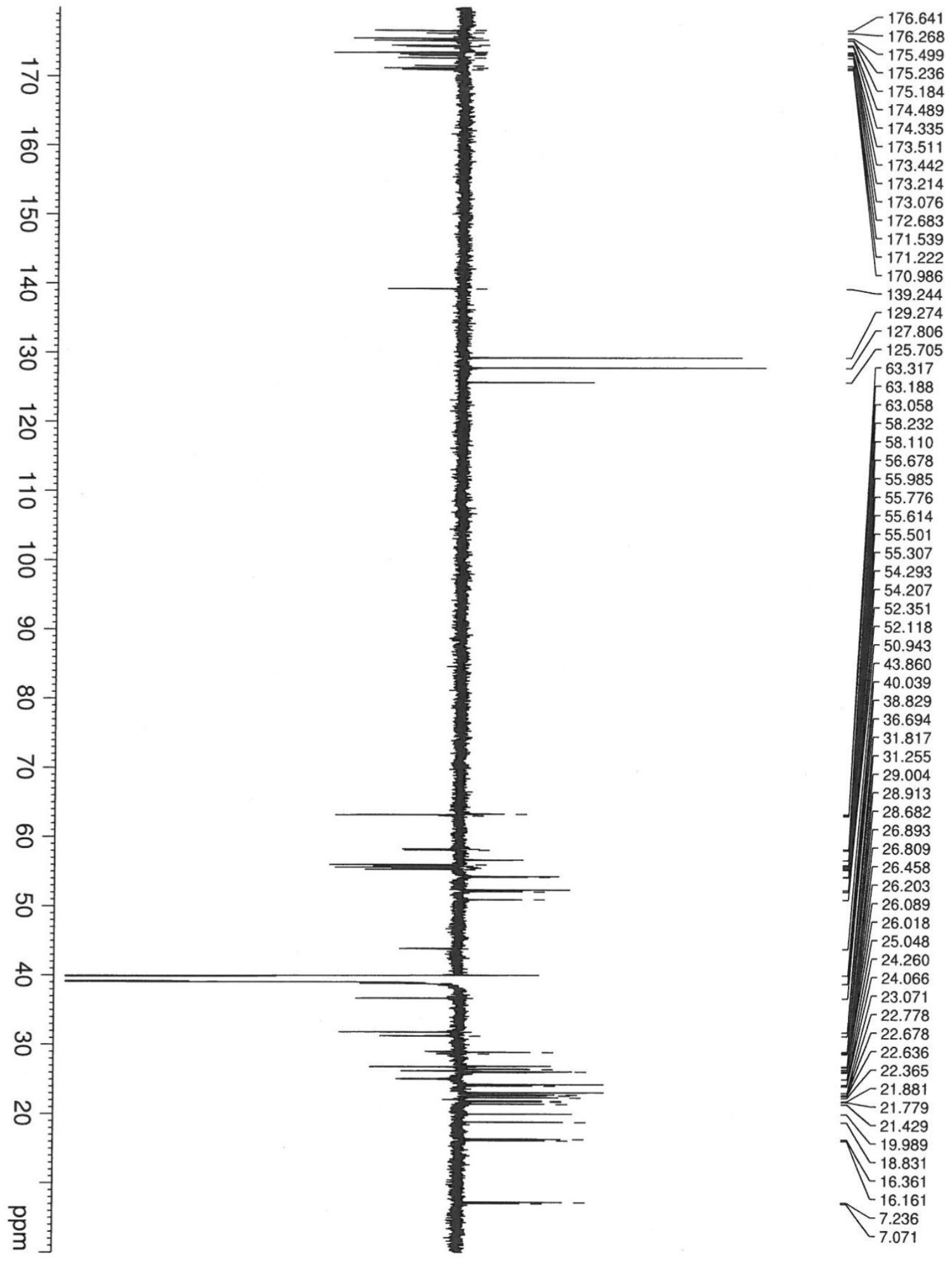


Fig. S52. ${ }^{1} \mathrm{H},{ }^{13} \mathrm{C}$ HSQC spectrum of pentadecaibin V (5) $\left(600 \mathrm{MHz}, \mathrm{DMSO}-d 6,298{ }^{\circ} \mathrm{K}\right)$

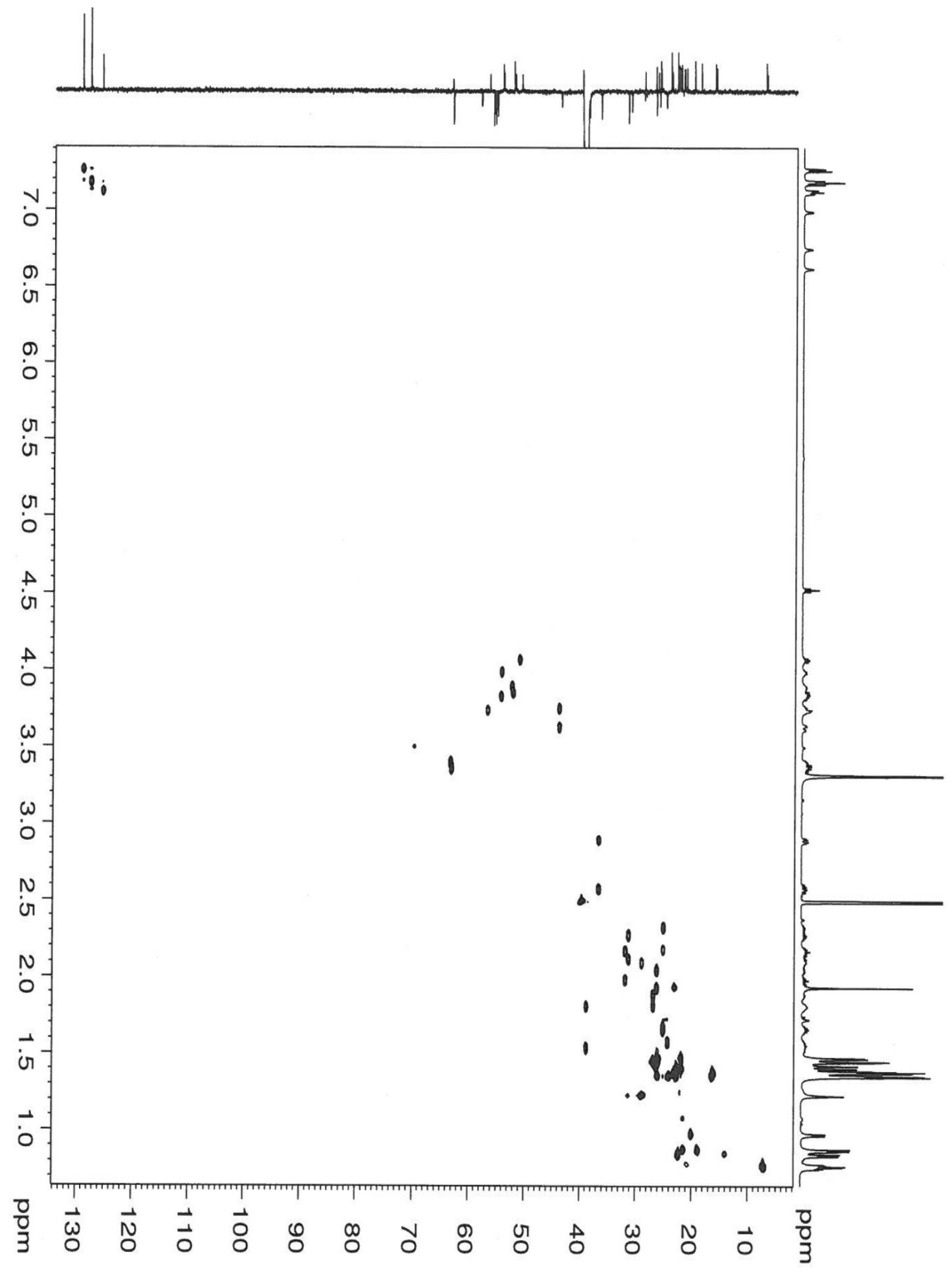


Fig. S53. ${ }^{1} \mathrm{H},{ }^{13} \mathrm{C}$ HMBC spectrum of pentadecaibin V (5) $\left(600 \mathrm{MHz}, \mathrm{DMSO}-d 6,298{ }^{\circ} \mathrm{K}\right)$

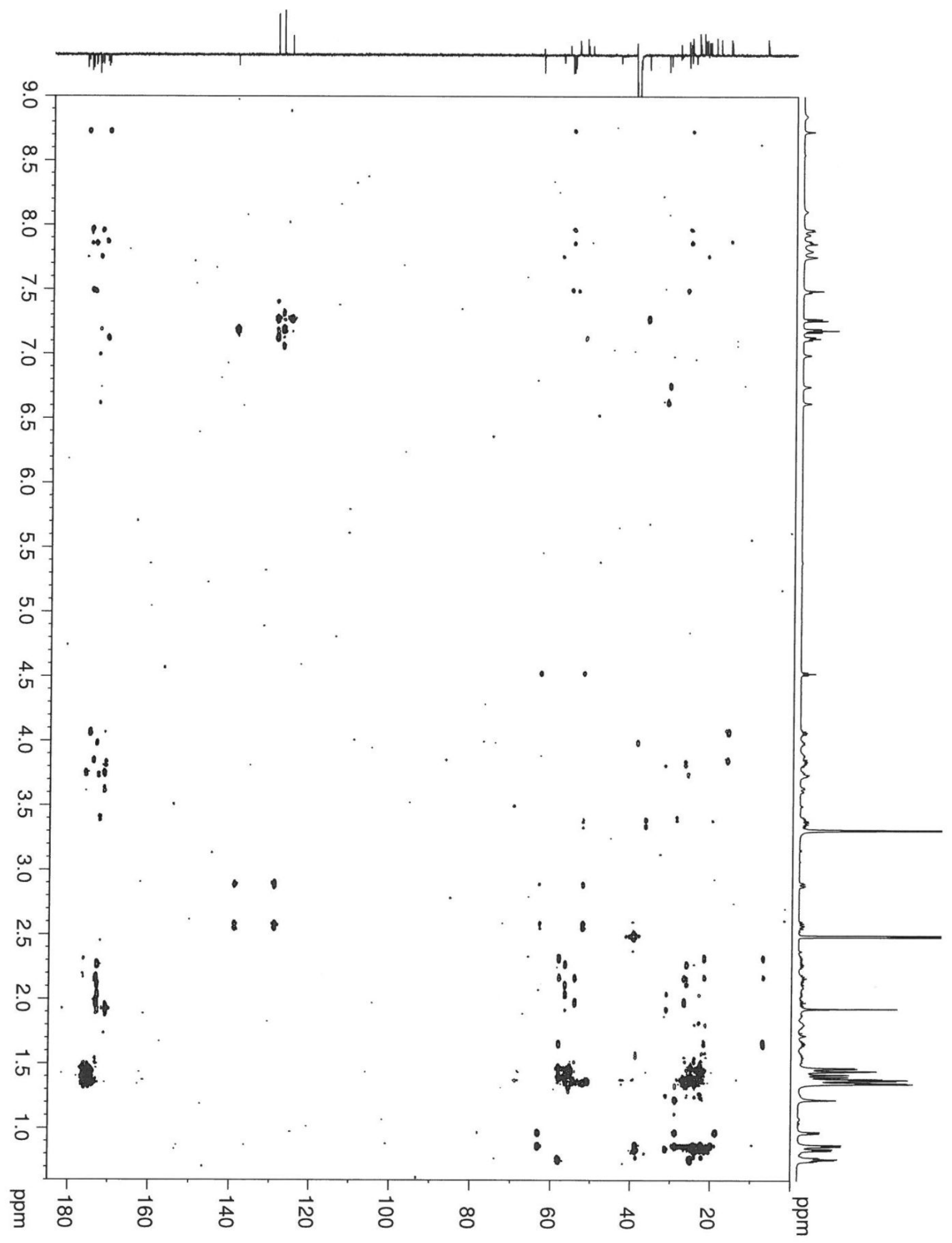


Table S6. ${ }^{1} \mathrm{H}$ and ${ }^{13} \mathrm{C}$ NMR of pentadecaibin V (5) $\left({ }^{1} \mathrm{H} 600 \mathrm{MHz},{ }^{13} \mathrm{C} 150 \mathrm{MHz}\right.$ DMSO-d6, $298^{\circ} \mathrm{K}$ )

\begin{tabular}{|c|c|c|c|c|c|c|c|c|}
\hline $\begin{array}{l}\text { Pos. } \\
\text { Ac-Aib }\end{array}$ & $\delta_{c}$, type & $\delta_{\mathrm{H}}$, mult. $(J$ in Hz) & $\begin{array}{l}\text { Pos. } \\
\text { Gln }^{6}\end{array}$ & $\delta_{c}$, type & $\delta_{\mathrm{H}}$, mult. $(J$ in Hz) & $\begin{array}{l}\text { Pos. } \\
\text { Aib }^{11}\end{array}$ & $\delta_{c}$, type & $\delta_{\mathrm{H}}$, mult. $(J$ in Hz) \\
\hline $\mathrm{C}=\mathrm{O}$ & $176.3, \mathrm{C}$ & - & $\mathrm{C}=\mathrm{O}$ & $173.1, \mathrm{C}$ & - & $\mathrm{C}=\mathrm{O}$ & $175.2, \mathrm{C}$ & - \\
\hline $\mathrm{N}-\mathrm{H}$ & - & $8.72, \mathrm{~s}$ & $\mathrm{~N}-\mathrm{H}$ & - & $7.80, \mathrm{~m}$ & $\mathrm{~N}-\mathrm{H}$ & - & $7.85, \mathrm{~s}$ \\
\hline$\alpha \mathrm{C}$ & $55.6, \mathrm{C}$ & - & $\alpha \mathrm{CH}$ & $56.7, \mathrm{CH}$ & $3.74, \mathrm{~m}$ & $\alpha \mathrm{C}$ & $55.5, \mathrm{C}(* *)$ & - \\
\hline$\beta \mathrm{CH}_{3}$ & $(*), \mathrm{CH}_{3}$ & $\left({ }^{\circ}\right) \mathrm{s}$ & $\beta \mathrm{CH}_{2}$ & $26.4, \mathrm{CH}_{2}$ & $2.04, \mathrm{~m}$ & $\beta \mathrm{CH}_{3}$ & $(*), \mathrm{CH}_{3}$ & $\left({ }^{\circ}\right) \mathrm{s}$ \\
\hline$\beta^{\prime} \mathrm{CH}_{3}$ & $(*), \mathrm{CH}_{3}$ & $\left({ }^{\circ}\right) \mathrm{s}$ & & - & $1.93, \mathrm{~m}$ & $\beta^{\prime} \mathrm{CH}_{3}$ & $(*), \mathrm{CH}_{3}$ & $\left({ }^{\circ}\right) \mathrm{s}$ \\
\hline $\mathrm{COCH}_{3}$ & $171.0, \mathrm{C}$ & - & $\gamma \mathrm{CH}_{2}$ & $31.3, \mathrm{CH}_{2}$ & $2.27, \mathrm{~m}$ & & & \\
\hline \multirow[t]{4}{*}{$\mathrm{COCH}_{3}$} & $23.1, \mathrm{CH}_{3}$ & $1.93, \mathrm{~s}$ & & - & $2.10, \mathrm{~m}$ & & & \\
\hline & & & $\delta \mathrm{C}=\mathrm{O}$ & $173.2, \mathrm{C}$ & - & & & \\
\hline & & & $\varepsilon \mathrm{NH}_{2}$ anti & - & $7.18, \mathrm{~s}$ & & & \\
\hline & & & $\varepsilon \mathrm{NH}_{2}$ syn & - & $6.74, \mathrm{~s}$ & & & \\
\hline Gly $^{2}$ & & & $\operatorname{Iva}^{7}$ & & & $\mathrm{Aib}^{12}$ & & \\
\hline $\mathrm{C}=\mathrm{O}$ & $171.5, \mathrm{C}$ & - & $\mathrm{C}=\mathrm{O}$ & 176.6, C & - & $\mathrm{C}=\mathrm{O}$ & $175.5, \mathrm{C}$ & - \\
\hline $\mathrm{N}-\mathrm{H}$ & - & 8.84, brs & $\mathrm{N}-\mathrm{H}$ & - & $7.75, \mathrm{~s}$ & $\mathrm{~N}-\mathrm{H}$ & - & $7.49, \mathrm{~s}$ \\
\hline \multirow[t]{5}{*}{$\alpha \mathrm{CH}_{2}$} & $43.9, \mathrm{CH}_{2}$ & $3.75, \mathrm{~m}($ pro S) & $\alpha \mathrm{C}$ & $58.2, \mathrm{C}$ & - & $\alpha \mathrm{C}$ & $56, \mathrm{C}$ & - \\
\hline & - & $3.62, \mathrm{dd}(16.1,5.0)$ (pro R) & $\beta \mathrm{CH}_{2}$ & $25.0, \mathrm{CH}_{2}$ & $2.32, \mathrm{~m}$ & $\beta \mathrm{CH}_{3}$ & $(*), \mathrm{CH}_{3}$ & $\left({ }^{\circ}\right) \mathrm{s}$ \\
\hline & & & & - & $1.65, \mathrm{~m}$ & $\beta^{\prime} \mathrm{CH}_{3}$ & $(*), \mathrm{CH}_{3}$ & $\left({ }^{\circ}\right) \mathrm{s}$ \\
\hline & & & $\beta \mathrm{CH}_{3}$ & $21.9, \mathrm{CH}_{3}$ & $1.48, \mathrm{~s}$ & & & \\
\hline & & & $\gamma \mathrm{CH}_{3}$ & $7.1, \mathrm{CH}_{3}$ & $0.75, \mathrm{dd}(7.5,7.5)$ & & & \\
\hline $\mathrm{Ala}^{3}$ & & & $\mathrm{Val}^{8}$ & & & $\mathrm{Aib}^{13}$ & & \\
\hline $\mathrm{C}=\mathrm{O}$ & $175.3, \mathrm{C}$ & - & $\mathrm{C}=\mathrm{O}$ & $172.7, \mathrm{C}$ & - & $\mathrm{C}=\mathrm{O}$ & $174.5, \mathrm{C}$ & - \\
\hline $\mathrm{N}-\mathrm{H}$ & - & $7.87, \mathrm{~d}(6.0)$ & $\mathrm{N}-\mathrm{H}$ & - & $7.75, \mathrm{~m}$ & $\mathrm{~N}-\mathrm{H}$ & - & $7.96, \mathrm{~s}$ \\
\hline$\alpha \mathrm{CH}$ & $50.9, \mathrm{CH}$ & $4.07, \mathrm{dq}(6.0,7.0)$ & $\alpha \mathrm{CH}$ & $63.3, \mathrm{CH}$ & $3.39, \mathrm{~m}$ & $\alpha \mathrm{C}$ & $55.8, \mathrm{C}(* *)$ & - \\
\hline \multirow[t]{3}{*}{$\beta \mathrm{CH}_{3}$} & $16.2, \mathrm{CH}_{3}$ & $1.37, \mathrm{~d}(7.0)$ & $\beta \mathrm{CH}$ & $28.9, \mathrm{CH}$ & $2.10, \mathrm{~m}$ & $\beta \mathrm{CH}_{3}$ & $(*), \mathrm{CH}_{3}$ & $\left({ }^{\circ}\right) \mathrm{s}$ \\
\hline & & & $\gamma \mathrm{CH}_{3}$ & $18.8, \mathrm{CH}_{3}$ & $0.97, \mathrm{~d}(6.3)$ & $\beta^{\prime} \mathrm{CH}_{3}$ & $(*), \mathrm{CH}_{3}$ & $\left({ }^{\circ}\right) \mathrm{s}$ \\
\hline & & & $\gamma^{\prime} \mathrm{CH}_{3}$ & $20.0, \mathrm{CH}_{3}$ & $0.86, \mathrm{~d}(6.5)$ & & & \\
\hline $\mathrm{Leu}^{4}$ & & & $\mathrm{Aib}^{9}$ & & & $\mathrm{Gln}^{14}$ & & \\
\hline $\mathrm{C}=\mathrm{O}$ & 173.5, C & - & $\mathrm{C}=\mathrm{O}$ & $175.5, \mathrm{C}$ & - & $\mathrm{C}=\mathrm{O}$ & $171.2, \mathrm{C}$ & - \\
\hline $\mathrm{N}-\mathrm{H}$ & - & $7.80, \mathrm{~d}(8.0)$ & $\mathrm{N}-\mathrm{H}$ & - & $7.95, \mathrm{~s}$ & $\mathrm{~N}-\mathrm{H}$ & - & $7.48, \mathrm{~d}(6.5)$ \\
\hline$\alpha \mathrm{CH}$ & $54.2, \mathrm{CH}$ & $3.98, \operatorname{ddd}(8.0,8.0,8.0)$ & $\alpha \mathrm{C}$ & $55.3, \mathrm{C}(* *)$ & - & $\alpha \mathrm{CH}$ & $54.3, \mathrm{CH}$ & $3.84, \mathrm{~m}$ \\
\hline \multirow[t]{2}{*}{$\beta \mathrm{CH}_{2}$} & $38.8, \mathrm{CH}_{2}$ & $1.80, \mathrm{~m}$ & $\beta \mathrm{CH}_{3}$ & $(*), \mathrm{CH}_{3}$ & $\left({ }^{\circ}\right) \mathrm{s}$ & $\beta \mathrm{CH}_{2}$ & $26.8, \mathrm{CH}_{2}$ & $1.88, \mathrm{~m}$ \\
\hline & - & $1.55, \mathrm{~m}$ & $\beta^{\prime} \mathrm{CH}_{3}$ & $(*), \mathrm{CH}_{3}$ & $\left({ }^{\circ}\right) \mathrm{s}$ & & - & $1.81, \mathrm{~m}$ \\
\hline$\gamma \mathrm{CH}$ & $24.3, \mathrm{CH}$ & $1.55, \mathrm{~m}$ & & & & $\gamma \mathrm{CH}_{2}$ & $31.8, \mathrm{CH}_{2}$ & $2.14, \mathrm{~m}$ \\
\hline$\delta \mathrm{CH}_{3}$ & $22.6, \mathrm{CH}_{3}$ & $0.83, \mathrm{~d}(6.4)$ & & & & & - & $1.98, \mathrm{~m}$ \\
\hline \multirow[t]{3}{*}{$\delta^{\prime} \mathrm{CH}_{3}$} & $21.4, \mathrm{CH}_{3}$ & $0.87, \mathrm{~d}(6.4)$ & & & & $\delta \mathrm{C}=\mathrm{O}$ & $173.4, \mathrm{C}$ & \\
\hline & & & & & & $\varepsilon \mathrm{NH}_{2 \text { anti }}$ & - & $6.99, \mathrm{~s}$ \\
\hline & & & & & & $\varepsilon \mathrm{NH}_{2}$ syn & - & $6.62, \mathrm{~s}$ \\
\hline $\mathrm{Iva}^{5}$ & & & $\mathrm{Ala}^{10}$ & & & Pheol $^{15}$ & & \\
\hline $\mathrm{C}=\mathrm{O}$ & 176.6, C & - & $\mathrm{C}=\mathrm{O}$ & 174.3, C & - & $\mathrm{NH}$ & - & $7.12, \mathrm{~m}$ \\
\hline $\mathrm{N}-\mathrm{H}$ & - & $8.10, \mathrm{~s}$ & $\mathrm{~N}-\mathrm{H}$ & - & $7.92, \mathrm{~m}$ & $\alpha \mathrm{CH}$ & $52.4, \mathrm{CH}$ & $3.88, \mathrm{~m}$ \\
\hline$\alpha \mathrm{C}$ & $58.1, \mathrm{C}$ & - & $\alpha \mathrm{CH}$ & $52.1, \mathrm{CH}$ & $3.85, \mathrm{~m}$ & $\beta \mathrm{CH}_{2}$ & $36.7, \mathrm{CH}_{2}$ & 2.89, dd $(13.5,4.4)$ \\
\hline \multirow[t]{2}{*}{$\beta \mathrm{CH}_{2}$} & $25, \mathrm{CH}_{2}$ & $2.17, \mathrm{~m}$ & $\beta \mathrm{CH}_{3}$ & $16.4, \mathrm{CH}_{3}$ & $1.35, \mathrm{~d}(7.0)$ & & - & $2.57, \mathrm{dd}(13.5,9.2)$ \\
\hline & - & $1.66, \mathrm{~m}$ & & & & $\beta \mathrm{CH}_{2} \mathrm{OH}$ & $63.2, \mathrm{CH}_{2}$ & $3.37, \mathrm{~m}$ \\
\hline$\beta \mathrm{CH}_{3}$ & $21.8, \mathrm{CH}_{3}$ & $1.40, \mathrm{~s}$ & & & & & - & $3.37, \mathrm{~m}$ \\
\hline \multirow[t]{5}{*}{$\gamma \mathrm{CH}_{3}$} & $7.2, \mathrm{CH}_{3}$ & $0.76, \mathrm{dd}(7.5,7.5)$ & & & & $\mathrm{OH}$ & - & $4.52, \mathrm{dd}(6.2,6.2)$ \\
\hline & & & & & & $\mathrm{C}-1$ & $139.2, \mathrm{C}$ & - \\
\hline & & & & & & $\mathrm{C}-2,6$ & $129.3, \mathrm{CH}$ & $7.27, \mathrm{~m}(\mathrm{~d})(8.4)$ \\
\hline & & & & & & $\mathrm{C}-3,5$ & $127.8, \mathrm{CH}$ & $7.18, \mathrm{~m}(\mathrm{dd})(8.4,8.4)$ \\
\hline & & & & & & $\mathrm{C}-4$ & $125.7, \mathrm{CH}$ & $7.17, \mathrm{~m}(\mathrm{dd})(8.4,8.4)$ \\
\hline \multicolumn{9}{|c|}{$\beta / \beta^{\prime} \mathrm{CH}_{3} \mathrm{Aib}^{1}, \mathrm{Aib}^{9}, \mathrm{Aib}^{11}, \mathrm{Aib}^{12}, \mathrm{Aib}^{13}$. } \\
\hline \multicolumn{9}{|c|}{${ }^{13} \mathrm{C}(*) 26$} \\
\hline \multicolumn{9}{|c|}{$\begin{array}{l}{ }^{1} \mathrm{H}\left({ }^{\circ}\right) \\
\left.{ }^{6}\right) \\
\text { may be }\end{array}$} \\
\hline
\end{tabular}

Fig. S54. NMR key correlations of pentadecaibin V (5) (COSY/TOCSY, HMBC and NOESY).

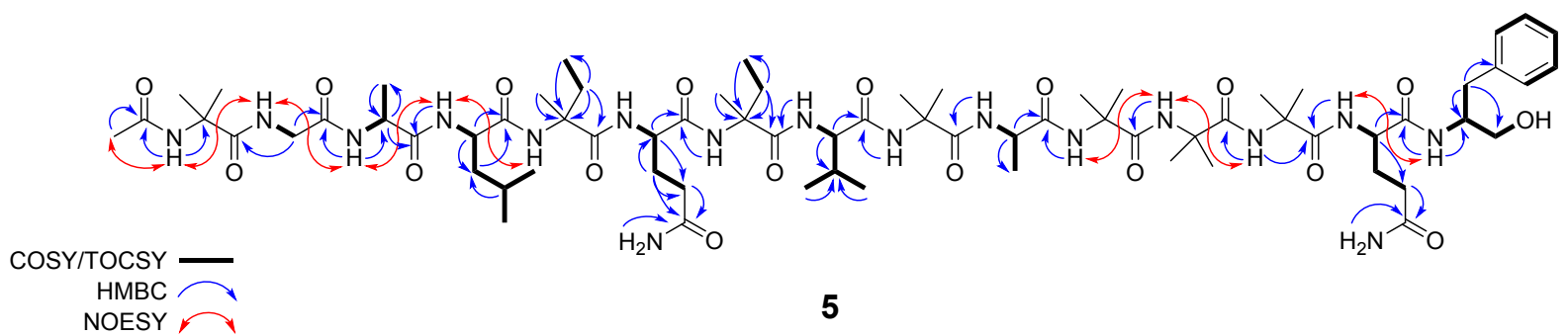


Fig. S55. CD spectrum of pentadecaibin V (5) in $\mathrm{MeOH}$

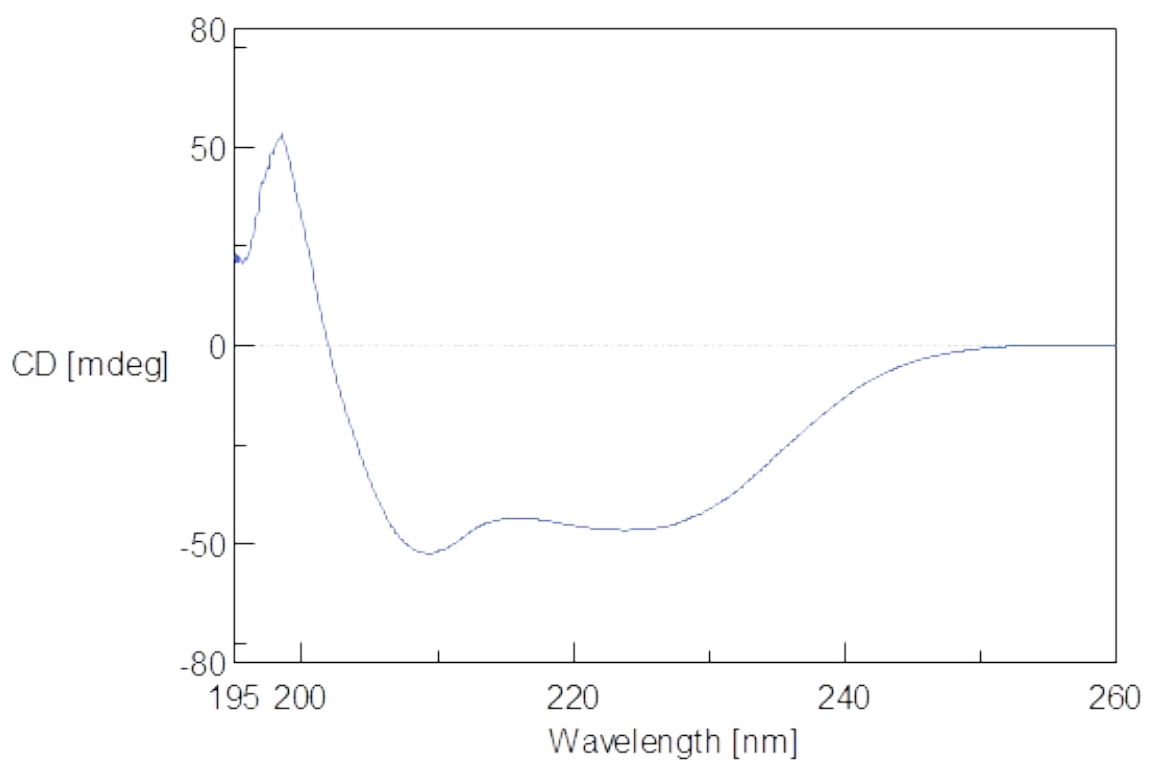


Fig. S56. Marfey's analysis of hydrolyzed pentadecaibin I (1) derivatized with L-FDAA (a) and comparison with L-FDAA derivatives of standard amino acids (b-h).

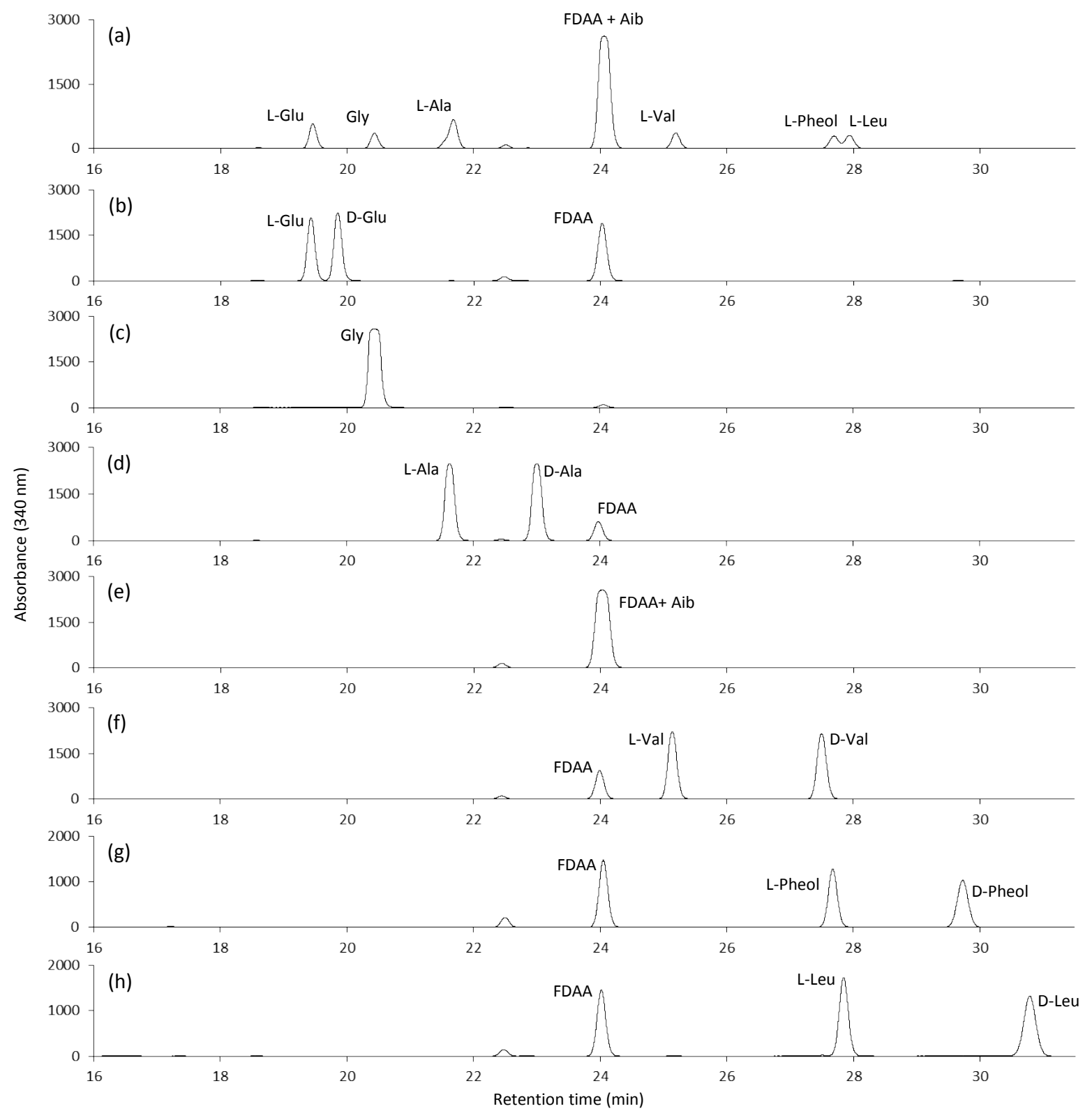


Fig. S57. Marfey's analysis of hydrolyzed pentadecaibin II (2) derivatized with L-FDAA (a) and comparison with L-FDAA derivatives of standard amino acids (b-g).

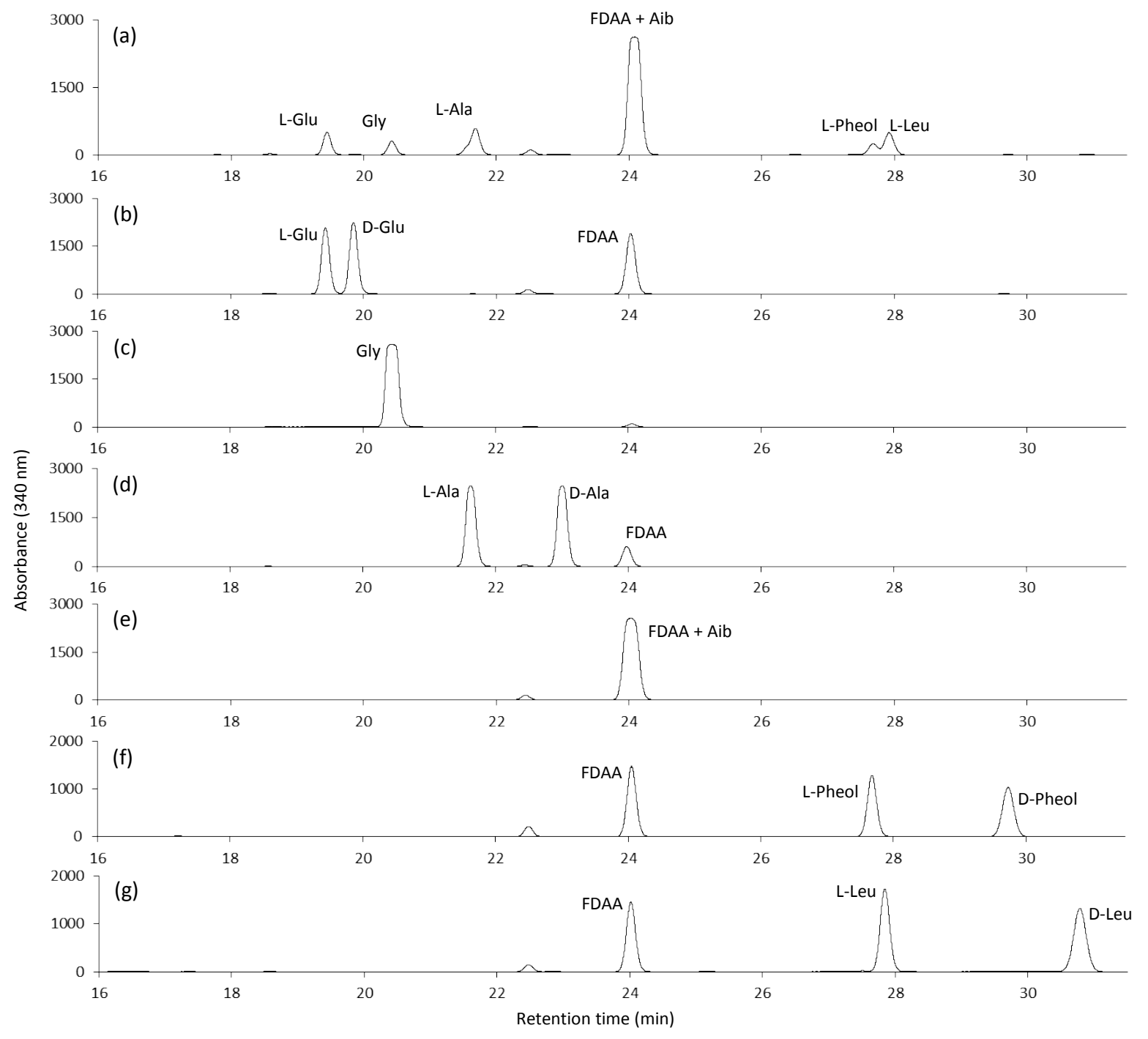


Fig. S58. Marfey's analysis of hydrolyzed pentadecaibin III (3) derivatized with L-FDAA (a) and comparison with L-FDAA derivatives of standard amino acids (b-i).

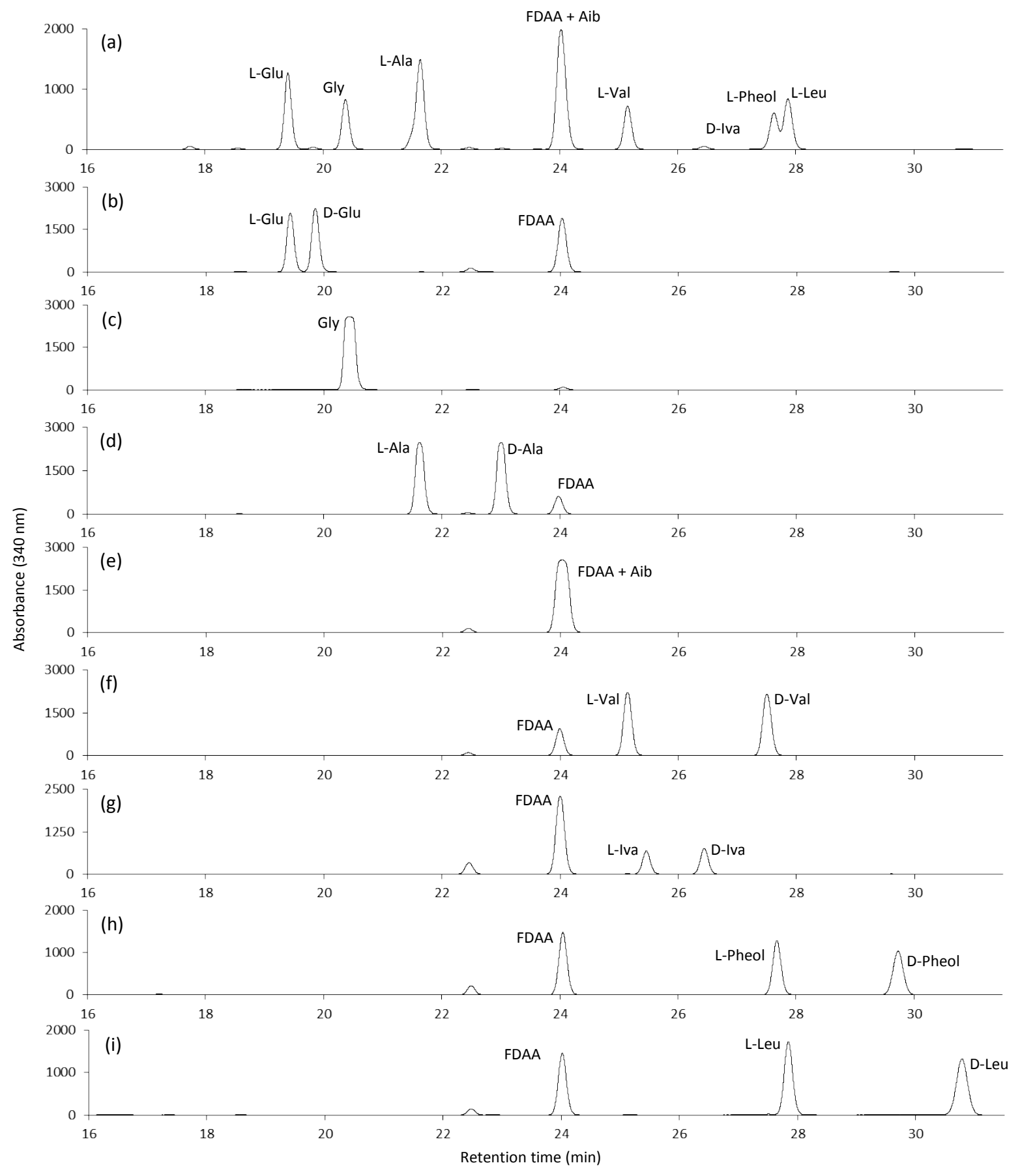


Fig. S59. Marfey's analysis of hydrolyzed pentadecaibin IV (4) derivatized with L-FDAA (a) and comparison with L-FDAA derivatives of standard amino acids (b-h).

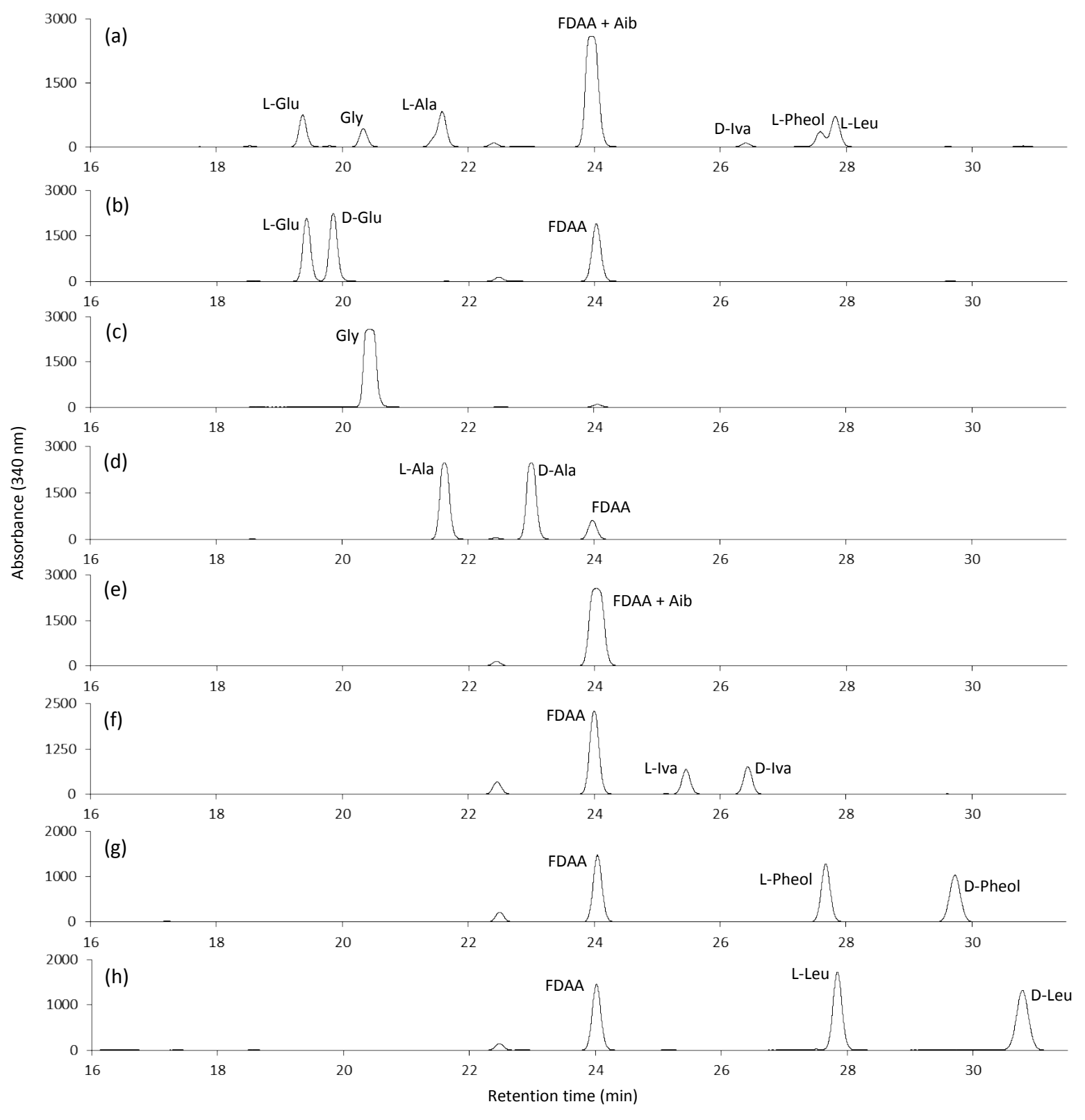


Fig. S60. Marfey's analysis of hydrolyzed pentadecaibin V (5) derivatized with L-FDAA (a) and comparison with L-FDAA derivatives of standard amino acids (b-i).

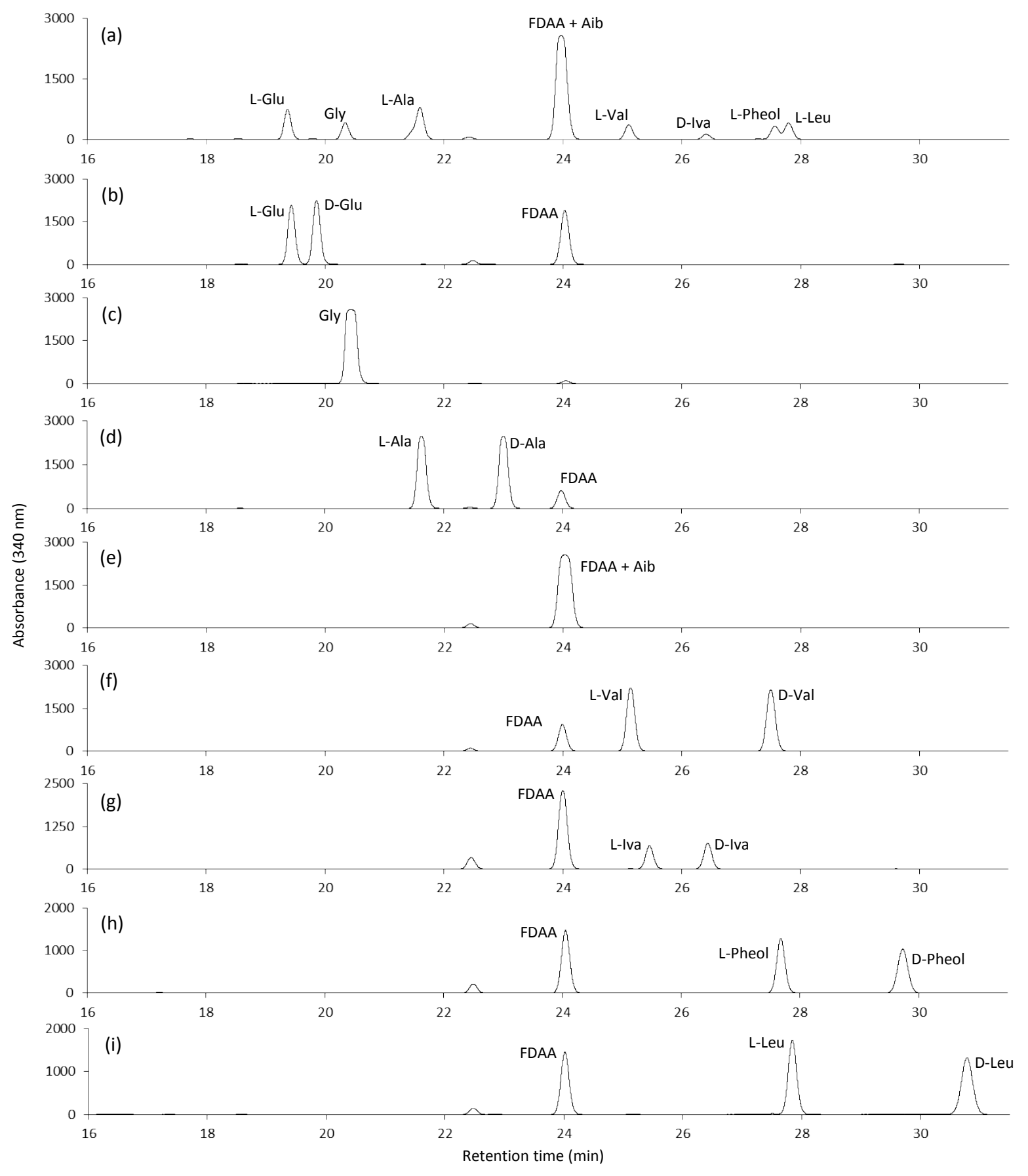


Fig S61. Sequence alignments of pentadecaibins from Trichoderma sp. MMS1255 and known 18-residue peptaibols trichorzins. Sequences are given in standard single-letter code (Ac, acetyl-, U, Aib and ol represents the C-terminal amino-alcohol).

\begin{tabular}{|ccccccccccc}
\hline 1 & 2 & 3 & 4 & 5 & 6 & 7 & 8 & 9 & 10 & 11 \\
AC-U & $G$ & $A$ & $L$ & $U$ & $Q$ & $U$ & $V$ & $U$ & $A$ & $U$ \\
& & & & $J$ & & $J$ & L & & &
\end{tabular}

\begin{tabular}{|cccccccccccccccccc|}
\hline 1 & 2 & 3 & 4 & 5 & 6 & 7 & 8 & 9 & 10 & 11 & 12 & 13 & 14 & 15 & 16 & 17 & 18 \\
AC-U & S & A & U & J & Q & U & V & U & G & L & U & P & L & U & U & Q & Fol \\
& & & J & & & J & & & & & & & & & & &
\end{tabular}

